\title{
WestVirginiaUniversity
}

THE RESEARCH REPOSITORY @ WVU

Graduate Theses, Dissertations, and Problem Reports

2011

\section{Studies of pulsar populations: Searching, timing, and modeling}

Jason Boyles

West Virginia University

Follow this and additional works at: https://researchrepository.wvu.edu/etd

\section{Recommended Citation}

Boyles, Jason, "Studies of pulsar populations: Searching, timing, and modeling" (2011). Graduate Theses, Dissertations, and Problem Reports. 3480.

https://researchrepository.wvu.edu/etd/3480

This Dissertation is protected by copyright and/or related rights. It has been brought to you by the The Research Repository @ WVU with permission from the rights-holder(s). You are free to use this Dissertation in any way that is permitted by the copyright and related rights legislation that applies to your use. For other uses you must obtain permission from the rights-holder(s) directly, unless additional rights are indicated by a Creative Commons license in the record and/ or on the work itself. This Dissertation has been accepted for inclusion in WVU Graduate Theses, Dissertations, and Problem Reports collection by an authorized administrator of The Research Repository @ WVU.

For more information, please contact researchrepository@mail.wvu.edu. 
STUDIES OF PULSAR POPULATIONS:

SEARCHING, TIMING, AND MODELING

\author{
by \\ Jason Boyles \\ Dissertation submitted to \\ the Eberly College of \\ Arts and Sciences at \\ West Virginia University \\ in partial fulfillment of \\ the requirements for \\ the degree of
}

Doctor of Philosophy

in

Physics

Dr. Duncan Lorimer, Ph.D., Chair

Dr. Maura McLaughlin, Ph.D.

Dr. James Lewis, Ph.D.

Dr. Daniel Pisano, Ph.D.

Dr. Tom Wilson, Ph.D.

Department of Physics

Morgantown, West Virginia

2011 


\section{ABSTRACT \\ Studies of Pulsar Populations: \\ Searching, Timing, and Modeling}

Jason Boyles

Pulsars are interesting astronomical objects in their own right but, they are also great instruments to use for physics. Their clock-like periodicity allows them to be used to study the interstellar medium, detect gravitational waves, and study binary evolution. In this work we take a look at a general view of the pulsar population through searching for new pulsars, measuring the properties of pulsars through a technique called pulsar timing, and modeling of the pulsar population using Bayesian techniques.

We took advantage of the immobilization of the Robert C. Byrd Green Bank Telescope during the track refurbishing in 2007 to search the sky for radio pulsars at a frequency of $350 \mathrm{MHz}$. This survey has excellent sensitivity off the Galactic plane but suffers from scattering and dispersion measure smearing in the Galactic plane. So far in this survey we have found 27 new pulsars including six millisecond pulsars of which some are interesting sources that have been studied across the electro-magnetic spectrum.

Rotating radio transients have emission that is periodic but is not always apparent using Fourier domain techniques. These sources may represent a new part of the neutron stars spectrum but present difficulties in studying them. Here we discuss some properties of five rotating radio transients and offer some comments on how they relate to normal radio pulsars.

Globular clusters host a large number of millisecond pulsars. This is expected due to the stellar evolution of the globular cluster. They have a few pulsars much younger than the globular cluster itself. We examined this population of younger pulsars, putting constraints on the possible total population, and examined the globular clusters for evidence of how they could be formed without massive progenitors that are needed for core collapse supernovae. The most likely scenario is accretion induced collapse of a O-Ne-Mg white dwarf. 


\section{Acknowledgments}

This journey could not have been completed alone, and thus many people deserve my thanks and gratitude for their help along the way.

I would like to first start off with my committee chair Dr. Duncan Lorimer. Without his knowledge, guidance, and encouragement this journey may have not taken place.

Next I would like to thank my co-adviser Dr. Maura McLaughlin. Though our points of view differed sometimes, she always helped and kept my best interests in mind.

Next I would like to acknowledge my remaining committee members, Dr. D. J. Pisano, Dr. James Lewis, and Dr. Thomas Wilson, and commend them on enduring what seemed to be the longest oral examination in history.

Of course none of this would be possible without the Physics department of West Virginia University and all of its dedicated employees. Our department chair Dr. Earl Scime gave me the opportunity to come and work here and worked to improve and expand the department especially to add astronomy research to the department. The office staff: Sherry, Siobhan, Devon, Sandy, Amy, and Lori, who all are very helpful and enjoyable people to work with.

I would also like to thank the late Dr. Jack Littleton. Without his many years of dedication and work within the University involving astronomy, the department may have never seen the days where astrophysics is a major research area here at WVU. 
Many collaborators have helped and influenced my work, and deserve to be mentioned. This is a long list but I would personally like to thank our former post-doc Dr. Vlad Kondratiev, for his many hours of assistance at the beginning of my career; Dr. Joris Verbiest, for his many interesting and useful hours of pulsar and non-pulsar related discussion; and Dr. Scott Ransom for his software packages and hands-on help learning how to use his software and operating the Green Bank Ultimate Pulsar Processing Instrument (GUPPI).

I have spent many hours in my windowless office of Hodges 111 and would like to thank Josh R., Josh M., Mitch, Ben, and Priya for their immediate availability to help me when I knew they had the knowledge needed and I did not, and for their conversation when my mind would wander.

Apart from the many hours in Hodges 111, some were spent playing team trivia on Fridays nights and these people helped me relax and realize that there is more to life then just sitting at a computer. Thanks Josh, Steph, Will, Kelly, Sam, Evan, Matt, and Peter for our many accomplishments we obtained in the pursuit of using our knowledge for fun.

Last but not least, I would like to thank my loving Mother, Kathryn Boyles. Without her years of working crappy shifts, putting a roof over my head, and food on the table none of this other thanks would be needed. Thanks Mom. 


\section{Table of Contents}

List of Tables vii

List of Figures $\quad$ ix

List of Abbreviations and Symbols xii

1 Introduction 1

1.1 Pulsar Basics . . . . . . . . . . . . . . . . . . . . . . 1

1.2 Propagation Effects . . . . . . . . . . . . . . . . . 5

1.3 Types of Pulsars . . . . . . . . . . . . . . . . . . . . . . 10

1.3.1 Normal pulsars . . . . . . . . . . . . . . . . . . . 10

1.3.2 Millisecond Pulsars . . . . . . . . . . . . . . . . . . 12

1.3.3 Rotating Radio Transients . . . . . . . . . . . . . . . . . . . . 13

1.4 Pulsar Searching . . . . . . . . . . . . . . . . . . . . . 14

1.4.1 De-dispersion . . . . . . . . . . . . . . . . . 14

1.4.2 Frequency Domain Searches . . . . . . . . . . . . . . . 15

1.4.3 Time Domain Searches . . . . . . . . . . . . . . . 16

1.4.4 Acceleration Searches . . . . . . . . . . . . . . . . . . 17

1.4.5 Radio Frequency Interference . . . . . . . . . . . . . . . . . 18

1.4.6 Candidate Selection . . . . . . . . . . . . . . . . . . 19

1.5 Pulsar Timing . . . . . . . . . . . . . . . . . . . . . . . . . . . . . . . . . 20

1.6 Outline of Thesis . . . . . . . . . . . . . . . . . . . 27

2 A 350-MHz Drift-scan Pulsar Survey with the GBT. 36

2.1 Introduction . . . . . . . . . . . . . . . 36

2.2 Survey Observations . . . . . . . . . . . . . . . . . . . . 38

2.3 Discovery Analysis . . . . . . . . . . . . . . . . . . . . 47

2.4 Timing Observations and Analysis . . . . . . . . . . . . . . . 52

2.5 Other measured parameters . . . . . . . . . . . . . . . . 57

2.5.1 820-MHz Fluxes . . . . . . . . . . . . . . . . . . . 57

2.5.2 Rotation Measures . . . . . . . . . . . . . . . . 58

2.5.3 Pulse Widths at $820 \mathrm{MHz}$. . . . . . . . . . . . . 58

2.6 Discussion . . . . . . . . . . . . . . . . . . . . . 59

2.6 .1 PSR J1327-0755 . . . . . . . . . . . . . . . . 61

2.6.2 PSR J1623-0841 . . . . . . . . . . . . . . . . 61

2.6 .3 PSR J1737-0814 . . . . . . . . . . . . . . . . . . . . 62

2.6 .4 PSR J1941+0121 . . . . . . . . . . . . . . . . . . . . . . . . . 63

2.6 .5 PSR J2222-0137 . . . . . . . . . . . . . . . . 63

2.7 Conclusions and Future Work . . . . . . . . . . . . . . . . 66 
$\begin{array}{lll}3 & \text { Timing Observation of RRATs } & 77\end{array}$

3.1 Introduction . . . . . . . . . . . . . . . . . 77

3.2 Timing and Analysis Techniques . . . . . . . . . . . . . . 78

3.2 .1 TOAs . . . . . . . . . . . . . . . . . . 78

3.2.2 Pulse Profiles and Widths . . . . . . . . . . . . . . . 80

3.3 Timing Results . . . . . . . . . . . . . . . . . . . . . . . 82

3.3 .1 PSR J1623-0841 . . . . . . . . . . . . . . . . 82

$3.3 .2 \quad$ PSR J1739-2521 . . . . . . . . . . . . . . . . . . . . . . 85

3.3 .3 PSR J1754-3014 . . . . . . . . . . . . . . . . . 85

3.3 .4 PSR J1839-0141 . . . . . . . . . . . . . . . . . . . . . . . . . . . . . . . . . 86

3.3 .5 PSR J1848-1243 . . . . . . . . . . . . . . . . 88

3.4 Summary and Further Work . . . . . . . . . . . . . . . . 88

4 Young Radio Pulsars in Galactic Globular Clusters 96

4.1 Introduction . . . . . . . . . . . . . . . . . 96

4.2 The current sample of young pulsars in GCs . . . . . . . . . . . . . 98

4.3 Sensitivity limits for young pulsars in GCs . . . . . . . . . . . . . . 101

4.4 The potentially observable population of young pulsars in GCs . . . . 102

4.5 The intrinsic population and birth rates of young GC pulsars . . . . . 106

4.5.1 Results for all GCs . . . . . . . . . . . . . . . . 106

4.5.2 Metal-rich GCs . . . . . . . . . . . . . . . . . . . 108

4.6 Discussion . . . . . . . . . . . . . . . . . . . . . . 110

4.6.1 Flux Luminosity Limits . . . . . . . . . . . . . . . . 110

4.6.2 Birth Rates in GCs . . . . . . . . . . . . . . . . . . . . . . . 110

4.6.3 Role of cluster metallicity . . . . . . . . . . . . . . . . 111

4.6.4 Formation scenarios . . . . . . . . . . . . . . . . . . . 112

4.6.4.1 Blue Stragglers . . . . . . . . . . . . . . . . 112

4.6.4.2 Electron Capture Supernovae . . . . . . . . . . . . . 113

4.6.4.3 Galactic Bulge Pulsars . . . . . . . . . . . . . . . 116

4.6.5 Suggested Future Work . . . . . . . . . . . . . . . . . . . 119

5 Conclusions and Future Work 130

5.1 Green Bank 350-MHz surveys . . . . . . . . . . . . . . . . . . . . 130

5.2 Rotating Radio Transients . . . . . . . . . . . . . . . . . . . . . 132

5.3 Globular Cluster Pulsars . . . . . . . . . . . . . . . . . . . . . 133

5.4 Next Generation Radio Telescopes . . . . . . . . . . . . . . . . . . 133 


\section{List of Tables}

1.1 Parameter file for PSR J1939+2134 created using the ATNF pulsar catalog. For a listing of each parameter's definition see the TEMPO website (http://tempo.sourceforge.net). . . . . . . . . . . . . 22

1.2 An example TEMPO TOA files for PSR J2111+2108 showing one year's worth of TOAs. The TOAs are in Princeton format. The one digit site code is a character assigned to each observatory with 1 representing the GBT, frequency is the center frequency of the observation, TOA is the measured time when the pulse is recorded, error is the measured error on the TOA, and DM is the dispersion measure of the pulsar when it was observed. . . . . . . . . . . . 23

2.1 Observations . . . . . . . . . . . . . . . . . . . . . . . . 41

2.1 cont. . . . . . . . . . . . . . . . . . . 42

2.1 cont. . . . . . . . . . . . . . . . . . . . 43

2.1 cont. . . . . . . . . . . . . . . . . . . . . 44

2.1 cont. . . . . . . . . . . . . . . . . . . 45

2.1 cont. . . . . . . . . . . . . . . . . . . 46

2.1 Start MJD, scan length, azimuth, elevation, and number of data bits for each observation. . . . . . . . . . . . . . . . 4 46

2.2 Parameters for DMs searched. Each de-dispersed time series was processed using 128 subbands. . . . . . . . . . . . . . . . . . . . 47

2.3 Post-gridding best-fit positions of pulsars. $\Delta \mathrm{P}$ is the difference between the true position and the discovery and post-gridding positions. 53

2.4 Observational parameters for timing observations. . . . . . . . . 55

2.5 RVM model fit results for PSR J1941+0121. Here $\phi_{0}$ is the pulse phase of magnetic meridian, $\psi_{0}$ is position angle at $\phi_{0}, \alpha$ is the colatitude of magnetic axis, and $\zeta$ is the colatitude of the observer's line of sight. . . . . . . . . . . . . . . . . . 6 64

2.6 Timing and derived parameters for newly discovered isolated pulsars. 68

2.7 Timing and derived parameters for newly discovered isolated pulsars. $\quad 69$

2.8 Timing and derived parameters for newly discovered isolated pulsars. $\quad 70$

2.9 Timing and derived parameters for newly discovered isolated pulsars. 71

2.10 Timing and derived parameters for newly discovered isolated pulsars. 72

2.11 Timing and derived parameters for newly discovered binary pulsars. 73

2.12 Timing and derived parameters for newly discovered PSR J2222-0137. 74

3.1 Table containing all observations of PSR 1839-0141. Integer MJDs have no detection of PSR 1839-0141 in either periodicity searches or single-pulse searches. 
3.2 Table containing the discovery position and final position for five RRATs. PSR J1739-2521 and PSR J1839-0141 were both discovered outside the FWHM of the telescope beam size of their survey. PSR J1623-0841 was discovered at $350 \mathrm{MHz}$ with the GBT and its beam is $\sim 36^{\prime}$. . . . . . . . . . . . . . . . . . . . . . . . . 90 90

3.3 Fitted and derived parameters for RRATs. . . . . . . . . . . . . . 91

3.4 Fitted and derived parameters for RRATs. . . . . . . . . . . . . 92

3.5 Fitted and derived parameters for RRATs. . . . . . . . . . . . . . . 93

4.1 Properties of the four young pulsars currently known in GCs. . . . . . 100

4.2 Parameters for Bulge Globular Clusters. $\mathrm{D}_{\mathrm{GC}}$ is the distance from the Galactic center and D is the distance from the Sun to the Globular cluster. GC parameters from Harris (1996) . . . . . . . . . . . . . 116

4.3 Parameters for globular clusters searched for pulsars. GC parameters from Harris (1996). . . . . . . . . . . . . . . . . . 126

4.3 Continued . . . . . . . . . . . . . . . . . . . . . 127

4.4 Binomial analysis of young pulsars in globular clusters. . . . . . . . 128

4.4 Continued . . . . . . . . . . . . . . . . . . . . 129 


\section{List of Figures}

1.1 Frequency dependent time delay in the pulse arrival due to ISM effects. The sloped vertical black lines are the pulses from PSR B1642-03, which has a spin period of $388 \mathrm{~ms}$ and a dispersion measure of $35.7 \mathrm{pc} \mathrm{cm}^{-3}$. Parts of three pulses are shown. . . . . . . . . . 5

1.2 Profile of $431 \mathrm{~ms}$ PSR B1900-06 at a DM of $195 \mathrm{pc} \mathrm{cm}^{-3}$ showing the "1/e" scattering tail induced by the pulses traveling through the ISM. Dispersion Measure of the pulsar is $195 \mathrm{pc} \mathrm{cm}^{-3}$. . . . . . . . . 7

1.3 The measured distribution of $P$ and $\dot{P}$ for all known pulsars. Lines of constant characteristic age (dot-dash), constant magnetic field (dash), and constant spindown luminosity (dot) are shown. Data are taken from the ATNF pulsar catalog (Manchester et al. 2005). . . . . . . .

1.4 Top: Time series of a fake pulsar created with a period of $0.975 \mathrm{~s}$ and a pulse width of $24 \%$. The individual pulses can not be seen. Bottom: FFT of the time series with only the first 2000 FFT bins shown for clarity. The first and second harmonics are clearly visible.

1.5 Single pulse search output of PSR J1754-30. The top middle and top right plots show excess number of detections at the DM of the pulsar while the bottom plot shows detections as a function of time and DM with circle size being proportional to signal-to-noise of the pulse. In this observation, about a dozen pulses can be seen centered around a DM of $99 \mathrm{pc} \mathrm{cm}^{-3} \ldots$. . . . . . . . . . . . . . . .

1.6 A set of diagnostic plots for an observation of PSR J2221-01. The left hand side shows a pulse profile at the top and a plot of intensity versus time and pulse phase on the bottom. Along the plot of intensity versus time is a plot of $\chi^{2}$ versus time. The middle top plot shows frequency versus pulse phase and the bottom shows $\chi^{2}$ versus DM. The right hand side (from top to bottom) shows $\chi^{2}$ versus period, $\chi^{2}$ versus $\dot{P}$, and an intensity plot of period- $\dot{P}$ phase space. . . . . . . 30

1.7 An example of a weak redetected pulsar in the $350-\mathrm{MHz}$ drift-scan survey. PSR B1829-08 has a period of $647 \mathrm{~ms}$, a DM of $301 \mathrm{pc}$ $\mathrm{cm}^{-3}$, and a 400-MHz flux of $9.9 \mathrm{mJy}$. Pulse scattering has reduced the pulsar's signal to almost an undetectable level. . . . . . . . . . . . 31

1.8 Example of pure noise showing none of the characteristics of a detected pulsar. . . . . . . . . . . . . . . . . . 32

1.9 A diagnostic plot showing a $1.2 \mathrm{~s}$ RFI signal. Besides the plot of significant versus DM, this plot shows all the characteristics of a pulsar. 
1.10 Timing residuals for PSR B1133+16. (a) Shows a timing model with no systematics and a random distribution of TOAs, indicating a good model fit. (b) Timing model showing the parabolic signature of underestimating $\dot{P}$ by $4 \%$. (c) Timing model showing the constant amplitude yearly sinusoidal signature of a offset in position (here 1 arcmin in declination). (d) Timing model showing the linearly increasing amplitude, yearly sinusoidal signature of not including proper motion in the timing model. Figure used with permission from the Handbook of Pulsar Astronomy (Lorimer \& Kramer 2005). . . . . .

1.11 A sinusoidal fit to the barycentered data for PSR J2222-0137 with an orbital period of 2.45 days and a semi-major axis of 10.8 lt-s. The fit is slightly imperfect due to the pulsar's non-zero orbital eccentricity. 35

2.1 A map in equatorial coordinates showing the sky coverage of the survey. The drift-scan portion is shown in black, the PSC portion is shown in gray, and the red line shows the Galactic plane. New discoveries presented in this work are shown as red stars. . . . . . . . 39

2.2 Single-pulse search output showing the discovery observation of newly discovered pulsar PSR J2111+2108. . . . . . . . . . . . . . . 50

2.3 Example of the search output diagnostic plot for the newly discovered pulsar PSR J2222-0137. . . . . . . . . . . . . . . . . . 51

2.4 Example fit for RM for PSR J1941+0121. Square boxes are the measured values while the dotted line is the best fit value.

$2.5 P-\dot{P}$ diagram showing the new discoveries as green diamonds. The pulsar population is taken for the ATNF pulsar catalog (Manchester et al. 2005). . . . . . . . . . . . . . . . . . . . . .

2.6 RVM model fit for the radio emission of PSR J1941+0121. The top plot show parallactic angle versus pulse phase, while the bottom plot shows integrated pulse flux density versus pulse phase for total intensity (black line), linearly polarized flux (red line), and circularly polarized flux (blue line). . . . . . . . . . . . . . . . . . . . . . 64

2.7 TEMPO2 simulations showing the Shapiro delay expected for PSR J2222-0137 for multiple inclination angles. The $\mathrm{x}$-axis is the orbital phase of the pulsar with respect to periastron and the y-axis is the excess time delay caused by the companion as the pulsar's signal passes through the warped space-time around the companion. From top to bottom: $\mathrm{i}=90^{\circ}$ (red), $\mathrm{i}=80^{\circ}$, (green), and $\mathrm{i}=70^{\circ}$ (blue).

2.8 Profiles at $820 \mathrm{MHz}$ for nine newly discovered pulsars created from multi-epoch data.

2.9 Profiles at $820 \mathrm{MHz}$ for four newly discovered pulsars created from multi-epoch data. . . . . . . . . . . . . . . . . . . .

3.1 A single observation plot for PSR J1848-12 showing TOAs varying by more than the error bars on short timescales, possibly indicating that pulse jittering is occurring. 
3.2 A $P-\dot{P}$ diagram showing the new RRATs as black diamonds and previously timed RRATs as blue squares. The pulsar population is taken from the ATNF pulsar catalog (Manchester et al. 2005)

3.3 Two single-pulse detections for PSR J1623-0841 at two different pointings taken at $350 \mathrm{MHz}$ with the GBT. The top is offset by $20^{\prime}$ and the bottom is on source. . . . . . . . . . . . . . . . 83

3.4 Three separate observations of PSR J1739-2521 showing the intermittent nature of this source. . . . . . . . . . . . . . . 84

3.5 A single-pulse plot of PSR J1848-1243 (top) and a folded detection (bottom) shown. . . . . . . . . . . . . . . . . . . . 89

3.6 Pulse profiles for PSR J1625-0841 and PSR J1754-3014 at $350 \mathrm{MHz}$ and $820 \mathrm{MHz}$. The profiles are created by folding each observation and summing all profiles together. . . . . . . . . . . . . . . . . . . . 94

3.7 Pulse profile at $820 \mathrm{MHz}$ for the rest for the rest of the RRATs. Both of PSR J1739-2521 and PSR J1839-0141 profiles are a sum of data only when the pulsar is on. PSR J1848-1243's profile is created in the same way as for PSR J1625-0841 and PSR J1754-3014 . . . . . 95

$4.1 P-\dot{P}$ diagram showing young globular cluster pulsars as black stars and PSR J1750-37A as a red square. PSR B2127+11A is shown as a blue arrow with the arrow representing the limit on period derivative at the bottom of the diagram. The pulsar population data are taken from the ATNF pulsar database (Manchester et al 2005). . . . . . . 100

4.2 Examples of discrete posterior probability density functions for the number of potentially observable pulsars in GCs using the Bayesian analysis. The number of known young pulsars are given in the upper left corner under the GC's name in each plot. . . . . . . . . . . . . . 105

4.3 Survey luminosity limits at $1400 \mathrm{MHz}$ as a function of metallicity with six GCs excluded with distances greater then $20 \mathrm{kpc}$. The luminosities are randomly distributed and show no bias against low or high metallicity GCs. . . . . . . . . . . . . . . . . . . . . . . . . . 122

4.4 CDF of young pulsars predicted by the binomial analysis as a function of metallicity. . . . . . . . . . . . . . . . . . . . . 123

4.5 CDF of birth rate versus metallicity for multiple velocity dispersions. The solid black line represents an estimate of ECS rates in GCs. . . . 124

4.6 Histogram of number of predicted observable young pulsars per GC using a model only dependent on the mass of the GC. . . . . . . . . . 125 


\section{List of Abbreviations and Symbols}

$B$

$b$

$\mathrm{c}$

$\mathrm{CDF}$

DFT

$\mathrm{DM}$

e

$\dot{E}$

EoS

$f$

FFT

$\mathrm{GC}$

$\mathrm{GHz}$

GUPPI $i$

I

ISM

Jy

$\mathrm{kpc}$

$L$

$l$

lt-s

$\mathrm{M}[$ number]

$M_{\odot}$

$m_{\mathrm{e}}$

$\mathrm{MHz}$

Myr

MJD

MSP
Angle Between Rotational and Magnetic Field Axes

Angle Between Magnetic Axis and Line of Sight Trajectory

Pulse Duty Cycle

Angular Frequency

Frequency

Frequency Derivative

Characteristic Age

Scattering Time

Magnetic Field

Galactic Latitude

Speed of Light

Cumulative Distribution Function

Discrete Fourier Transform

Dispersion Measure

Charge of an Electron

Spin-down Luminosity

Equation of State

Observing Frequency

Fast Fourier Transform

Globular Cluster

Gigahertz

Green Bank Ultimate Pulsar Processing Instrument

Orbital Inclination Angle

Moment of Inertia

Interstellar Medium

Jansky

Kiloparsec

Luminosity

Galactic Longitude

Light Second

Messier Object [number]

Mass of the Sun

Mass of an Electron

Megahertz

Megayear

Modified Julian Day

Millisecond Pulsar 


$\begin{array}{ll}n & \text { Braking Index } \\ n_{\mathrm{e}} & \text { Electron Density } \\ \text { NGC } & \text { New General Catalog } \\ \text { NS } & \text { Neutron Star } \\ P & \text { Rotational Period } \\ \dot{P} & \text { Rotational Period Derivative } \\ \text { PMPS } & \text { Parkes Multibeam Pulsar Survey } \\ \text { PSR } & \text { Pulsar } \\ \text { PDF } & \text { Probability Density Function } \\ R & \text { Radius of a Neutron Star } \\ \text { RRAT } & \text { Rotating Radio Transient } \\ \text { RFI } & \text { Radio Frequency Interference } \\ \text { S } & \text { second } \\ S & \text { Flux Density } \\ \text { S/N } & \text { Signal-to-Noise Ratio } \\ \text { WD } & \text { White Dwarf }\end{array}$




\section{Chapter 1}

\section{Introduction}

Pulsars are rapidly rotating, highly magnetized neutron stars whose radio emission we see as pulses as their radio beams cross our line of sight. In the following sections, the details of pulsars will be discussed and a general introduction will be provided. We pay particular attention to those properties which are the most relevant for this dissertation. For further details, see the Handbook of Pulsar Astronomy (Lorimer \& Kramer 2005) and references therein.

\subsection{Pulsar Basics}

Pulsars are highly compact astrophysical objects. It was proposed by Baade \& Zwicky (1934) that neutron stars might form during the collapse of massive stars (e.g. in supernovae). The discovery of the first pulsar (CP 1919; Hewish et al. 1968) provided the first evidence that neutron stars may exist. Further theoretical work provided solid proof that only neutron stars could match the observed parameters seen in pulsar observations (Gold 1969).

The exact structure and equation of state (EoS) of neutron stars are not well understood, but observational evidence has proved some clues. The crust is a crystallized solid, probably made of iron, with a super-fluid, superconducting core. This has been deduced from glitches, sudden increases in spin period, in pulsars, most 
noticeably the Vela pulsar (Anderson \& Itoh 1975). The exact composition of the core is unknown but most models suggest a sea of mostly neutrons with some more exotic models suggestioning that the inner most core could be strange quark matter (Kurkela et al. 2010). Pulsars are the most dense objects in the universe outside of stellar mass black holes. EoSs predict masses $(M)$ in the range $1.0-3.0 M_{\odot}$ and radii $(R)$ of $10-15 \mathrm{~km}$, with $M=1.4 M_{\odot}$ and $R=10 \mathrm{~km}$ taken as the canonical values for each parameter (Lattimer \& Prakash 2001). The average density for a canonical pulsar $\left(6.7 \times 10^{14} \mathrm{~g} \mathrm{~cm}^{-3}\right)$ is slightly in excess of nuclear matter $(2.7$ $\left.\times 10^{14} \mathrm{~g} \mathrm{~cm}^{-3}\right)$. Assuming a uniform spherical distribution of mass for a pulsar, a canonical value of $10^{45} \mathrm{~g} \mathrm{~cm}^{2}$ is obtained for the moment of inertia $(I)$.

The rotational period $(P)$ for pulsars ranges from 1.4 ms for PSR J1748-2446ad (Hessels et al. 2006) to 8.5 s for PSR J2144-3933 (Manchester et al. 1996). Pulsar periods are not constant over their lifetimes and are observed increase with time, i.e. they exhibit a rotational period derivative $(\dot{P})$. The distribution in $P$ and $\dot{P}$ is bimodal, separating pulsars into two main categories: "normal" pulsars and "millisecond" pulsars. The properties of these two types of pulsars are to follow in section 1.3 .1 and 1.3.2.

The spin-down of a pulsar is the result of the star losing its rotational kinetic energy. Assuming a constant moment of inertia, $I$, this can be represented as:

$$
\dot{E}=\frac{d}{d t} \frac{1}{2} I \Omega^{2}=I \Omega \dot{\Omega}
$$

where $\Omega$ is the angular frequency. This is known as the total spin-down luminosity 
of the pulsar. Inserting the canonical value for $I$ and transforming into the more often used variables of $P$ and $\dot{P}$ we get

$$
\dot{E}=3.95 \times 10^{31} \mathrm{erg} \mathrm{s}^{-1}\left(\frac{\dot{P}}{10^{-15} \mathrm{~s} \mathrm{~s}^{-1}}\right)\left(\frac{P}{\mathrm{~s}}\right)^{-3}
$$

Due to conservation of magnetic flux during the supernova explosion, the surface magnetic fields $\left(B_{\mathrm{S}}\right)$ of pulsars are predicted to range from $10^{8}-10^{14}$ Gauss $(\mathrm{G})$. Though a direct measurement of a pulsar's $B_{\mathrm{S}}$ can not be made, an inferred value can be produced. By assuming all the spin-down energy is dominated by magnetic dipole braking, it can be shown that

$$
B_{\mathrm{S}}=B(r=R)=\sqrt{\frac{3 c^{3} I P \dot{P}}{8 \pi^{2} R^{6} \sin ^{2} \alpha}},
$$

where $\alpha$ is the angle between the magnetic field and spin axes. Setting $I$ and $R$ to their canonical values and choosing the magnetic field and spin axis to be perpendicular $\left(\alpha=90^{\circ}\right)$, we get

$$
B_{\mathrm{S}}=3.2 \times 10^{19} \mathrm{G} \sqrt{P \dot{P}}
$$

Caution should be taken in use of this value because the magnetic field on the surface is not uniform or purely dipolar magnetic field and should be used as only an order-of-magnitude estimate of the magnetic field strength.

Another order-of-magnitude estimate we can make for a pulsar is its characteristic age $\left(\tau_{\mathrm{c}}\right)$. This can be derived from assuming the radiation power is equal to 
the spin-down luminosity and frequency evolution has a power law form of

$$
\dot{\nu}=-\mathrm{K} \nu^{n}
$$

where $\nu$ is the rotational frequency $(1 / P), \dot{\nu}$ is the frequency derivative $\left(-\dot{P} P^{-2}\right), \mathrm{K}$ is a constant, and $n$ is the braking index ( $n=3$ for pure dipole braking). Changing the variables to $P$ and $\dot{P}$ in equation 1.5 and integrating over $P$ we obtain the age of the pulsar

$$
T=\frac{P}{(n-1)}\left[1-\left(\frac{P_{o}}{P}\right)^{n-1}\right]
$$

where $P_{o}$ is the initial period of the pulsar. Assuming pure magnetic dipole radiation and the initial period being much less than the current period, the above equation simplifies down to the characteristic age

$$
\tau_{\mathrm{c}}=\frac{P}{2 \dot{P}}=15.8 \mathrm{Myr}\left(\frac{P}{\mathrm{~s}}\right)\left(\frac{\dot{P}}{10^{-15} \mathrm{~s} \mathrm{~s}^{-1}}\right)^{-1}
$$

Caution needs to be taken when using the $\tau_{\mathrm{c}}$, but unlike the case for $B_{\mathrm{S}}$, we do have measured ages for pulsars. The Crab pulsar (PSR B0531+21) was produced in a supernova in $1054 \mathrm{AD}$ and has a $\tau_{\mathrm{c}}$ of $1240 \mathrm{yrs}$, which are in reasonable agreement. But larger differences have been seen in other pulsars (e. g. PSR J0538+2817; Kramer et al. 2003). 


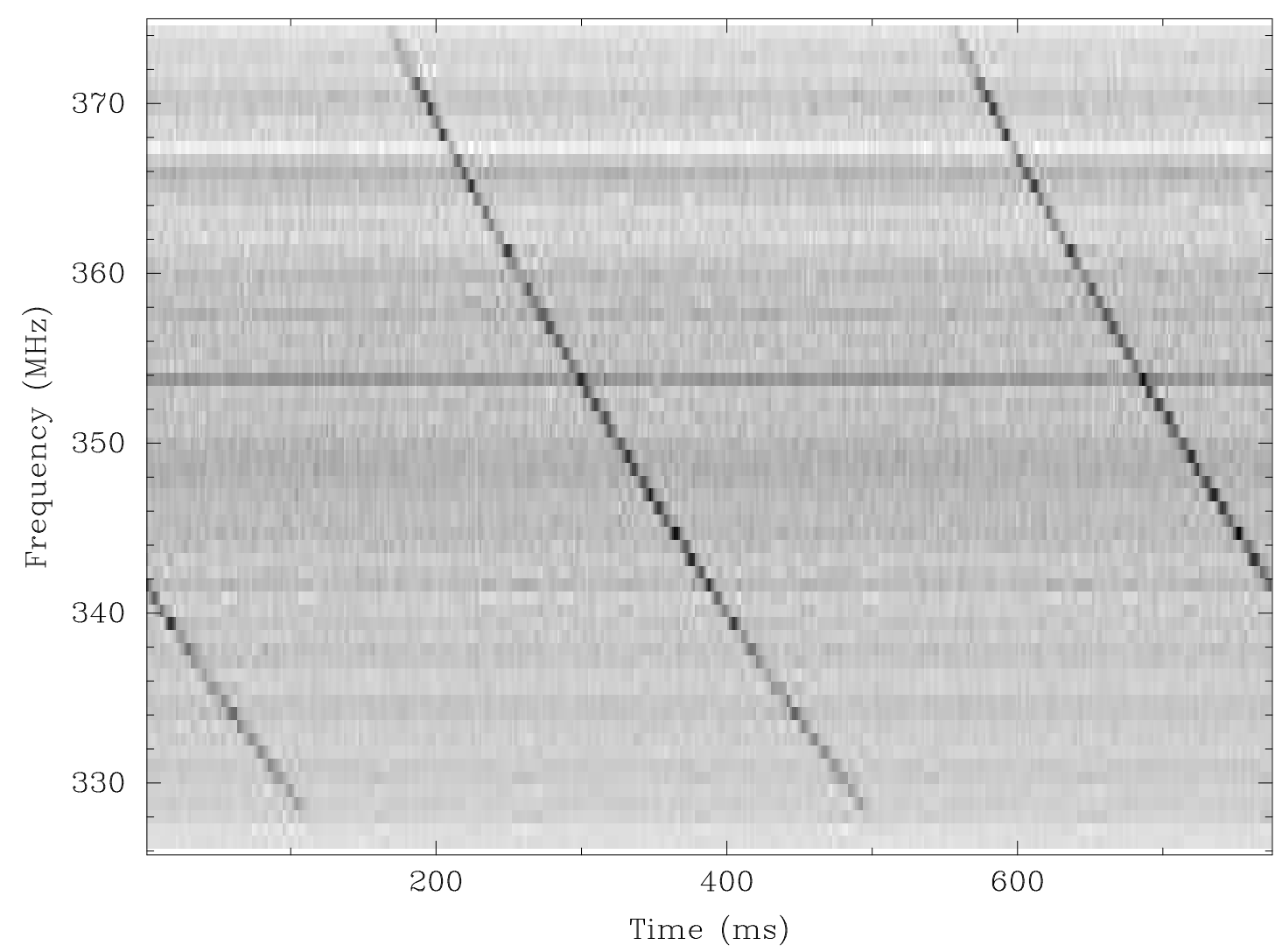

Figure 1.1: Frequency dependent time delay in the pulse arrival due to ISM effects. The sloped vertical black lines are the pulses from PSR B1642-03, which has a spin period of $388 \mathrm{~ms}$ and a dispersion measure of $35.7 \mathrm{pc} \mathrm{cm}^{-3}$. Parts of three pulses are shown.

\subsection{Propagation Effects}

The interstellar medium (ISM) can be modeled as a cold homogeneous plasma characterized by a frequency dependent index of refraction. The dependence causes a time delay that disperses the pulsar's signal. To recover the full flux density of the pulsar, a correction must be applied to the data. The time delays between two frequencies is

$$
\Delta t=4.148808 \times 10^{6} \mathrm{~ms} \times\left(f_{1}^{-2}-f_{2}^{-2}\right) \times \mathrm{DM},
$$


where the two frequencies, $f_{1}$ and $f_{2}$ are measured in $\mathrm{MHz}$ and dispersion measure $(\mathrm{DM})$ is measured in $\mathrm{pc} \mathrm{cm}^{-3}$ and is given by

$$
\mathrm{DM}=\int_{0}^{d} n_{\mathrm{e}} \mathrm{d} l,
$$

where $n_{\mathrm{e}}$ is the electron density along the line of sight in $\mathrm{cm}^{-3}$ and $d$ is the distance to the pulsar in pc. Figure 1.1 shows this propagation effect for an observation with the Green Bank Telescope at a center frequency of $350 \mathrm{MHz}$ and $50 \mathrm{MHz}$ of bandwidth. The DM can be used as an estimate to determine the distance to the pulsar using an electron density model of the Galaxy. The most common one used is the NE2001 electron model by Cordes \& Lazio (2002) which incorporates Galactic spiral-arm structure.

The time delay across the bandwidth also occurs within individual frequency channels and is a result of a continuous signal being quantized due to finite frequency channel widths. This introduces so-called "DM smearing" within an individual frequency channel and is characterized by a smearing time scale

$$
t_{\mathrm{DM}}=8.3 \times 10^{6} \mathrm{~ms} \times \mathrm{DM} \times \Delta f \times f^{-3},
$$

where $\Delta f$ is the width of the frequency channel in $\mathrm{MHz}$ and $f$ is the center frequency of the channel in MHz. When observing pulsars, a careful choice needs to be made to ensure that the DM smearing is not a significant fraction of the pulse period.

Along with DM time delays, the ISM can always cause multi-path propagation of wavefronts, resulting in a scattering tail in pulsar's pulse profiles. This scattering 


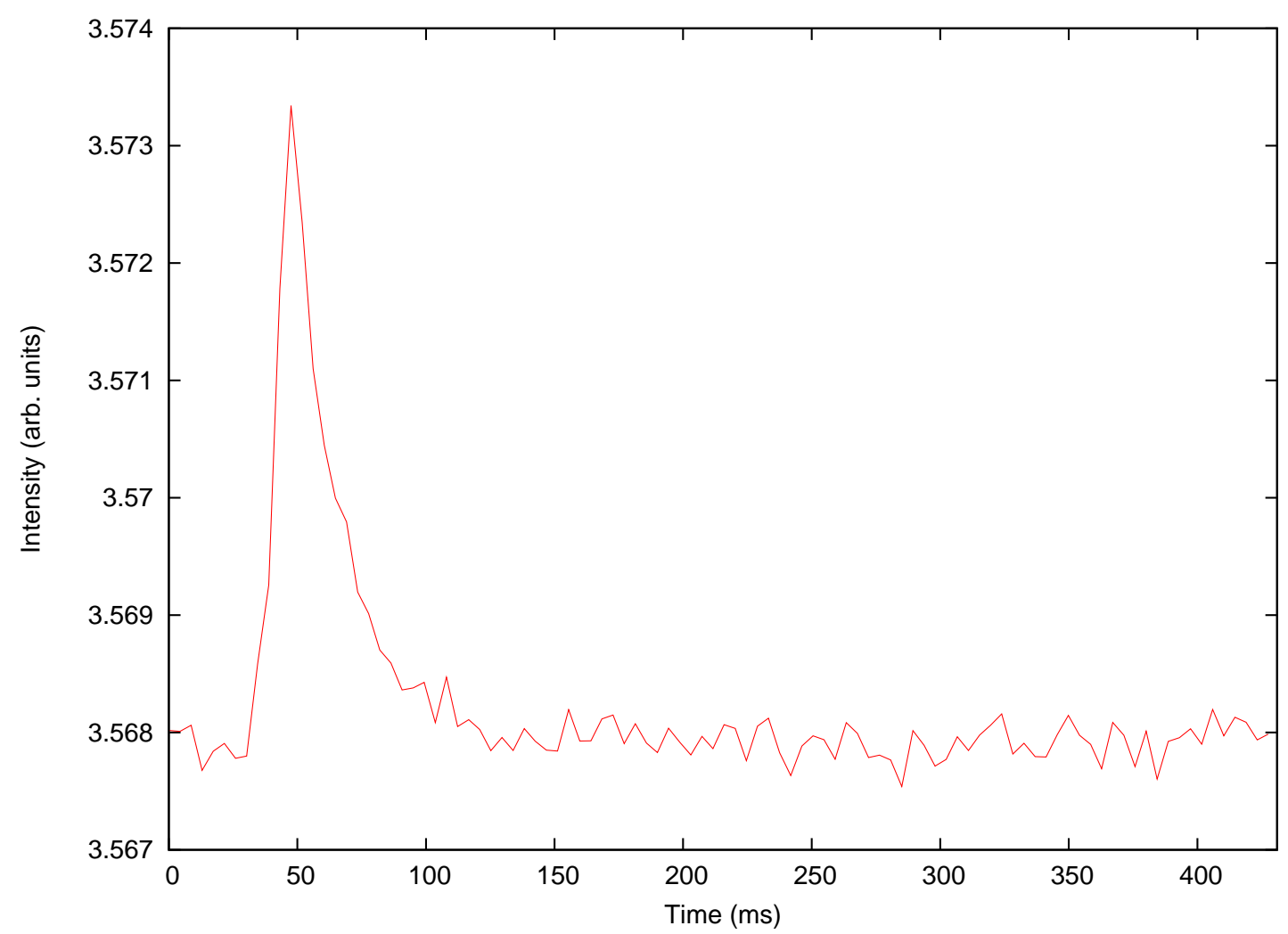

Figure 1.2: Profile of $431 \mathrm{~ms}$ PSR B1900-06 at a DM of $195 \mathrm{pc} \mathrm{cm}^{-3}$ showing the " 1 e" scattering tail induced by the pulses traveling through the ISM. Dispersion Measure of the pulsar is $195 \mathrm{pc} \mathrm{cm}^{-3}$. 
can be characterized by a " $1 / \mathrm{e}$ " tail on the back of the pulse profile with a time constant $\tau_{\text {scat }}$. Figure 1.2 shows a pulsar with this scattering tail. Through this scattering is not uniform, it depends on frequency, DM, and the ISM along the line of sight. An empirical relationship has been derived from real data and has the form

$$
\log _{10}\left(\tau_{\text {scat }}\right)=-6.46+0.154 \log _{10}(\mathrm{DM})+1.07\left(\log _{10} \mathrm{DM}\right)^{2}-3.86 \log _{10}(f)
$$

where $\tau_{\text {scat }}$ is measured in $\mathrm{ms}, \mathrm{DM}$ is measured in $\mathrm{pc} \mathrm{cm}^{-3}$, and $f$ is frequency measured in $\mathrm{GHz}$ (Bhat et al. 2004). This expression can be used to estimate the scattering time for a given observation, though the scatter between this relation and the value measured for observed pulsars is quite large.

The magnetic field of the Galaxy acts as a Faraday screen causing a rotation in the position angle of polarization of the pulsar's signal. Due to pulsars being some of the most polarized astronomical sources, this effect can be seen in many pulsars. This causes a change in the polarization position angle of

$$
\Delta \Psi_{\mathrm{PPA}}=\Delta \Psi_{\text {Faraday }} / 2=\lambda^{2} \times \mathrm{RM}
$$

where $\lambda$ is wavelength and the rotation measure (RM) is defined as

$$
\mathrm{RM}=\frac{e}{2 \pi m_{e}^{2} c^{4}} \int_{0}^{d} n_{\mathrm{e}} B_{\|} \mathrm{d} l,
$$

where $e$ is the charge of the electron, $m_{e}$ is the mass of the electron, $c$ is the speed 
of light, and $B_{\|}$is the average Galactic magnetic field along the line of sight. A measurement of DM and RM will provide the average magnetic field strength along the line of sight, but caution needs to be taken using this measurement. The electron density is not homogeneous and the magnetic field can change direction. These effects may result in incorrect interpretation of the measurement if not treated properly.

Many types of scintillation exist - weak and strong, refractive and diffractive - but one dominates the observations presented in this dissertation, strong diffractive scintillation. Diffractive interstellar scintillation (DISS) manifests itself as strong intensity variations in both time and frequency. DISS is caused by diffraction of electromagnetic radiation as it travels through the ISM, creating constructive and destructive interference patterns analogous to the interference patterns in the double- and single-slit experiment. These patterns have measurable properties that can be used to understand the ISM through which the radiation passed.

Two characteristic quantities can be measured from the dynamic spectrum of a pulsar. The first is a timescale

$$
\Delta t_{\mathrm{DISS}}=\frac{s_{o}}{V_{\mathrm{ISS}}}
$$

where $s_{o}$ is the field coherence scale and $V_{\text {ISS }}$ is the transverse velocity of the pulsar, and the other is a frequency scale,

$$
\Delta f_{\text {DISS }}=\frac{1.16}{2 \pi \tau_{\text {scat }}}
$$


for a Kolmogorov spectrum. With both $\Delta t_{\text {DISS }}$ and $\Delta f_{\text {DISS }}$ measured from a dynamic spectrum, a transverse velocity

$$
V_{\mathrm{ISS}}=A\left(\frac{d}{\mathrm{kpc}}\right)^{1 / 2}\left(\frac{\Delta f_{\mathrm{DISS}}}{\mathrm{MHz}}\right)^{1 / 2}\left(\frac{f}{\mathrm{GHz}}\right)^{-1}\left(\frac{\Delta t_{\mathrm{DISS}}}{\mathrm{s}}\right)^{-1}
$$

can be calculated. Here $d$ is the distance to the pulsar, $f$ is the frequency of the observation, and $A$ is a constant whose value varies depends on the type of screen but has a value in the few thousand kilometers per second range. For most pulsar observations diffractive scintillation is a hindrance but it has been used to help discover pulsars only detectable during DISS maximums when constructive interference increases the pulsar's flux to the point of detection. This technique has been used to detect pulsars in the globular cluster 47 Tucanae (Camilo et al. 2000).

\subsection{Types of Pulsars}

As stated in the previous section, pulsars can be grouped into different classes. In the following section, three types of radio pulsars will be introduced and discussed: normal pulsars, millisecond pulsars (MSPs), and rotating radio transients (RRATs). In each section the properties that make each class distinct will be discussed.

\subsubsection{Normal pulsars}

Normal pulsars are the most common variety of pulsar. In Figure 1.3 they are seen as the large group of pulsars clustered around a period of $1 \mathrm{~s}$ and $\dot{P}$ of $10^{-15} \mathrm{~s} \mathrm{~s}^{-1}$. These pulsars have typical $B$ between $10^{11}$ and $10^{13} \mathrm{G}$ and $\tau_{\mathrm{c}}$ between 


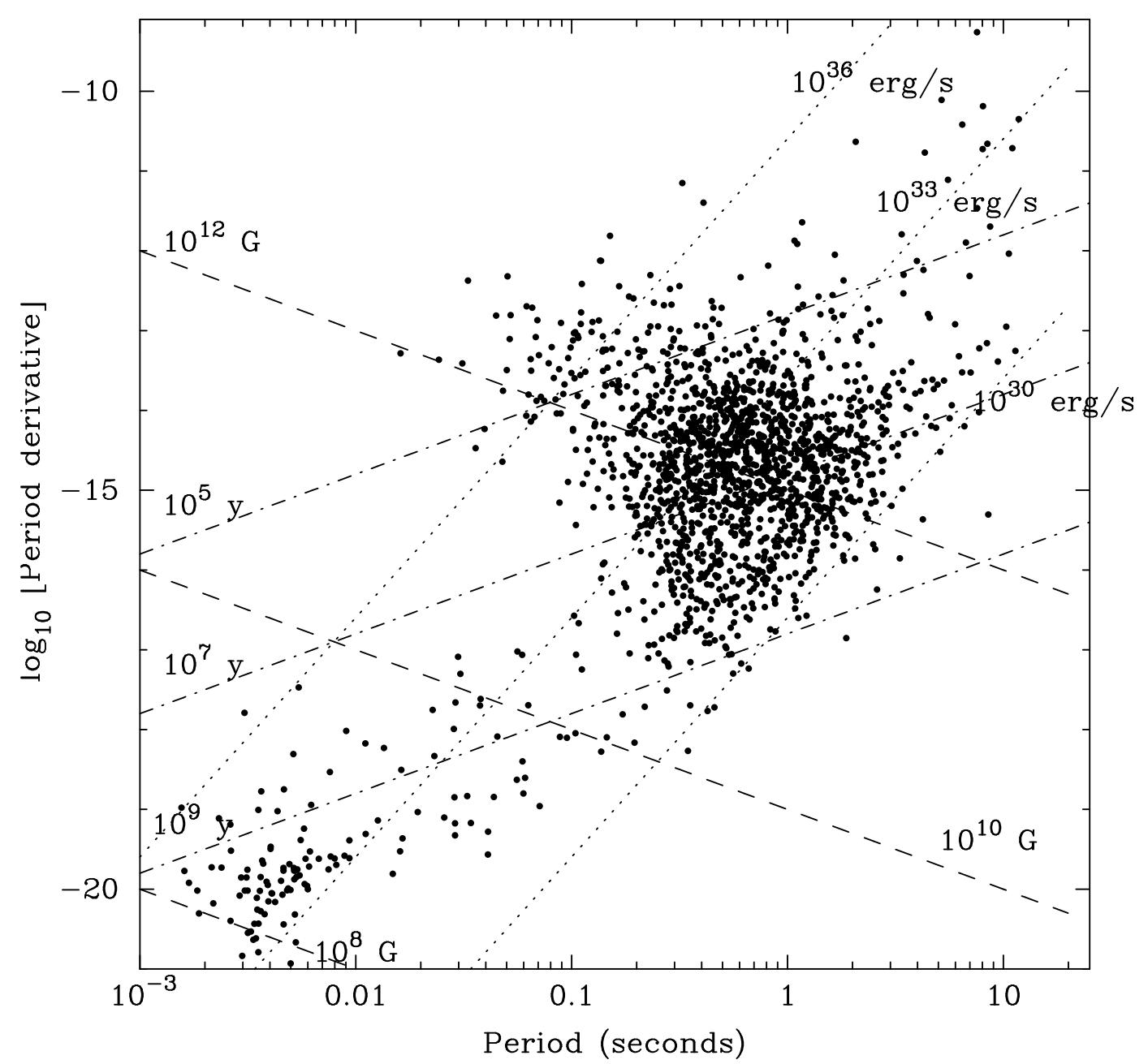

Figure 1.3: The measured distribution of $P$ and $\dot{P}$ for all known pulsars. Lines of constant characteristic age (dot-dash), constant magnetic field (dash), and constant spindown luminosity (dot) are shown. Data are taken from the ATNF pulsar catalog (Manchester et al. 2005).

$10^{6}$ and $10^{8}$ years. The spin-down luminosities of normal pulsars cover a wide range, with young pulsars $\left(\tau_{\mathrm{c}}<10,000 \mathrm{yrs}\right)$ being the most energetic.

All normal pulsars have their astrometric and spin properties measured through pulsar timing and later are used for population studies. In addition, some are individually interesting. For example, PSR J1740-3052 is a young pulsar orbiting around an $11 M_{\odot}$ star (Stairs et al. 2001), PSR B1718-19 is the first non-recycled pulsar found in a globular cluster (Lyne et al. 1993), and PSR B0531+21 shows 
nanosecond duration bursts of radio emission called giant pulses (Hankins et al. 2003). Only a small fraction of normal pulsars are in binary systems; there will be a reverse of this trend when millisecond pulsars are examined.

\subsubsection{Millisecond Pulsars}

Millisecond pulsars (MSPs) are defined here to have periods less than $20 \mathrm{~ms}^{1}$. As seen in Figure 1.3, MSPs have $\dot{P}$ around $10^{-20} \mathrm{~s} \mathrm{~s}^{-1}, B$ between $10^{8}$ and $10^{9}$ G, and $\tau_{\mathrm{c}}$ between $10^{9}$ and $10^{12}$ years. The first MSP was discovered by Backer et al. (PSR B1937+21; 1982) and was the fastest rotating pulsar until the recent discovery of PSR J1748-2446ad (Hessels et al. 2006). These can be seen at the bottom left corner of Figure 1.3.

Most MSPs are found in binary systems. They are thought to form after a pulsar's radio emission ceases and enters the pulsar graveyard (lower right corner of $P-\dot{P}$ diagram). Next the companion overflows its Roche lobe and begins to accrete matter onto the pulsar. The accretion spins the pulsar up (decreases the pulsar's spin period) through the transfer of angular momentum to periods on the order of a few to tens of milliseconds. Some pulsars are only partially spun up and make up the stream of pulsars on the $P-\dot{P}$ diagram that connects the two populations together. They are known as partially recycled pulsars.

The stage between being a dead pulsar and a MSP is when the pulsar is part of a low-mass X-ray binary (LMXB). No radio emission is seen from the pulsar

\footnotetext{
${ }^{1}$ There is no true formal definition to a MSP, some authors define them to be pulsars with periods less than $10 \mathrm{~ms}$, while others impose a limit of $30 \mathrm{~ms}$.
} 
during its time as a LMXB. The recent discovery of PSR J1023+0038 (Archibald et al. 2009) has provided us with observational evidence of this transition from a LMXB to a radio pulsar. The origin of isolated MSPs is unknown but they could result from either the companion's supernova disrupting the binary system or the MSP destroying the companion using the wind from the pulsar as seen in black widow systems (Ruderman et al. 1989). Other MSP binary systems like the triple system in M3 (Backer et al. 1993), the highly eccentric Galactic binary system PSR J1903+0327 (Champion et al. 2008), and the pulsar planetary system PSR B1257+12 (Wolszczan \& Frail 1992) pose more unanswered questions as to how MSP binary systems evolve.

Millisecond pulsars are known to be much more stable rotators than normal pulsars. This stability allows us to measure their properties more precisely than that of normal pulsars. This fact has motivated large campaigns to find as many MSPs as possible with many goals in mind. Among these goals are finding the fastest spinning pulsar to help put constraints on the neutron star EoS, tests of general relativity (Taylor et al. 1992), and the detection of gravitational waves (Hobbs et al. 2010).

\subsubsection{Rotating Radio Transients}

The first rotating radio transients (RRATs) were discovered in a re-processing of the Parkes Multi-beam Pulsar Survey (PMPS) using a single-pulse search and 11 of these were presented in their discovery paper (McLaughlin et al. 2006). Since 
then about 56 RRATs in total have been discovered $^{2}$ and the single-pulse search used to detect them has become a part of any standard search pipeline (Cordes \& McLaughlin 2001).

When RRATs were initially discovered, their exact nature was not known. With more observations, it became apparent that RRATs were neutron stars with emission properties that deviated somehow from those of normal pulsars. Many theories have been put forward to explain the RRATs including radio emission being disrupted by the fall back of supernova material (Li 2006), trapped plasma being released from radiation belts (Luo \& Melrose 2007), and circumstellar material interacting with the pulsar (Shannon \& Cordes 2008). A less radical idea is proposed by looking at observations of PSR B0656+14 (Weltevrede et al. 2006). If this pulsar were located at 12 times its current distance, it too would only be found in a singlepulse search.

\subsection{Pulsar Searching}

Pulsar searching is a very computationally demanding task that requires detecting signals with unknown periodicity and dispersion measure in noisy data sets. A brief description of the standard algorithm is discussed in the following sections.

\subsubsection{De-dispersion}

The ISM causes a frequency dependent time delay (Equation 1.8) on the pulsar's signal across the bandwidth (shown in Figure 1.1). This effect decreases our

\footnotetext{
${ }^{2}$ http://www.as.wvu.edu/ pulsar/rratalog/
} 
sensitivity to the pulsar's signal. This effect can be corrected for by breaking up the bandpass into multiple frequency channels and adding the channels together, correcting with the appropriate time delay. This process is known as de-dispersion.

When searching for pulsars, the DM is unknown at the time of the search and many trial DMs must be tried for a given time series of data. As the DM value increases, scattering and DM smearing lower the effective time resolution, so it is optimal to choose DM steps with this in mind. A sensible choice is to make the dispersive delay time a multiple of the sampling time $\left(\tau_{\text {samp }}\right)$. Using this as a guide and inserting $\tau_{\text {samp }}$ into equation 1.10, the $i^{\text {th }}$ DM is given by

$$
\mathrm{DM}_{i}=1.205 \times 10^{-7} \mathrm{~cm}^{-3} \operatorname{pc}(i-1) \tau_{\text {samp }}\left(f^{3} / \Delta f\right),
$$

where $f$ and $\Delta f$ are in $\mathrm{MHz}$ and $\tau_{\text {samp }}$ is in ms.

When the effective time smearing due to scattering and DM smearing becomes greater than $\tau_{\text {samp }}$, the data can be decimated without loss of sensitivity to short period pulsars. This is done by simply adding consecutive time samples together and allows for faster processing of the resulting time series.

\subsubsection{Frequency Domain Searches}

Though the first pulsars were discovered in time domain searches looking for individual pulse trains, it quickly became apparent that searches in the frequency domain would be fruitful due to the periodic natures of pulsars. Time series data are transformed into the frequency domain via a Fast Fourier Transform (FFT). 
Figure 1.4 shows a time series and its resulting FFT. The pulsar is not visible in the time domain, but shows up easily in the frequency domain.

To improve the sensitivity of searches, low frequency correlated red noise that commonly shows up in the FFT of a time series can be removed. This can be removed by calculating a running mean, subtracting the mean, and setting the standard deviation to unity.

For pulsars with narrower pulse widths, the higher harmonics may be used to help detect the pulsar using harmonic summing (Taylor \& Huguenin 1969). This is done by stretching the FFT by factors of two and adding them together. The noise increases by a factor of $\sqrt{2}$ but the pulsar's signal doubles, resulting in a net gain of $\sqrt{2}$ in $\mathrm{S} / \mathrm{N}$.

RFI can also be mitigated in the Fourier domain. Frequencies of known RFI and their harmonics can be identified and removed from the data by replacing the corresponding FFT bins with the local mean to eliminate their effects on the data. This is known as birdie zapping and can also be used to eliminate the signal from a strong pulsar that may be overwhelming other potential candidates in a given data set. RFI removal is discussed more in section 1.4.5.

\subsubsection{Time Domain Searches}

The first pulsars were discovered through their single pulses but this technique fell out of favor when it was realized that frequency domain searches are more effective for most pulsars. Presented in Nice et al. (1999), the single-pulse search was 
revived, resulting in the discovery of Rotating Radio Transients (RRATs; Mclaughlin et al. 2006), and now is common in most pulsar search pipelines.

The single-pulse search is accomplished by looking for events in a time series above a certain threshold. For purely Gaussian noise, the number of pulses expected by chance above some threshold $S / N_{\min }$ is

$$
n\left(>S / N_{\min }\right) \sim 2 n_{\text {samples }} \int_{S / N_{\min }}^{\infty} \exp \left(-x^{2}\right) \mathrm{d} x
$$

where $n_{\text {samples }}$ is the number of samples in the time series. Requiring $n<3$ usually leads to $S / N_{\min }=4$. In practice usually $S / N_{\min }$ is chosen to be 5 or 6 because RFI may exist in the time series, making the distribution non-Gaussian. Since the signal may be smeared due to multiple time samples occurring during a pulse, the data are smoothed (i. e. consecutive samples added together) many times and each smoothed time series is searched for events above the chosen threshold. Duplicate events that occur at the same time in many smoothed time series are removed and diagnostic plots like Figure 1.5 are produced.

\subsubsection{Acceleration Searches}

Isolated pulsars show no change in their period within the time frame of a single observation, but pulsars in binary systems can show a change in their period during a single observation due to orbital motion caused by a Doppler shift. This change in period results in power being spread across multiple bins in the frequency domain, hence reducing our sensitivity to them. Early techniques to correct for 
possible (and unknown!) changes in period with time involved resampling the time series with a constant acceleration and then FFTing the data (Anderson 1992). This requires a new FFT for each acceleration tried and is computationally expensive. This method has been used for many years resulting in the discovery of many binary systems (e. g. Camilo et al. 2000).

A newer technique (and the one used in this work) is to do the correction entirely in the frequency domain and does not require a new FFT to search each acceleration for a given DM value. An inverse finite impulse response filter is applied to the Fourier coefficients which effectively gathers the power into a single Fourier bin (Ransom et al. 2002). The exact form of this filter depends on the center frequency of the Fourier bin and the number of bins being swept up into one bin. This technique is equivalent to the time domain acceleration correct but computationally is much faster.

\subsubsection{Radio Frequency Interference}

Radio frequency interference (RFI) occurs in most astronomical observations even with the dedicated work of telescope personnel and the government to limit its effect on observations. Many techniques have been developed to combat RFI that can be used in various stages of data processing. One, already mentioned in Section 1.4.2, is birdie zapping, where known RFI frequencies are removed from the frequency domain data.

Another common tactic is to eliminate the RFI in the raw data by applying 
a mask to the data. This mask is created by looking at the raw data and finding events in time or frequency that have intensities above a certain threshold, which can be adjusted by the user. Using this method a whole frequency channel can be ignored if the interference is narrowband or a small chunk of data in the time domain if the RFI is just present for a small period of time. This mask is applied to the raw data and smooths out any places in the data that deviate significantly from the mean.

\subsubsection{Candidate Selection}

After each DM for a given observation has been searched, many thousands of candidates are found that need further culling before being looked at by a human eye. At this point, a list of candidates can be narrowed down by putting restrictions that each candidate has to be in a certain period range, a certain DM range that depends on the observation's position on the sky, or that a candidate must be detected in multiple consecutive DMs. After this winnowing, the candidates are ranked by some significant factor (usually signal-to-noise ratio), and the best candidates are then revisited to create diagnostic plots to be examined by eye (shown in Figure 1.6). Figures 1.7, 1.8, and 1.9 show examples of a weakly detected pulsar, noise, and RFI. The most common plots include a folded pulse profile at the detected period, a plot of profile significance (i.e. $\chi^{2}$ ) versus DM, and a two-dimensional plot of intensity versus frequency and pulse phase.

For each of the above mentioned plots, certain characteristics should be seen. 
For a folded pulse profile, binning the time domain data by the period of the pulsar, the profile will have a signal-to-noise ratio about the same as the Fourier amplitude found in the Fourier domain search and for most pulsars the pulse width will be less then $10 \%$ of the pulse period. In the plot of profile significance versus DM, the peak should occur at a non-zero DM near that of the most significant detected DM in the Fourier domain search. A peak at zero DM means the signal is terrestrial and is not a pulsar. The plot of intensity versus frequency and pulse phase should generally show signal across the full frequency range of the observation, since pulsars are coherent emitters. Exceptions to the rules exist for most pulsars and a legitimate candidate can only be identified by using all the information available in a given diagnostic plot.

\subsection{Pulsar Timing}

Pulsar timing is a powerful technique which uses the periodic nature of a pulsar to measure its properties, the companion's properties (for binaries), and even the properties of the ISM along the line of site to the pulsar. A pulsar must have an integer number of pulses between each observed pulse and pulsar timing uses this fact to produce high precision measurements of pulsar parameters that are unparalleled in other astronomical and many other physical observations.

Pulsar timing uses times of arrival (TOA) to obtain the properties of the pulsar, binary system, and the ISM along the line of site. The TOAs reference some fiducial point in the pulse profile, which is determined by comparing the observed 
pulse profile to some standard template, a noiseless pulse profile that mimics the observed profile. This may be done in the time domain, but analysis has shown that this can be done more precisely in the frequency domain by Fourier transforming both the observed profile and template and sifting the Fourier transforms to match using $\chi^{2}$ minimization (Taylor 1992). This shift is then added to the start time of the observations of the pulse nearest to the center of the observation to measure a TOA.

Each parameter measurable from pulsar timing is related to a time delay that has a different signature. Figure 1.10 shows examples of the functional form when a parameter is unmodeled or off by a small percentage and these functions can be related to time delays as a function of time and/or ecliptic latitude. Pulsar timing uses a $\chi^{2}$ minimization process to match the TOA with the closest integer pulse in the model. A commonly used package for pulsar timing is $\mathrm{TEMPO}^{3}$ and this package is the one used for the work presented in this dissertation. TEMPO requires a list of TOAs known as a "tim" file and a parameter "par" file as its input (see Tables 1.1 and 1.2 for examples). The parameters for the par files are taken from the discovery observations and the value of $\dot{P}$ is initially set to zero.

Pulsar timing is an iterative process and phase-coherent solutions, where all pulses are accounted for, often require a considerable amount of work. A standard procedure for timing an isolated pulsar follows. From a discovery observation, a rough estimate for the spin period, position, and DM can be obtained. For a starting point, three or four observations need to be taken within a week, with at least two

\footnotetext{
${ }^{3}$ http://www.atnf.csiro.au/research/pulsar/tempo/
} 


\begin{tabular}{lcc}
\hline Parameter & Value & Error \\
\hline PSRJ & J1939+2134 & \\
RAJ & $19: 39: 38.558720$ & $2 \times 10^{-6}$ \\
DECJ & $+21: 34: 59.13745$ & $3 \times 10^{-5}$ \\
DM & 71.0398 & $2 \times 10^{-4}$ \\
PEPOCH & 47899.5 & \\
F0 & 641.9282611068082 & $1 \times 10^{-12}$ \\
F1 & $-4.331749 \mathrm{E}-14$ & $9 \times 10^{-20}$ \\
PMRA & -0.46 & $2 \times 10^{-2}$ \\
PMDEC & -0.66 & $2 \times 10^{-2}$ \\
POSEPOCH & 47500 & \\
F2 & $4.0 \mathrm{E}-26$ & $2 \times 10^{-27}$ \\
RM & -10 & 9 \\
PX & 0.12 & $8 \times 10^{-2}$ \\
DM1 & -0.0012 & $1 \times 10^{-4}$ \\
EPHVER & 2 & \\
\hline
\end{tabular}

Table 1.1: Parameter file for PSR J1939+2134 created using the ATNF pulsar catalog. For a listing of each parameter's definition see the TEMPO website (http://tempo.sourceforge.net).

observations on consecutive days if possible. This will form the basis to obtain a very precise measurement of the pulse period and the effects of a position error will be negligible on this short of a time scale. The next observation should occur about a week later, then a further one two weeks later. This will allow a phase coherent solution to be maintained while fitting for a least one additional parameter, usually one of the positions (right ascension or declination).

At this point I would like to remind the reader there is not a single path to obtaining a phase coherent solution and fitting for one parameter or another first will not change the final outcome. After the initial set of close observations, it is common to have monthly observations and add fitting parameters as the solution begins to show any systematics. One measured parameter, DM, can be added into a 


\begin{tabular}{lrcrr}
\hline $\begin{array}{l}\text { One digit } \\
\text { site code }\end{array}$ & $\begin{array}{r}\text { Frequency } \\
(\mathrm{MHz})\end{array}$ & $\begin{array}{c}\text { TOA } \\
(\mathrm{MJD})\end{array}$ & $\begin{array}{r}\text { Error } \\
(\mu \mathrm{s})\end{array}$ & $\begin{array}{r}\mathrm{DM} \\
\left.(\mathrm{pc} \mathrm{cm})^{-3}\right)\end{array}$ \\
\hline 1 & 350.024 & 54635.1855866360913 & 4724.34 & 58.9692 \\
1 & 350.024 & 54635.3270274613273 & 1218.34 & 58.9692 \\
1 & 350.024 & 54635.3305967224120 & 763.82 & 58.9692 \\
1 & 350.024 & 54635.3336626022885 & 1633.34 & 58.9692 \\
1 & 350.024 & 54635.3368200142955 & 1161.67 & 58.9692 \\
1 & 820.024 & 54649.1010862025466 & 2295.77 & 56.4605 \\
1 & 820.024 & 54651.0756557255164 & 4225.02 & 56.4606 \\
1 & 820.024 & 54655.0880919218542 & 2031.62 & 56.4608 \\
1 & 820.024 & 54660.3650753525734 & 1397.15 & 61.1662 \\
1 & 820.024 & 54662.0587921884389 & 1073.28 & 61.1662 \\
1 & 820.024 & 54672.0115769060159 & 2182.13 & 61.1669 \\
1 & 820.024 & 54672.0136819386650 & 1918.02 & 61.1669 \\
1 & 820.024 & 54672.0603119562970 & 1393.80 & 61.1669 \\
1 & 820.024 & 54712.9446744331688 & 2875.19 & 61.1702 \\
1 & 990.024 & 54741.9785042888195 & 1184.31 & 62.3980 \\
1 & 820.024 & 54771.8042972519088 & 1751.32 & 61.1738 \\
1 & 820.024 & 54788.9349653136534 & 823.57 & 61.1741 \\
1 & 820.024 & 54826.8261669399877 & 1229.77 & 61.1731 \\
1 & 820.024 & 54885.5935857104819 & 1604.78 & 61.1685 \\
1 & 820.024 & 54917.4351944806540 & 773.25 & 61.1660 \\
1 & 820.024 & 54947.3577423199548 & 862.03 & 61.1645 \\
1 & 820.024 & 54976.2348945594538 & 1476.65 & 61.1641 \\
\hline & & & & \\
\hline
\end{tabular}

Table 1.2: An example TEMPO TOA files for PSR J2111+2108 showing one year's worth of TOAs. The TOAs are in Princeton format. The one digit site code is a character assigned to each observatory with 1 representing the GBT, frequency is the center frequency of the observation, TOA is the measured time when the pulse is recorded, error is the measured error on the TOA, and DM is the dispersion measure of the pulsar when it was observed. 
timing model at any point in the process as long as multiple frequency observations have been obtained. DM can be fitted most easily at the beginning of the process when the spin period is the only other parameter to be fitted or at the end when all other parameters have been accounted for.

A few techniques can be used that will minimize the uncertainties in a timing model before doing pulsar timing. The first is a process known as gridding and is used to refine the position of a pulsar. In this process, five observations are taken of a given pulsar, with the same integration times and occur on source (discovery position) and in each of the four cardinal directions with the offset being about onehalf the telescope beam width at that frequency. Each time series is de-dispersed and folded at the period of the pulsar. Along with right ascension and declination these profiles are used for determining the most likely position of the pulsar. The weighted means for right ascension and declination were computed using the reduced $\chi^{2}$ for each profile. If a pulsar is not detected in an observation, it is not used in the weighted mean.

For MSPs a better determination of DM can be obtained from a single observation where there is a large time delay compared to the period between the highest and lowest frequencies of the bandpass. This is done by simply breaking the bandpass into four separate frequency channels, creating an individual TOA for each of the four frequencies, and using TEMPO to fit these four TOAs with DM as the only unfixed parameter. This will result in about an order of magnitude more precise DM than what can be acquired using reduced $\chi^{2}$ optimization in the time domain. 
The difference between timing an isolated normal pulsar and an isolated millisecond pulsar is that the number of pulses are much higher for millisecond pulsars and losing a single rotation of the pulsar is much easier to do. This can easily be overcome by properly scheduling the timing observations.

Binary pulsars need more prior information before pulsar timing can be done with them. They require at least three more parameters to be fitted and in general must be fitted from the start of the timing procedure. The additional parameters, known as the Keplerian parameters, are the orbital period $P_{b}$, projected semi-major axis $x$, epoch of periastron passage $T_{o}$, orbital eccentricity $e$, and longitude of periastron, $\omega$. Of the five parameters, $P_{b}, x$, and $T_{o}$ must be known prior to timing a pulsar, and $e$ and $\omega$ are unmeasurable for many binary pulsars because their orbits are circularized during recycling.

One can use Kepler's third law and obtain a mass function for the binary system

$$
f\left(m_{\mathrm{p}}, m_{\mathrm{c}}\right)=\frac{\left(m_{\mathrm{c}} \sin i\right)^{3}}{\left(m_{\mathrm{p}}+m_{\mathrm{c}}\right) 2}=\frac{4 \pi^{2}\left(a_{\mathrm{p}} \sin i\right)^{3}}{G P_{b}^{2}}=\frac{4 \pi^{2} x^{3}}{T_{\odot} P_{b}^{2}},
$$

where $m_{\mathrm{p}}$ and $m_{\mathrm{c}}$ are the masses of the pulsar and its companion, $i$ is the orbital inclination angle, $a_{\mathrm{p}}$ is the size of the pulsar orbit around a common center of mass, $G$ is the gravitational constant, and $T_{\odot}$ is a constant $=\frac{G M_{\odot}}{c^{3}}=4.925490947 \mu \mathrm{s}$. The mass function is usually expressed in solar masses. Assuming a canonical pulsar mass of $1.4 M_{\odot}$, and $\mathrm{i}=90^{\circ}$ a minimum mass of a companion can be determined and this information can be used to plan further observations in radio and other 
wavelengths. A first-order estimate can be made obtained for the binary parameters by fitting a sine wave as a function of time to the solar system barycentered periods of each observation of the pulsar. This will allow for the determination of the spin period of the pulsar, the orbital period of the pulsar, the projected semi-major axis of the binary system, and the epoch of periastron. Figure 1.11 show an example of this fitting procedure for recycled pulsar PSR J2222-0137.

Along with binary parameters, post-Keplerian parameters, or general relativistic effects on the binary system's orbit, can be measured for some binary system. Expressed in their lowest post-Newtonian order, they are as follows:

$$
\begin{gathered}
\dot{\omega}=3 T_{\odot}^{2 / 3}\left(\frac{P_{b}}{2 \pi}\right)^{-5 / 3} \frac{1}{1-e^{2}}\left(m_{\mathrm{p}}+m_{\mathrm{c}}\right)^{2 / 3}, \\
\gamma=T_{\odot}^{2 / 3}\left(\frac{P_{b}}{2 \pi}\right)^{1 / 3} e \frac{m_{\mathrm{c}}\left(m_{\mathrm{p}}+2 m_{\mathrm{p}}\right)}{\left(m_{\mathrm{p}}+m_{\mathrm{c}}\right)^{4 / 3}}, \\
r=T_{\odot} m_{\mathrm{c}}, \\
s=\sin i=T_{\odot}^{-1 / 3}\left(\frac{P_{b}}{2 \pi}\right)^{-2 / 3} x \frac{\left(m_{\mathrm{p}}+m_{\mathrm{c}}\right)^{2 / 3}}{m_{\mathrm{c}}}, \\
\dot{P}_{b}=-\frac{192 \pi}{5} T_{\odot}^{5 / 3}\left(\frac{P_{b}}{2 \pi}\right)^{-5 / 3} f(e) \frac{m_{\mathrm{p}} m_{\mathrm{c}}}{\left(m_{\mathrm{p}}+m_{\mathrm{c}}\right)^{1 / 3}},
\end{gathered}
$$

where all masses are in solar units and 


$$
f(e)=\frac{\left(1+(73 / 24) e^{2}+(37 / 96) e^{4}\right)}{\left(1-e^{2}\right)^{7 / 2}} .
$$

In the above expressions $\dot{\omega}$ is the relativistic advance in periastron, $\gamma$ is the Einstein delay, $r$ and $s$ give the Shapiro range and shape, and $\dot{P}_{b}$ is the orbital decay due to gravitational waves being emitted. Only a small fraction of binary systems have any observable post-Keplerian parameters with the double pulsar, PSR J0737-3039A/B, and PSR B1534+12 being the only systems to have all five measurable (Kramer et al. 2006). $\dot{\omega}$ is the most common parameter to be measurable.

A full description of the mathematics, formulae, and time corrections needed to do pulsar timing is behind the scope of this dissertation. For the interested reader a good introduction can be found in the Handbook of Pulsar Astronomy (Lorimer \& Kramer 2005) and references therein.

\subsection{Outline of Thesis}

The goals of this work are to apply the techniques discussed in this chapter and future chapters to advance our knowledge of the pulsar population in a variety of ways. Chapter 2 will discuss the observations and discovery of thirteen pulsars in the GBT 350-MHz drift-scan survey. Chapter 3 will present some recent timing observations of RRATs and a brief discussion comparing them to the normal pulsar population. Chapter 4 will present an analysis of normal period pulsars in globular clusters and draw a conclusion on their origin. Chapter 5 will summarize the work presented in this dissertation and suggest future work. 

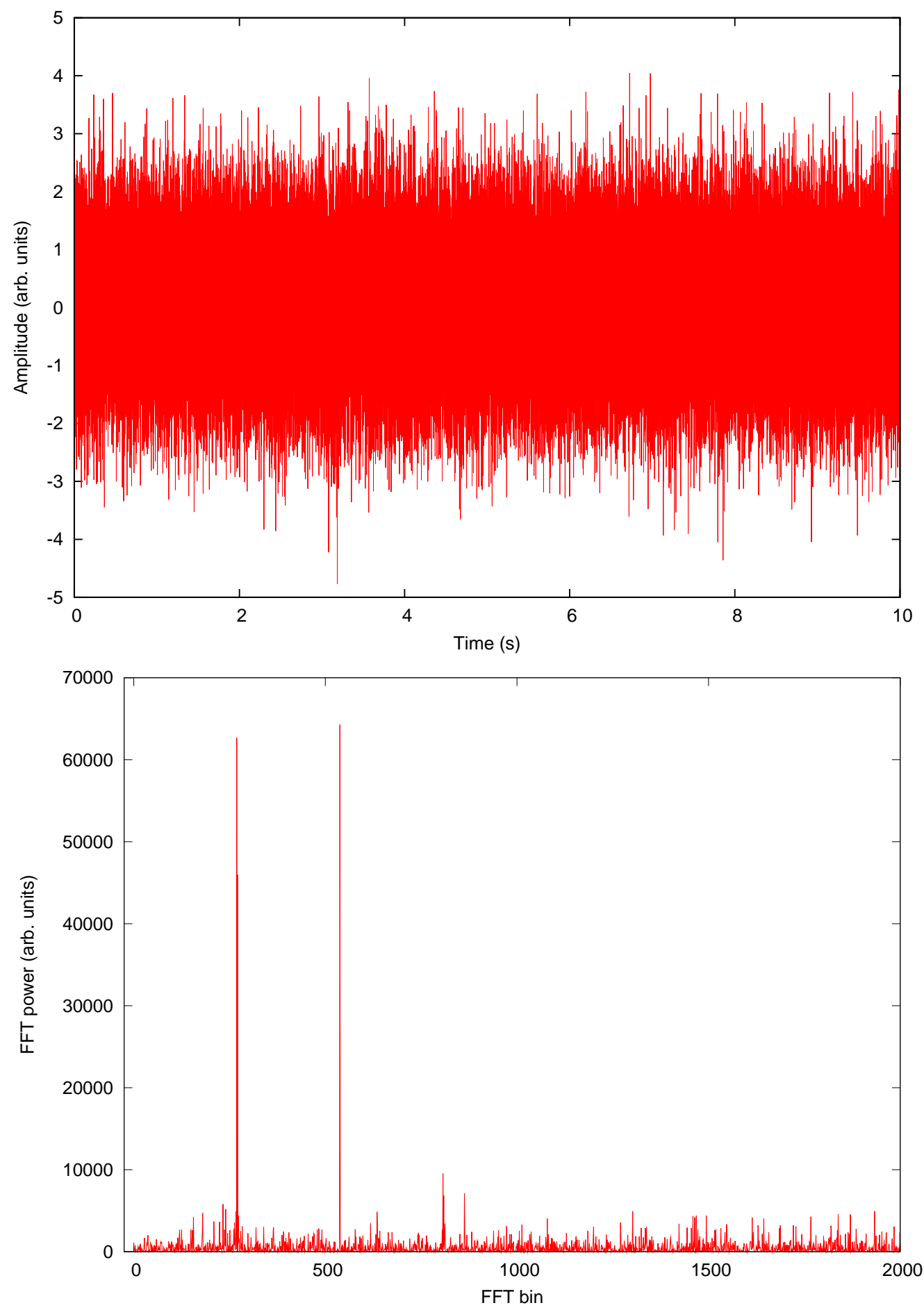

Figure 1.4: Top: Time series of a fake pulsar created with a period of $0.975 \mathrm{~s}$ and a pulse width of $24 \%$. The individual pulses can not be seen. Bottom: FFT of the time series with only the first 2000 FFT bins shown for clarity. The first and second harmonics are clearly visible. 
Single pulse results for 'single_J1754-30_55078_0001'

Source: $1754-30$

Telescope: GBT

Instrument: GUPP|

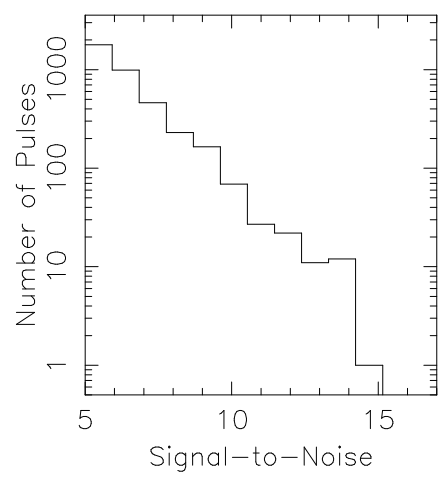

$N$ samples: 7311360

Sampling time: $245.76 \mu \mathrm{s}$

Freq $_{c t r}: 350.0 \mathrm{MHz}$
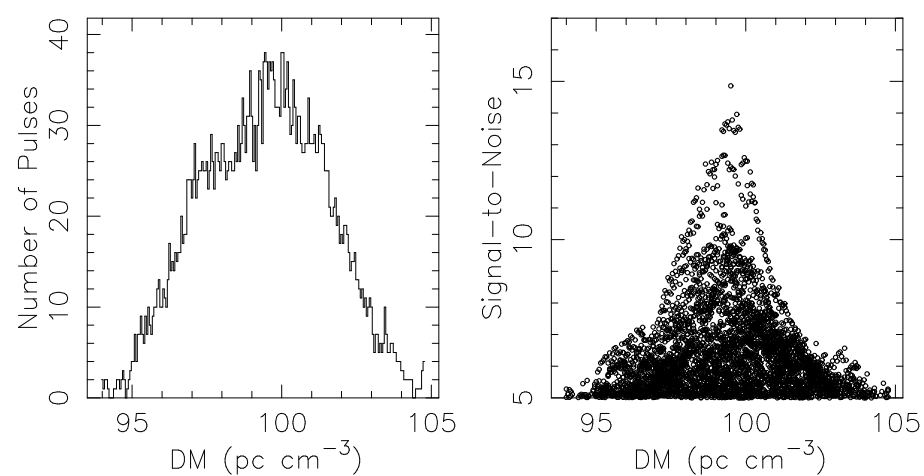

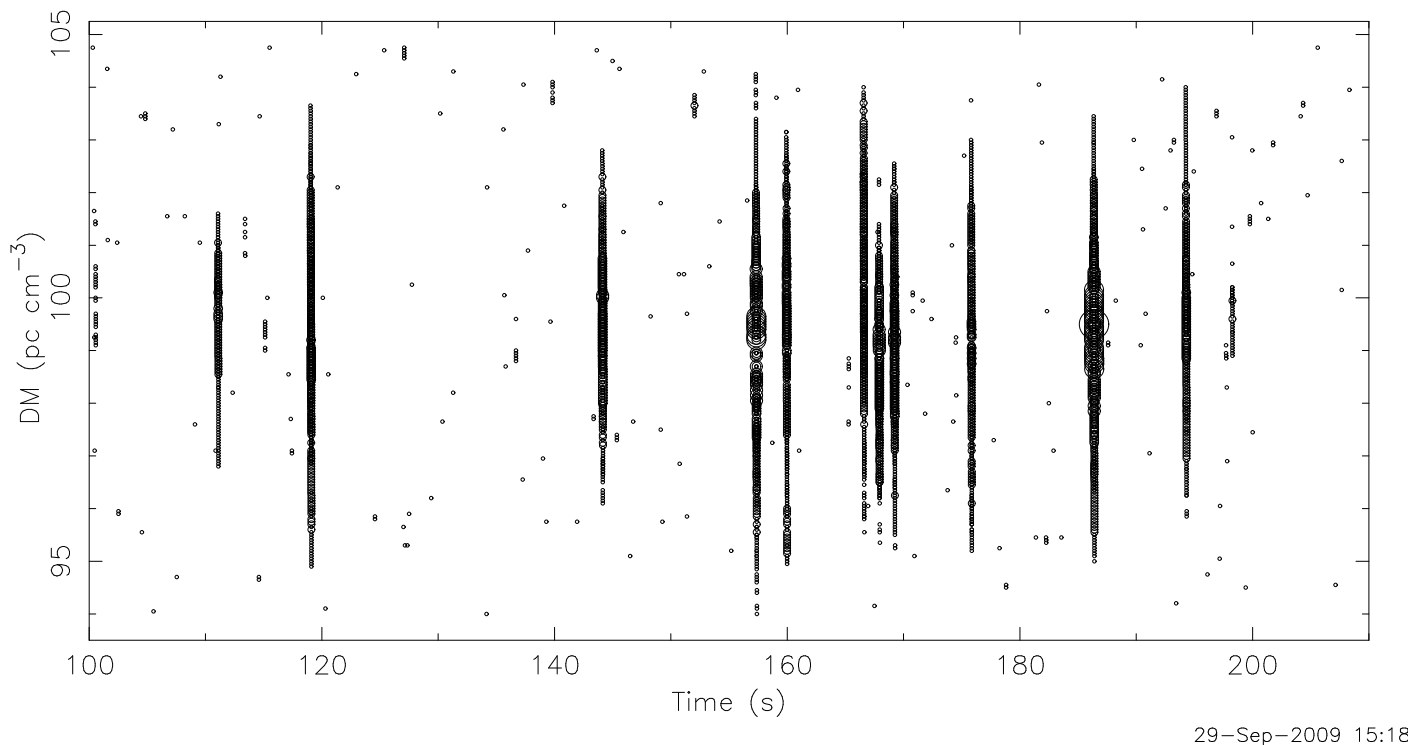

Figure 1.5: Single pulse search output of PSR J1754-30. The top middle and top right plots show excess number of detections at the DM of the pulsar while the bottom plot shows detections as a function of time and DM with circle size being proportional to signal-to-noise of the pulse. In this observation, about a dozen pulses can be seen centered around a DM of $99 \mathrm{pc} \mathrm{cm}^{-3}$.

The top left shows a histogram of the number of detected pulses as function of signal-to-noise. 


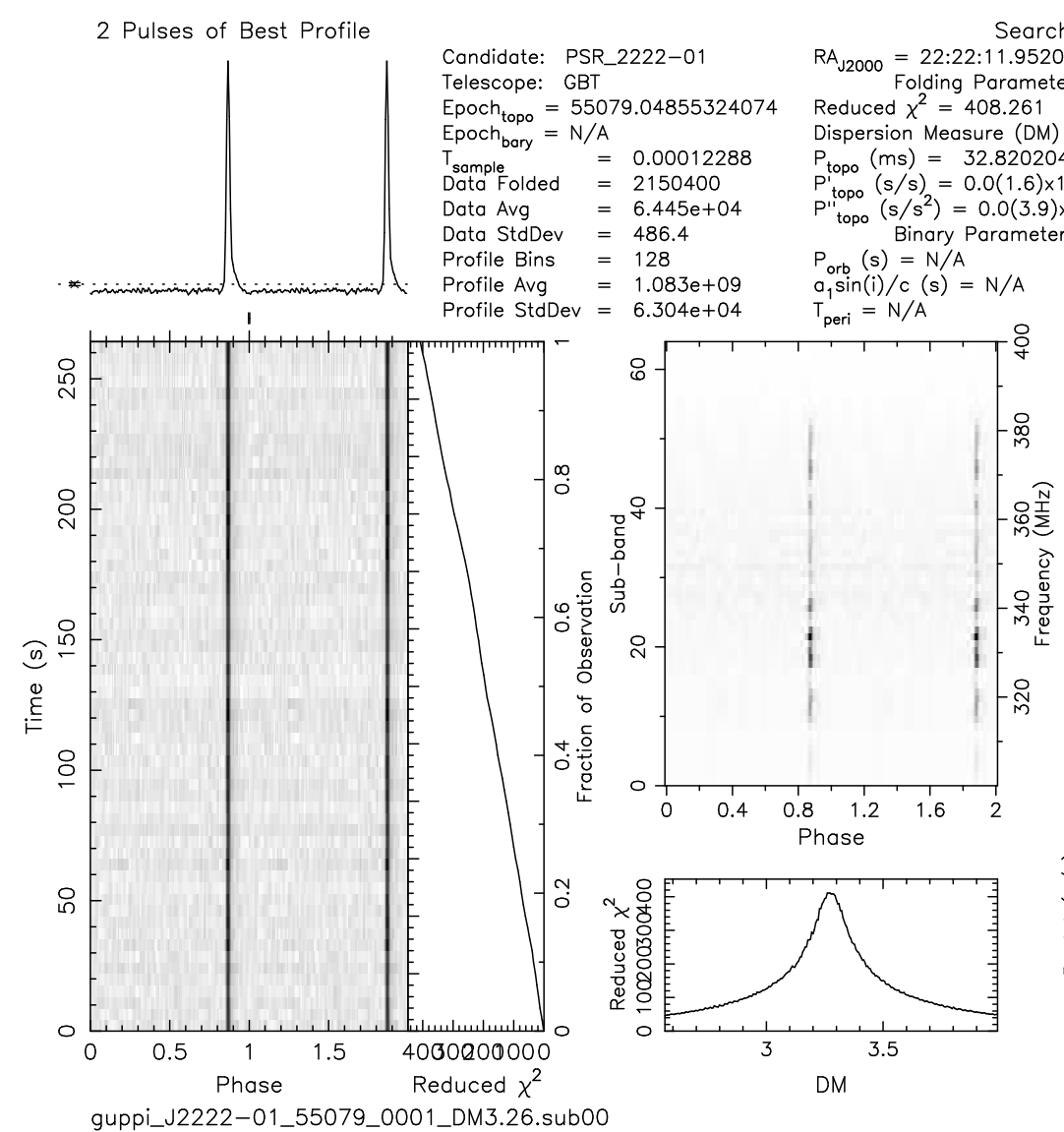

Search Information

$\operatorname{DEC}_{J 2000}=-01: 38: 56.4000$

oise) 0

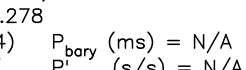

$P^{\prime}$ bary $(s / s)=N / A$
$P^{\prime \prime}\left(s / s^{2}\right)=N / A$

$e=N / A$

$\omega(\mathrm{rad})=\mathrm{N} / \mathrm{A}$
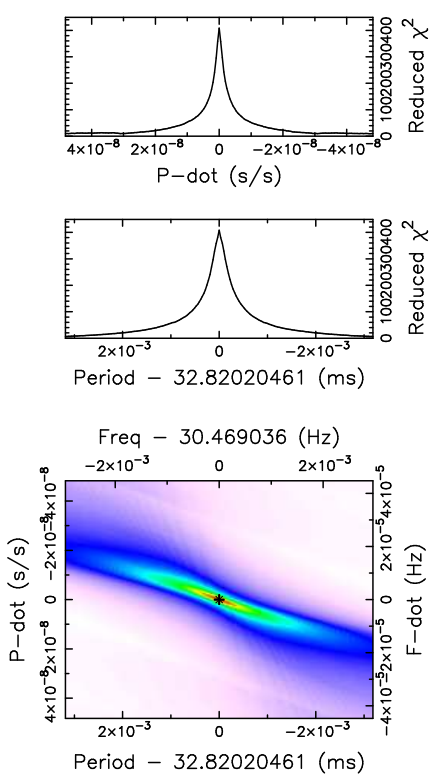

jboyles 10-Sep-2009 16:39

Figure 1.6: A set of diagnostic plots for an observation of PSR J2221-01. The left hand side shows a pulse profile at the top and a plot of intensity versus time and pulse phase on the bottom. Along the plot of intensity versus time is a plot of $\chi^{2}$ versus time. The middle top plot shows frequency versus pulse phase and the bottom shows $\chi^{2}$ versus DM. The right hand side (from top to bottom) shows $\chi^{2}$ versus period, $\chi^{2}$ versus $\dot{P}$, and an intensity plot of period- $\dot{P}$ phase space. 

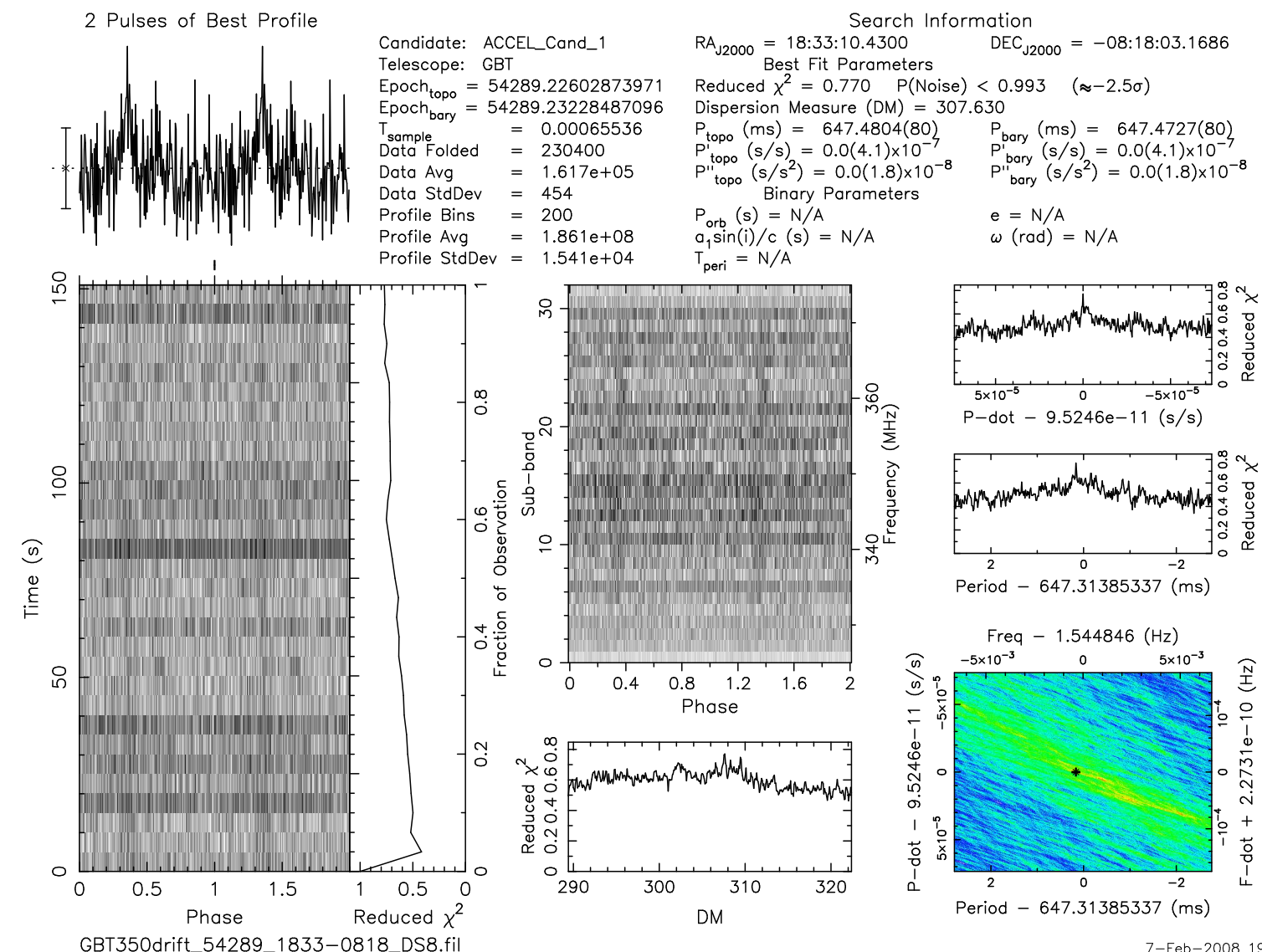

$=1.541 \mathrm{e}+04$

$\mathrm{T}_{\text {peri }}=\mathrm{N} / \mathrm{A}$
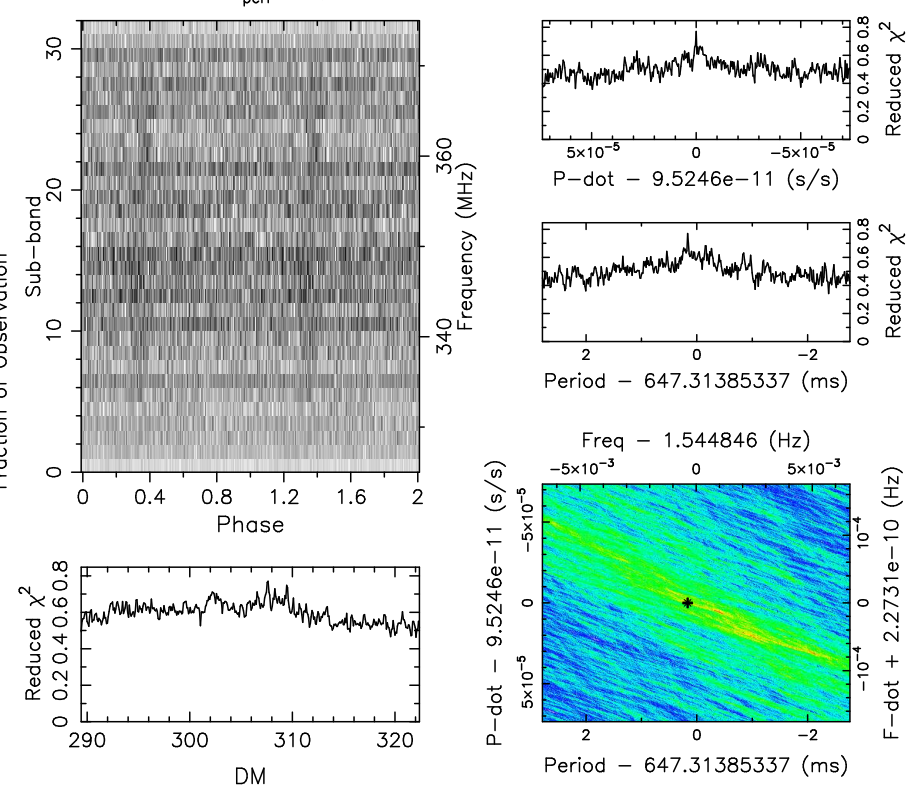

Figure 1.7: An example of a weak redetected pulsar in the $350-\mathrm{MHz}$ drift-scan survey. PSR B1829-08 has a period of $647 \mathrm{~ms}$, a DM of $301 \mathrm{pc} \mathrm{cm}^{-3}$, and a 400$\mathrm{MHz}$ flux of $9.9 \mathrm{mJy}$. Pulse scattering has reduced the pulsar's signal to almost an undetectable level. 


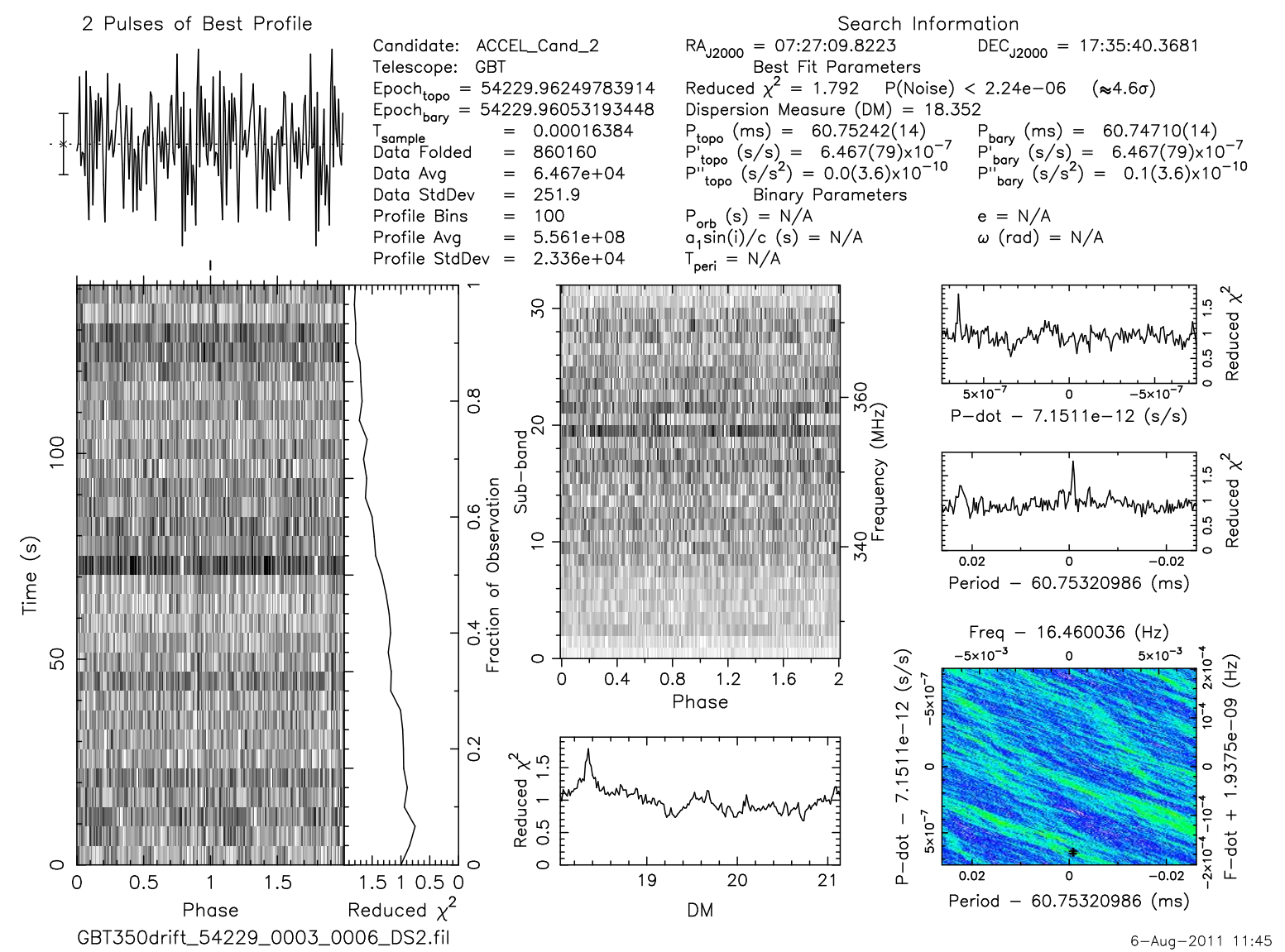

Figure 1.8: Example of pure noise showing none of the characteristics of a detected pulsar. 


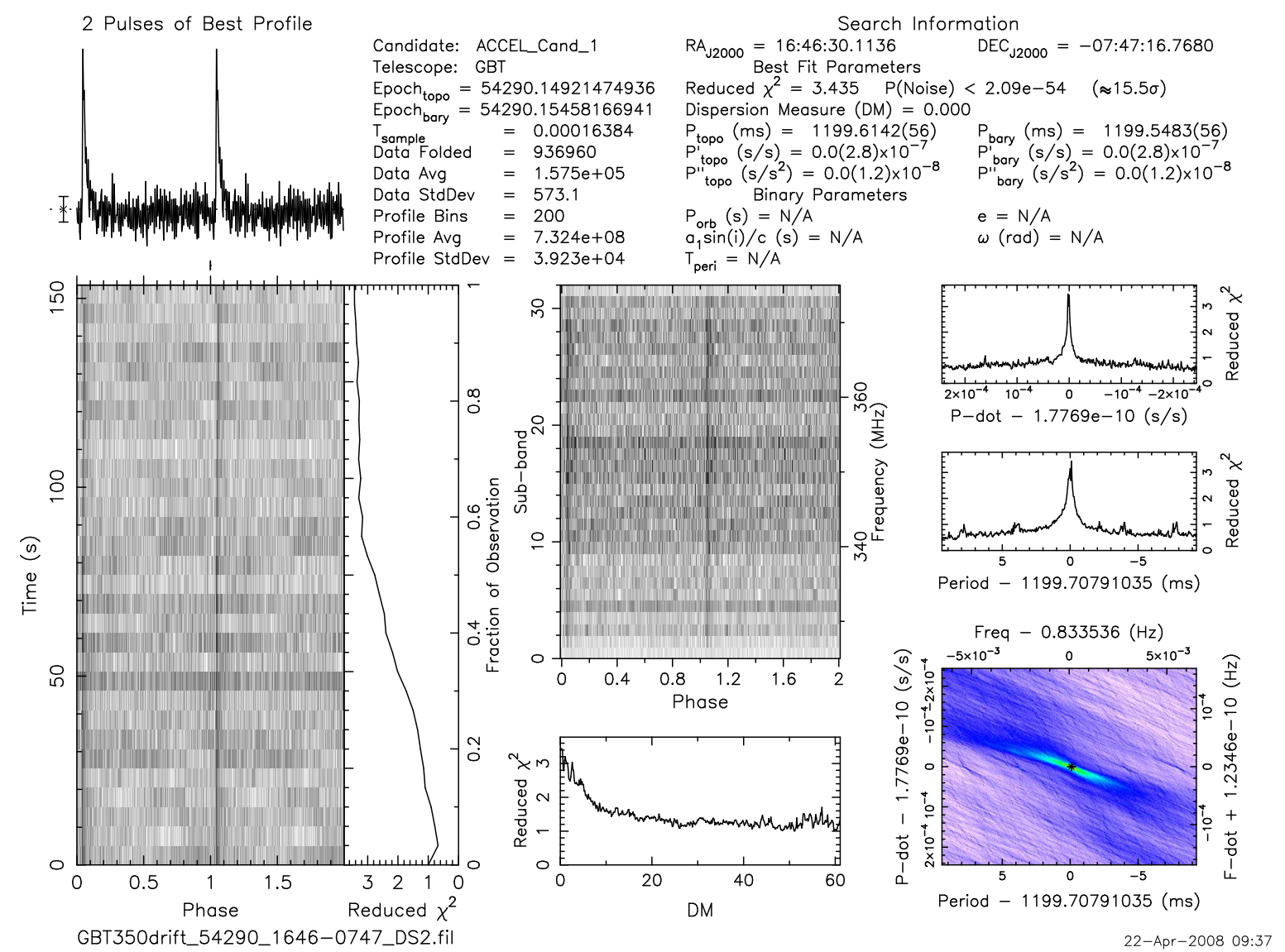

Figure 1.9: A diagnostic plot showing a $1.2 \mathrm{~s}$ RFI signal. Besides the plot of significant versus DM, this plot shows all the characteristics of a pulsar. 

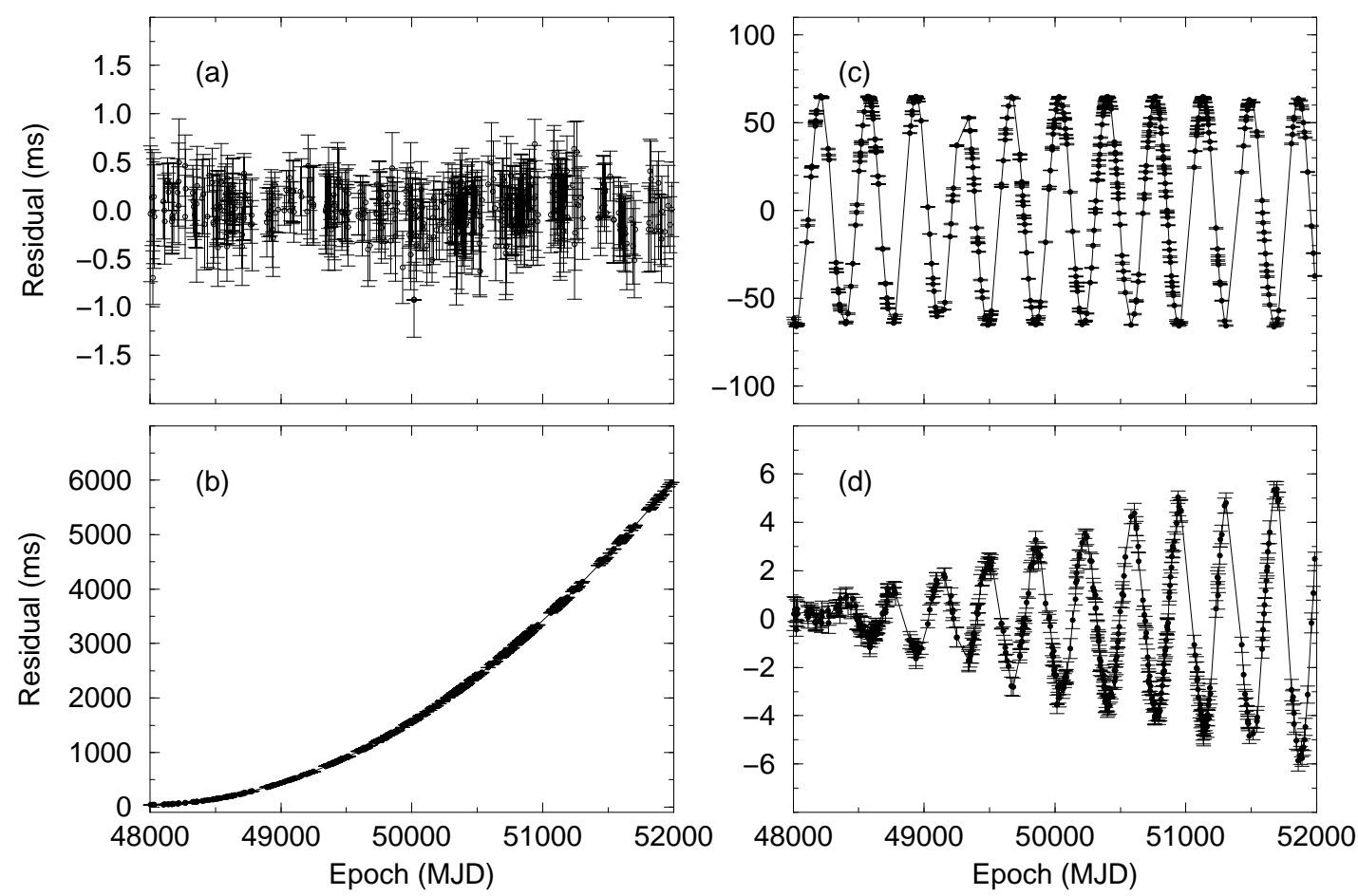

Figure 1.10: Timing residuals for PSR B1133+16. (a) Shows a timing model with no systematics and a random distribution of TOAs, indicating a good model fit. (b) Timing model showing the parabolic signature of underestimating $\dot{P}$ by $4 \%$. (c) Timing model showing the constant amplitude yearly sinusoidal signature of a offset in position (here 1 arcmin in declination). (d) Timing model showing the linearly increasing amplitude, yearly sinusoidal signature of not including proper motion in the timing model. Figure used with permission from the Handbook of Pulsar Astronomy (Lorimer \& Kramer 2005). 


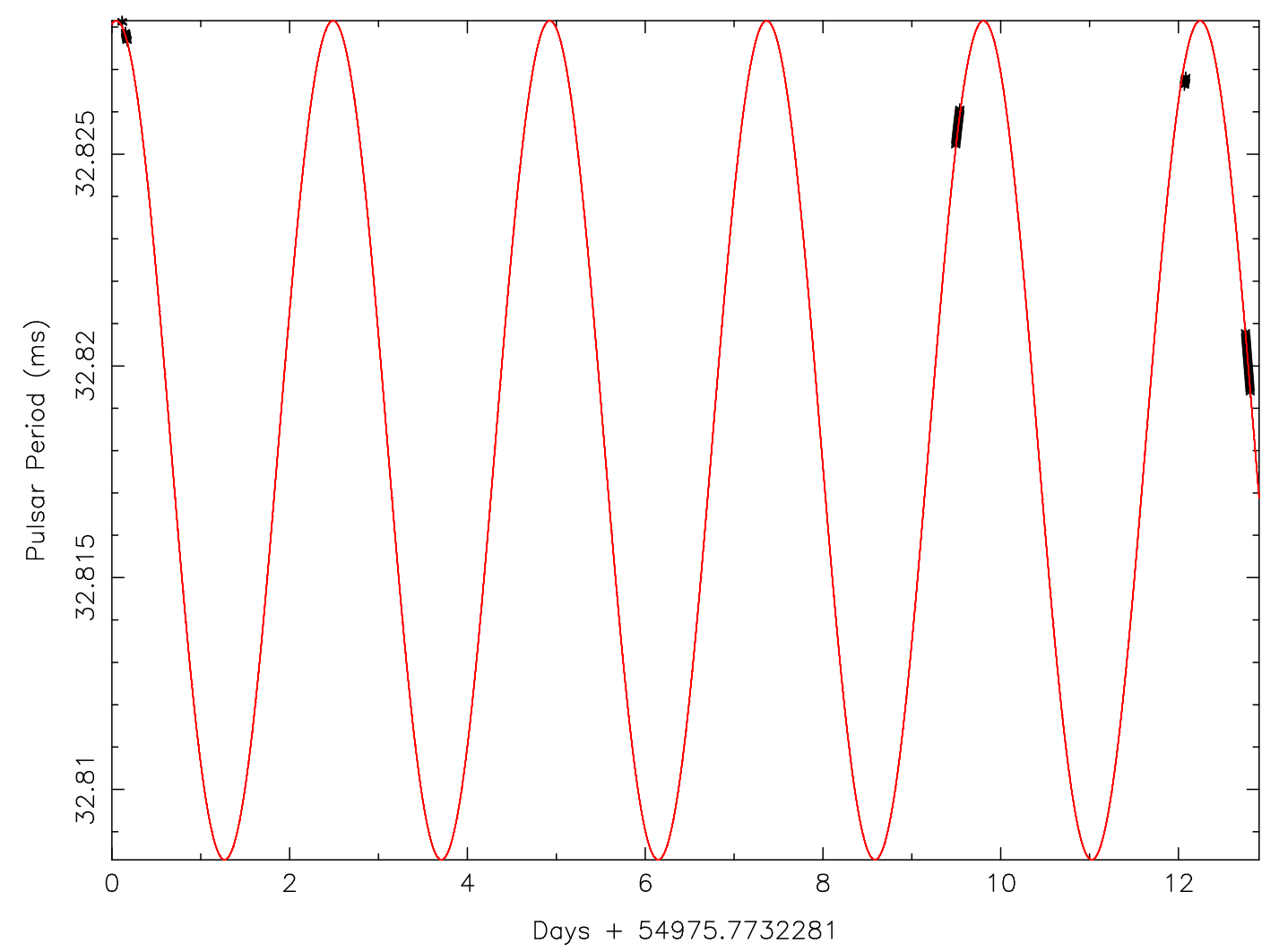

Figure 1.11: A sinusoidal fit to the barycentered data for PSR J2222-0137 with an orbital period of 2.45 days and a semi-major axis of 10.8 lt-s. The fit is slightly imperfect due to the pulsar's non-zero orbital eccentricity. 


\section{Chapter 2}

\section{A 350-MHz Drift-scan Pulsar Survey with the GBT.}

\subsection{Introduction}

Since the discovery of the first pulsar in 1967 (Hewish et al. 1968), many fascinating discoveries and experiments have been associated with pulsars. These include but are not limited to tests of general relativity (Lyne et al. 2004), studies of binary evolution (Phinney 1993), probes of electron density models of the interstellar medium (Cordes \& Lazio, 2002), constraints on the neutron star equation of state (Lattimer \& Prakash 2001), and use in a pulsar timing array (PTA) for gravitational wave detection (Hobbs et al. 2010).

Searches for pulsars are very demanding, requiring both significant amounts of computational and human resources. The high time and frequency resolution needed for pulsar searches result in data rates greater than for most astronomical projects (typically $100 \mathrm{~GB} / \mathrm{hr}$ or more). Large computing clusters are needed to thoroughly search for both periodic and transient dispersed signals. After all the computer searching is complete, candidates are typically inspected with the human eye to determine legitimate pulsar candidates, though automated algorithms, such as neural nets, are becoming increasingly important for sifting through large numbers of candidate signals (e.g., Eatough et al. 2010).

Most modern large scale non-targeted pulsar surveys (e. g. Parkes Multibeam 
Pulsar Survey; Manchester et al, 2001, High Time Resolution Universe Pulsar Survey; Bailes et al. 2010, PALFA; Cordes et al. 2006) are conducted around $1.4 \mathrm{GHz}$ and target the Galactic plane. The choice of $1.4 \mathrm{GHz}$ is to limit the effects of the interstellar medium on pulsar observations. Lower frequency observations $(<500$ $\mathrm{MHz}$ ) are better used to locate weak sources away from the Galactic plane where the effects of scattering and dispersion broadening are minimized.

The National Radio Astronomy Observatory (NRAO) at Green Bank, WV has a long history of successful searches for radio pulsars at low frequencies. Damashek et al. (1978) discovered 17 new pulsars with the 92 meter transit telescope at a frequency of $400 \mathrm{MHz}$. Later, using the same telescope at a frequency of $390 \mathrm{MHz}$, Dewey et al. (1985) found 34 new long period pulsars. However, they were not sensitive to newly discovered millisecond pulsars (MSPs; P $<20$ ms; Backer et al. 1982). The first low-frequency survey to be sensitive to MSPs was conducted by Stokes et al. (1985) at $390 \mathrm{MHz}$. While this survey did not discover any MSPs, it did find 20 new long period pulsars. Sayer et al. (1997) used the 43-meter telescope at Green Bank at a frequency of $370 \mathrm{MHz}$ and discovered eight new pulsars including one MSP. New backend hardware and higher data rates now allow frequency resolution that was previously unattainable by previous observing systems. Most recently, a Galactic plane survey was conducted with the Robert C. Byrd Green bank Telescope ${ }^{1}$ (GBT) by Hessels et al. (2008) at $350 \mathrm{MHz}$ and discovered 33 new pulsars, none of which are MSPs. Our survey took advantage of a unique opportu-

\footnotetext{
${ }^{1}$ The Robert C. Byrd Green Bank Telescope (GBT) is operated by the National Radio Astronomy Observatory which is a facility of the U.S. National Science Foundation operated under cooperative agreement by Associated Universities, Inc.
} 
nity when GBT was immobilized for track refurbishing during the northern summer of 2007. During this time, we recorded data at a radio frequency of $350 \mathrm{MHz}$ as the sky passed through the beam of the telescope. We will discuss these drift-scan observations in Section 2. In Section 3 we describe the follow-up observations and timing analysis. In Section 4 we describe the properties of the thirteen pulsars and in Section 5 we describe interesting individual pulsars. Finally in Section 6 we present the conclusions and the future survey planned in light of the results obtained in this survey. The survey sensitivity, pipeline, and the remaining new pulsars will be discussed in Lynch et al. (2012).

\subsection{Survey Observations}

The drift-scan observations occurred from May through August in 2007. The telescope was parked at a set azimuth during the track refurbishing and data were recorded while the sky drifted through the telescope's beam. The elevation of the telescope had a limit of $>60^{\circ}$ during the weekdays and $>30^{\circ}$ during the weekends. The azimuth was set at $\sim 229^{\circ}$ for the first half of the observations and $\sim 192^{\circ}$ for the second half of the observations. These azimuth restrictions allowed us to access declination ranges of $-7.72^{\circ}<\delta<38.43^{\circ}$ and $-20.66^{\circ}<\delta<38.43^{\circ}$ for azimuths of $229^{\circ}$ and $192^{\circ}$, respectively. All right ascensions were available for us to observe.

We chose a frequency of $350 \mathrm{MHz}$ for this survey for many reasons. Firstly, at $350 \mathrm{MHz}$, the radio frequency interference (RFI) environment at the GBT is remarkably manageable. Pulsars generally have very steep spectral indexes (Sieber 


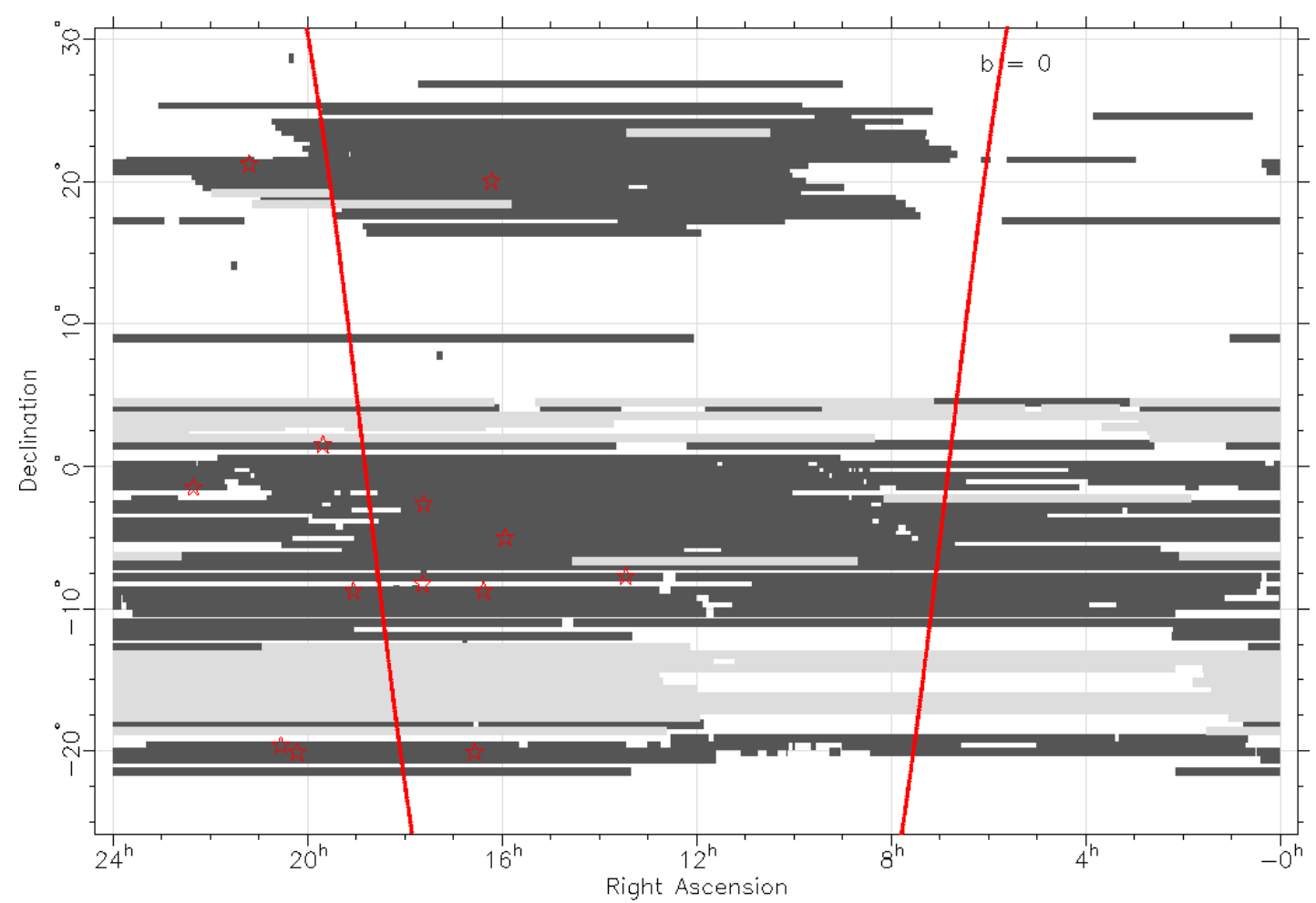

Figure 2.1: A map in equatorial coordinates showing the sky coverage of the survey. The drift-scan portion is shown in black, the PSC portion is shown in gray, and the red line shows the Galactic plane. New discoveries presented in this work are shown as red stars.

1973) making $350 \mathrm{MHz}$ an optimal choice for our survey, which mostly covers high Galactic latitudes, where propagation effects in the interstellar medium are generally less severe. Lastly, the GBT's beam size at $350 \mathrm{MHz}$ is $\sim 36^{\prime}$, allowing us to cover a greater amount of the sky than would be possible with higher frequency receivers. The sky coverage rate (210 $\mathrm{deg}^{2}$ per day) is faster than that achieved by the PMPS at $1.4 \mathrm{GHz}$ (26.25 $\mathrm{deg}^{2}$ per day; Manchester et al. 2001).

The now retired Spigot autocorrelation spectrometer (Kaplan et al. 2005) was used for the GBT drift-scan observations. During the data recording we used $50 \mathrm{MHz}$ of bandwidth with $81.92 \mu$ s sampling time. For the first week of observations (MJDs 54222 - 54230) data were recorded with 1024 three-level auto-correlation functions 
(ACFs) which were accumulated into 16-bit registers for analysis. Afterwards, and until the conclusion of the observations, a new 2048 three-level ACF mode was used which was accumulated into 8-bit registers.

Data were recorded every night on each weekday and for 24 hours a day during the weekends. During this time, 1491 hours of data were taken, amounting to 134 TB (see Table 2.1 for observation list and Figure 2.1). This amounts to a sky coverage of 10347 square degrees, of which $\sim 2800$ went to the Pulsar Search Collaboratory (PSC; Rosen et al. 2010). ${ }^{2}$ A source passing through the center of the beam is visible for roughly $140 \mathrm{~s}$. Assuming a detection significance of $8 \sigma$ and a total system temperature of $80 \mathrm{~K}$ results in a survey flux density limit

$$
\mathrm{S}_{\min } \cong 4.5 \mathrm{mJy} \sqrt{\frac{\epsilon}{1-\epsilon}}
$$

where $\epsilon=W / P$ is the observed pulse duty cycle for a pulse of width $W$ and period $P$. For $\epsilon=0.04$, typical for normal pulsars, this corresponds to a sensitivity limit of roughly 0.9 mJy. Scaling our flux density limit with an average pulsar spectral index of -1.6 (Lorimer et al. 1995) gives us a limit of $0.10 \mathrm{mJy}$ at $1400 \mathrm{MHz}$, about a factor of two less than the PMPS $(\sim 0.16 \mathrm{mJy})$, but our observations are hampered more by dispersion smearing and scattering. A more detailed discussion about the survey sensitivity is presented by Lynch et al. (2012). Data processing was done with the PRESTO³ pulsar package (Ransom 2001).

\footnotetext{
${ }^{2}$ The PSC is a collaboration between NRAO and WVU that involves high-school students in searching for pulsars. http://www.pulsarsearchcollaboratory.com/

${ }^{3}$ http://www.cv.nrao.edu/ sransom/presto/
} 
Table 2.1: Observations

\begin{tabular}{lrccr}
\hline Start & Scan Length & $\begin{array}{c}\text { Azimuth } \\
(\mathrm{d})\end{array}$ & $\begin{array}{c}\text { Elevation } \\
(\text { deg })\end{array}$ & Bits \\
\hline 54222.171759 & 24640 & 229.240 & 60.0 & 16 \\
54223.181169 & 23840 & 229.380 & 60.5 & 16 \\
54224.093518 & 12320 & 229.450 & 61.0 & 16 \\
54224.554143 & 4720 & 229.450 & 61.0 & 16 \\
54224.621643 & 24320 & 229.450 & 61.0 & 16 \\
54224.907592 & 33280 & 229.480 & 35.0 & 16 \\
54225.301921 & 20320 & 229.480 & 35.0 & 16 \\
54225.959525 & 43200 & 229.480 & 35.0 & 16 \\
54226.500116 & 28800 & 229.480 & 35.5 & 16 \\
54226.837373 & 37040 & 229.480 & 35.5 & 16 \\
54227.267766 & 16240 & 229.480 & 35.5 & 16 \\
54227.934375 & 45040 & 229.470 & 71.0 & 16 \\
54228.963969 & 42320 & 229.470 & 61.9 & 16 \\
54229.958403 & 43200 & 229.470 & 61.4 & 16 \\
54230.998090 & 31360 & 229.470 & 73.5 & 8 \\
54231.516273 & 9360 & 229.470 & 66.5 & 8 \\
54231.760058 & 9440 & 229.470 & 66.5 & 8 \\
54231.883657 & 640 & 229.470 & 66.5 & 8 \\
54231.917535 & 25120 & 229.470 & 66.5 & 8 \\
54232.210949 & 24800 & 229.470 & 66.5 & 8 \\
54232.508356 & 44800 & 229.480 & 36.0 & 8 \\
54233.037835 & 39760 & 229.480 & 36.0 & 8 \\
54233.519282 & 44000 & 229.480 & 34.5 & 8 \\
54234.035555 & 36400 & 229.480 & 34.5 & 8 \\
54234.902870 & 44720 & 229.470 & 67.0 & 8 \\
54235.423958 & 2960 & 229.470 & 67.0 & 8 \\
54235.922326 & 45600 & 229.470 & 68.0 & 8 \\
54236.901933 & 47840 & 229.470 & 67.5 & 8 \\
54237.919329 & 46480 & 229.470 & 68.5 & 8 \\
54238.483819 & 44000 & 229.470 & 34.0 & 8 \\
54239.000810 & 40960 & 229.470 & 34.0 & 8 \\
54239.478183 & 43920 & 229.470 & 33.5 & 8 \\
54240.001851 & 42960 & 229.470 & 33.5 & 8 \\
54240.516273 & 39920 & 229.470 & 33.0 & 8 \\
\hline & & & &
\end{tabular}


Table 2.1: cont.

\begin{tabular}{lrccr}
\hline Start & Scan Length & $\begin{array}{c}\text { Azimuth } \\
(\mathrm{deg})\end{array}$ & $\begin{array}{c}\text { Elevation } \\
(\mathrm{deg})\end{array}$ & $\begin{array}{c}\text { Bits } \\
\text { MJD }\end{array}$ \\
\hline 54240.982708 & 41120 & 229.470 & 33.0 & 8 \\
54241.905486 & 27840 & 229.468 & 69.0 & 8 \\
54242.229594 & 19760 & 229.468 & 69.0 & 8 \\
54242.954803 & 43440 & 229.468 & 69.5 & 8 \\
54243.918924 & 46560 & 229.468 & 70.0 & 8 \\
54244.935972 & 49680 & 229.481 & 32.5 & 8 \\
54245.613970 & 11760 & 229.467 & 70.5 & 8 \\
54245.759641 & 32400 & 229.481 & 32.0 & 8 \\
54246.167789 & 25200 & 229.481 & 32.0 & 8 \\
54246.470150 & 47200 & 229.480 & 31.5 & 8 \\
54247.020729 & 4720 & 229.480 & 31.5 & 8 \\
54247.077268 & 32960 & 229.480 & 31.5 & 8 \\
54247.466794 & 42800 & 229.480 & 30.5 & 8 \\
54247.967894 & 42640 & 229.481 & 30.5 & 8 \\
54248.468171 & 46480 & 229.481 & 31.0 & 8 \\
54249.015174 & 38320 & 229.481 & 31.0 & 8 \\
54249.944896 & 2720 & 229.468 & 70.5 & 8 \\
54250.911273 & 47280 & 229.472 & 62.5 & 8 \\
54251.916400 & 46800 & 229.471 & 63.0 & 8 \\
54252.590255 & 31840 & 231.674 & 30.0 & 8 \\
54252.964919 & 42640 & 231.674 & 30.0 & 8 \\
54253.463414 & 44000 & 231.674 & 29.5 & 8 \\
54253.975706 & 41760 & 231.674 & 29.5 & 8 \\
54254.464803 & 44000 & 231.674 & 29.0 & 8 \\
54254.980926 & 41200 & 231.674 & 29.0 & 8 \\
54265.957755 & 43200 & 229.463 & 63.5 & 8 \\
54266.537500 & 3360 & 229.443 & 36.5 & 8 \\
54266.671968 & 24720 & 229.443 & 36.5 & 8 \\
54266.960000 & 42960 & 229.443 & 36.5 & 8 \\
54267.490463 & 11520 & 229.443 & 37.5 & 8 \\
54267.658148 & 960 & 229.443 & 37.5 & 8 \\
54267.759062 & 13520 & 229.443 & 37.5 & 8 \\
54267.929884 & 45600 & 229.443 & 37.5 & 8 \\
54268.467071 & 43200 & 229.471 & 38.5 & 8 \\
\hline & & & &
\end{tabular}


Table 2.1: cont.

\begin{tabular}{lrccr}
\hline Start & Scan Length & $\begin{array}{c}\text { Azimuth } \\
(\mathrm{deg})\end{array}$ & $\begin{array}{c}\text { Elevation } \\
(\mathrm{deg})\end{array}$ & $\begin{array}{c}\text { Bits } \\
\text { MJD }\end{array}$ \\
\hline 54268.968426 & 42320 & 229.471 & 38.5 & 8 \\
54269.909664 & 14480 & 229.463 & 64.0 & 8 \\
54270.095347 & 31440 & 229.463 & 64.0 & 8 \\
54270.937789 & 45040 & 229.463 & 64.5 & 8 \\
54271.945729 & 44320 & 229.463 & 65.0 & 8 \\
54272.991632 & 30560 & 229.472 & 37.0 & 8 \\
54273.370602 & 720 & 229.471 & 37.0 & 8 \\
54273.384676 & 4560 & 229.471 & 37.0 & 8 \\
54273.476377 & 22320 & 229.470 & 38.0 & 8 \\
54273.839005 & 5460 & 229.471 & 38.0 & 8 \\
54273.926203 & 46080 & 229.471 & 38.0 & 8 \\
54274.462858 & 24560 & 229.470 & 39.0 & 8 \\
54274.916898 & 480 & 229.470 & 39.0 & 8 \\
54274.925775 & 240 & 229.470 & 39.0 & 8 \\
54274.929537 & 160 & 229.470 & 39.0 & 8 \\
54274.934016 & 1920 & 229.470 & 39.0 & 8 \\
54274.959525 & 42000 & 229.470 & 39.0 & 8 \\
54275.450104 & 160 & 229.470 & 39.5 & 8 \\
54275.454421 & 3120 & 229.470 & 39.5 & 8 \\
54275.491967 & 6640 & 229.470 & 39.5 & 8 \\
54275.575879 & 33040 & 229.470 & 39.5 & 8 \\
54275.965486 & 6320 & 229.471 & 39.5 & 8 \\
54276.045961 & 35680 & 229.470 & 39.5 & 8 \\
54276.933912 & 45040 & 229.462 & 65.5 & 8 \\
54277.914537 & 46800 & 229.462 & 66.0 & 8 \\
54278.906203 & 47520 & 229.459 & 71.5 & 8 \\
54279.926539 & 45920 & 229.471 & 40.0 & 8 \\
54280.566597 & 34000 & 192.468 & 40.5 & 8 \\
54280.966203 & 42320 & 192.468 & 40.5 & 8 \\
54281.466296 & 12720 & 192.468 & 41.0 & 8 \\
54281.637153 & 26240 & 192.468 & 41.0 & 8 \\
54281.965694 & 42480 & 192.468 & 41.0 & 8 \\
54282.464317 & 640 & 192.467 & 41.5 & 8 \\
54282.518310 & 38000 & 192.467 & 41.5 & 8 \\
\hline & & & &
\end{tabular}


Table 2.1: cont.

\begin{tabular}{lrccr}
\hline Start & Scan Length & $\begin{array}{c}\text { Azimuth } \\
(\mathrm{deg})\end{array}$ & $\begin{array}{c}\text { Elevation } \\
(\mathrm{deg})\end{array}$ & $\begin{array}{c}\text { Bits } \\
\text { MJD }\end{array}$ \\
\hline 54282.968159 & 42320 & 192.468 & 41.5 & 8 \\
54283.916424 & 47040 & 192.457 & 72.0 & 8 \\
54284.899815 & 48320 & 192.468 & 44.0 & 8 \\
54285.464826 & 36000 & 192.467 & 44.5 & 8 \\
54285.885255 & 8000 & 192.467 & 44.5 & 8 \\
54285.987454 & 40800 & 192.468 & 44.5 & 8 \\
54286.915197 & 46800 & 192.461 & 72.5 & 8 \\
54287.477118 & 43200 & 192.450 & 42.0 & 8 \\
54287.986250 & 41280 & 192.450 & 42.0 & 8 \\
54288.471088 & 37520 & 192.450 & 42.5 & 8 \\
54289.467257 & 43200 & 192.467 & 43.0 & 8 \\
54289.977025 & 41600 & 192.468 & 43.0 & 8 \\
54291.115521 & 320 & 192.466 & 51.5 & 16 \\
54291.146539 & 320 & 192.469 & 38.3 & 16 \\
54291.157419 & 400 & 192.463 & 58.8 & 16 \\
54291.183704 & 1200 & 192.469 & 37.5 & 16 \\
54292.121817 & 240 & 192.469 & 37.5 & 16 \\
54292.139722 & 640 & 192.467 & 47.6 & 16 \\
54292.164282 & 320 & 192.470 & 34.0 & 16 \\
54292.175278 & 400 & 192.468 & 43.4 & 16 \\
54292.183299 & 400 & 192.468 & 42.1 & 16 \\
54292.199479 & 400 & 192.468 & 42.1 & 16 \\
54293.268194 & 320 & 192.448 & 80.0 & 16 \\
54293.306574 & 320 & 192.467 & 46.5 & 16 \\
54293.312894 & 400 & 192.470 & 34.4 & 16 \\
54293.324572 & 400 & 192.461 & 65.2 & 16 \\
54293.927188 & 45920 & 192.471 & 30.0 & 8 \\
54294.465220 & 28400 & 192.470 & 30.5 & 8 \\
54294.817917 & 800 & 192.470 & 30.5 & 8 \\
54294.863518 & 80 & 192.470 & 30.5 & 8 \\
54294.872187 & 720 & 192.471 & 30.5 & 8 \\
54294.888507 & 1200 & 192.471 & 30.5 & 8 \\
54294.924201 & 46240 & 192.471 & 30.5 & 8 \\
54295.470521 & 15360 & 192.470 & 31.0 & 8 \\
\hline & & & &
\end{tabular}


Table 2.1: cont.

\begin{tabular}{lrccr}
\hline Start & $\begin{array}{r}\text { Scan Length } \\
\text { MJD }\end{array}$ & $\begin{array}{c}\text { Azimuth } \\
(\mathrm{deg})\end{array}$ & $\begin{array}{c}\text { Elevation } \\
(\mathrm{deg})\end{array}$ & $\begin{array}{c}\text { Bits } \\
\end{array}$ \\
\hline 54295.712188 & 8800 & 192.470 & 31.0 & 8 \\
54295.836157 & 320 & 192.471 & 31.0 & 8 \\
54295.854398 & 160 & 192.471 & 31.0 & 8 \\
54295.859109 & 8720 & 192.471 & 31.0 & 8 \\
54295.969039 & 960 & 192.471 & 31.0 & 8 \\
54296.000903 & 7200 & 192.471 & 31.0 & 8 \\
54296.092211 & 27440 & 192.471 & 31.0 & 8 \\
54296.465394 & 9440 & 192.470 & 31.5 & 8 \\
54296.578426 & 960 & 192.471 & 31.5 & 8 \\
54296.589977 & 28560 & 192.471 & 31.5 & 8 \\
54296.924653 & 2800 & 192.471 & 31.5 & 8 \\
54297.937616 & 45040 & 192.463 & 60.0 & 8 \\
54298.918518 & 30960 & 192.463 & 60.0 & 8 \\
54299.278715 & 15600 & 192.463 & 60.0 & 8 \\
54299.920439 & 16560 & 192.471 & 32.5 & 8 \\
54300.119456 & 29280 & 192.471 & 32.5 & 8 \\
54300.924063 & 46000 & 192.469 & 33.0 & 8 \\
54301.465451 & 42320 & 192.469 & 33.5 & 8 \\
54301.958924 & 43040 & 192.469 & 33.5 & 8 \\
54302.461389 & 43040 & 192.468 & 34.0 & 8 \\
54302.962083 & 43040 & 192.468 & 34.0 & 8 \\
54303.463102 & 43040 & 192.468 & 34.5 & 8 \\
54303.966111 & 43120 & 192.468 & 34.5 & 8 \\
54304.942164 & 44640 & 192.469 & 32.0 & 8 \\
54305.912500 & 47200 & 192.469 & 35.0 & 8 \\
54306.938345 & 45040 & 192.469 & 35.5 & 8 \\
54307.938993 & 44880 & 192.469 & 36.0 & 8 \\
54308.494872 & 40000 & 192.469 & 36.5 & 8 \\
54308.960949 & 43200 & 192.469 & 36.5 & 8 \\
54309.465902 & 33760 & 192.469 & 37.0 & 8 \\
54309.886470 & 6320 & 192.469 & 37.0 & 8 \\
54309.962072 & 42960 & 192.469 & 37.0 & 8 \\
54310.462013 & 43040 & 192.468 & 37.5 & 8 \\
54310.971840 & 42000 & 192.468 & 37.5 & 8 \\
\hline & & & &
\end{tabular}


Table 2.1: cont.

\begin{tabular}{lrccr}
\hline Start & $\begin{array}{r}\text { Scan Length } \\
\text { MJD }\end{array}$ & $\begin{array}{c}\text { Azimuth } \\
(\mathrm{deg})\end{array}$ & $\begin{array}{r}\text { Elevation } \\
(\mathrm{deg})\end{array}$ & Bits \\
\hline 54311.900220 & 44640 & 192.468 & 38.0 & 8 \\
54312.927303 & 45920 & 184.493 & 30.0 & 8 \\
54313.921898 & 46320 & 184.491 & 39.5 & 8 \\
54315.159768 & 25600 & 184.491 & 40.0 & 8 \\
54315.458194 & 44000 & 184.491 & 40.5 & 8 \\
54315.976192 & 41760 & 184.490 & 40.5 & 8 \\
54316.466701 & 34560 & 184.486 & 53.0 & 8 \\
54316.925914 & 41040 & 184.486 & 53.0 & 8 \\
54317.705150 & 22000 & 184.487 & 53.5 & 8 \\
54317.970023 & 42560 & 184.487 & 53.5 & 8 \\
54319.288796 & 12800 & 184.487 & 54.0 & 8 \\
54321.007917 & 10240 & 170.950 & 54.0 & 8 \\
54321.179548 & 24160 & 170.950 & 54.0 & 8 \\
54321.917928 & 46480 & 184.472 & 54.5 & 8 \\
54322.459421 & 46800 & 184.472 & 55.0 & 8 \\
54323.004595 & 39200 & 184.472 & 55.0 & 8 \\
54323.479722 & 5360 & 184.472 & 55.5 & 8 \\
54323.560520 & 23360 & 184.471 & 55.5 & 8 \\
54323.902118 & 5920 & 184.472 & 55.5 & 8 \\
54324.008414 & 38880 & 184.472 & 55.5 & 8 \\
54324.464317 & 39120 & 184.471 & 56.0 & 8 \\
54324.920891 & 4400 & 184.471 & 56.0 & 8 \\
54325.011007 & 38560 & 184.471 & 56.0 & 8 \\
\hline & & & & \\
\hline & & &
\end{tabular}

Table 2.1: Start MJD, scan length, azimuth, elevation, and number of data bits for each observation. 


\begin{tabular}{cccc}
\hline $\begin{array}{c}\text { Low DM } \\
\mathrm{pc} \mathrm{cm}^{3}\end{array}$ & $\begin{array}{c}\text { DM step size } \\
\mathrm{pc} \mathrm{cm}^{3}\end{array}$ & Number of DMs & $\begin{array}{c}\text { Downsample } \\
\text { factor }\end{array}$ \\
\hline 0.0 & 0.03 & 3700 & 2 \\
111.0 & 0.05 & 1700 & 4 \\
196.0 & 0.10 & 1900 & 8 \\
386.0 & 0.20 & 1900 & 16 \\
766.0 & 0.50 & 500 & 32 \\
\hline
\end{tabular}

Table 2.2: Parameters for DMs searched. Each de-dispersed time series was processed using 128 subbands.

\subsection{Discovery Analysis}

These long scans of data then were searched for pulsars. Each scan was broken up into 150 second overlapping beams. Each beam overlapped the next beam by about 70 seconds. The data were downsampled, consecutive samples added together, by factors of $2,4,8,16$, and 32 into multiple files. This was done to increase the search speed at higher DM values. The data were dedispersed at $9700 \mathrm{DM}$ values when processed based on the dispersion smearing that would occur in individual frequency channels. Table 2.2 shows the DM values chosen for processing.

The data were searched for incidences of RFI using the rfifind program from PRESTO. This program compiles a list of the most significant and numerous birdies and also creates a mask which is applied to the raw data before dedispersion. The median and mode of the percentage of data masked due to RFI is $0.56 \%$ and $0.36 \%$ respectively.

The data are dedispersed for the given range of DMs with the RFI mask being applied to the data. This is accomplished by creating 128 frequency subbands and creating time series files in accordance of the DM plan in Table 2.2 with 100 
de-dispersed time series being created at one time.

A single-pulse search was done on the de-dispersed time series files and records all events with a signal-to-noise ratio greater than five sigma. This single-pulse search attempts to find pulses by matched-filtering the data with a series of different width boxcar functions. The matched filtering is accomplished by convolving the boxcars with the full resolution data. The results are stored until the completion of all DMs.

The time series files are then FFTed into the frequency domain. Immediately after the FFT, red noise (low-frequency, correlated noise) was removed from the data. Also in the frequency domain, frequencies that are a common source of RFI are removed from the data. The most common frequency of $60 \mathrm{~Hz}$, due to the power lines, and its harmonics are masked along with a $1.2 \mathrm{~Hz}$ signal and its harmonics that were found later in the data processing.

The frequency-domain power spectrum are searched for harmonic signals. Each series is harmonically stretched and summed with the most significant results stored after each summation. After the completion of all harmonics sums, the results are filtered to exclude duplicate candidates which may occur over multiple harmonics.

Two searches of the Fourier domain are conducted. The first is a non-acceleration search that hereafter will be referred to as a low-acceleration search. This is designed to target long-period pulsars with narrow pulse profiles. The low-acceleration search sums 2, 4, 8, and 16 harmonics for each DM and searches after each summation. All candidates are stored until the completion of all DM values. 
The second search is an acceleration search. This will be referred to as highacceleration search and is designed to look for short-period binary pulsars, binary pulsars with wide pulse profiles, or pulsars in tight binary orbits. The high acceleration search sums 2,4 , and 8 harmonics and searches after each summation. All candidates are stored separately from the low-acceleration candidates until the completion of all DM values.

After the completion of all the searches for each DM, diagnostic plots are created to view the results of the searches. The single-pulse plots are the first to be created. Four DM ranges $\left(0-30\right.$ pc cm ${ }^{-3}, 20-110$ pc cm ${ }^{-3}, 100-310$ pc cm ${ }^{-3}, 300-$ $1015 \mathrm{pc} \mathrm{cm}^{-3}$ ) were chosen to cover all the data with a minimum single-pulse $\mathrm{S} / \mathrm{N}$ $>$ 5.5. An example of an output single-pulse diagnostic plot is shown in Figure 2.2. Sifting is conducted separately for both the low-acceleration search and the high-acceleration search. In the sifting procedure, candidates are removed from the list if the DM is less then one or if the candidate was not seen at least in two consecutive DM values. Next, duplicate entries are removed from the candidate lists. The final step is to sort the remaining candidates by their PRESTO sigma value.

The best 20 low-acceleration candidates and 10 high-acceleration candidates are chosen to produce diagnostic plots. The parameters for each candidate are used as input with the PRESTO program prepfold to create diagnostic plots with the raw data. An example diagnostic plot is shown in Figure 2.3.

After the creation of the diagnostic plots, the associated files for all candidates are compressed and archived to a long-term storage area for human inspection. 
Single pulse results for 'GBT350drift_54287_2111+2114'

Source: unknown

Telescope: GBT

Instrument: SPIGOT
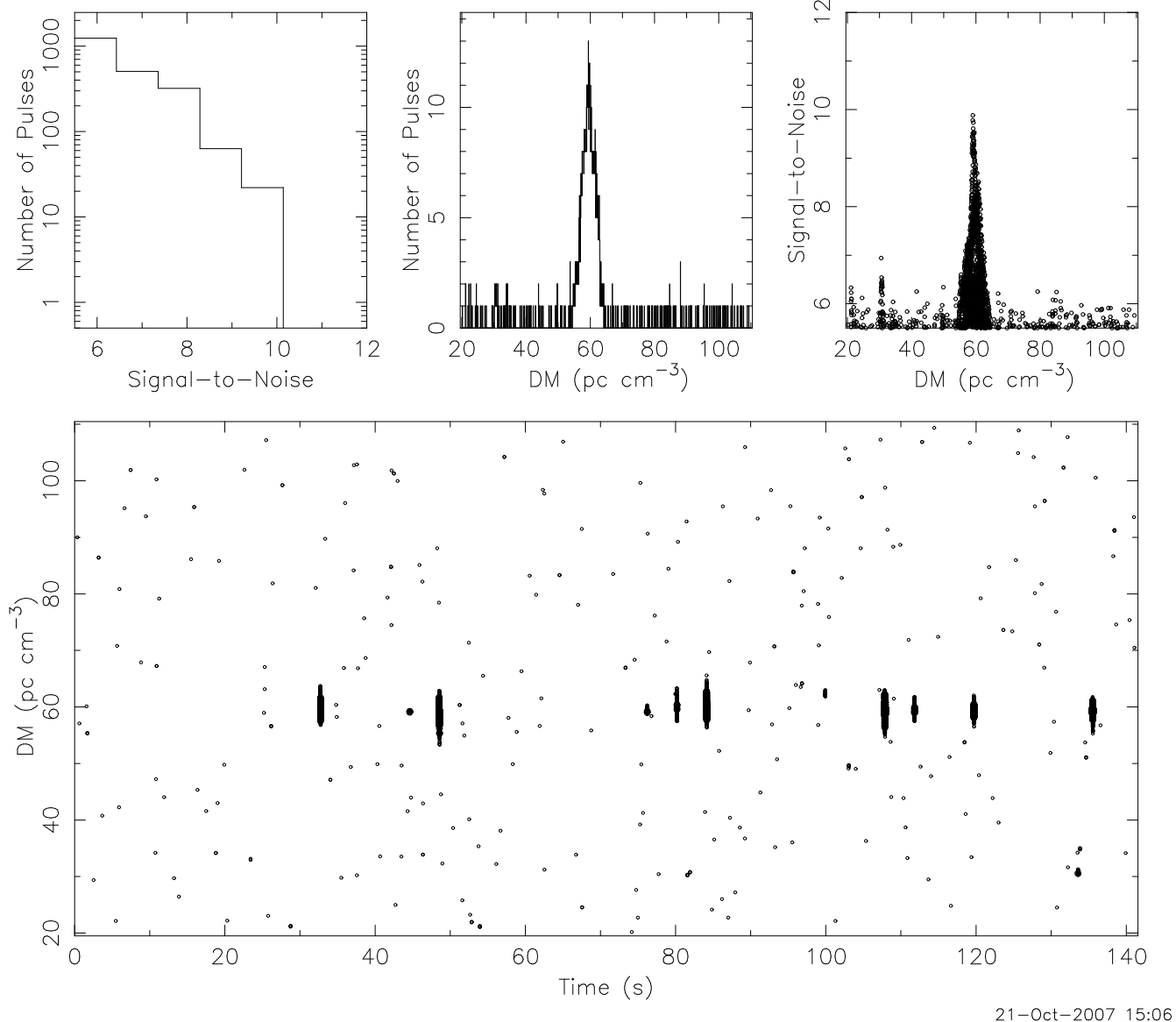

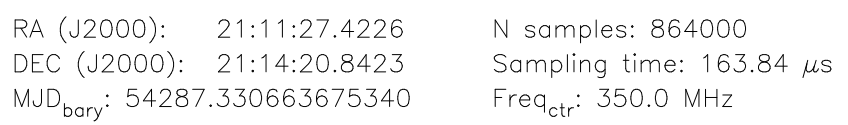

$M J D_{\text {bary }}: 54287.330663675340$

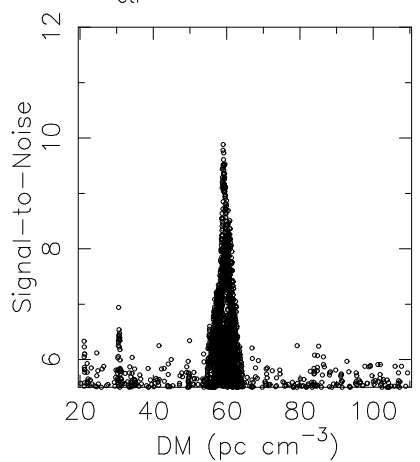

Figure 2.2: Single-pulse search output showing the discovery observation of newly discovered pulsar PSR J2111+2108. 

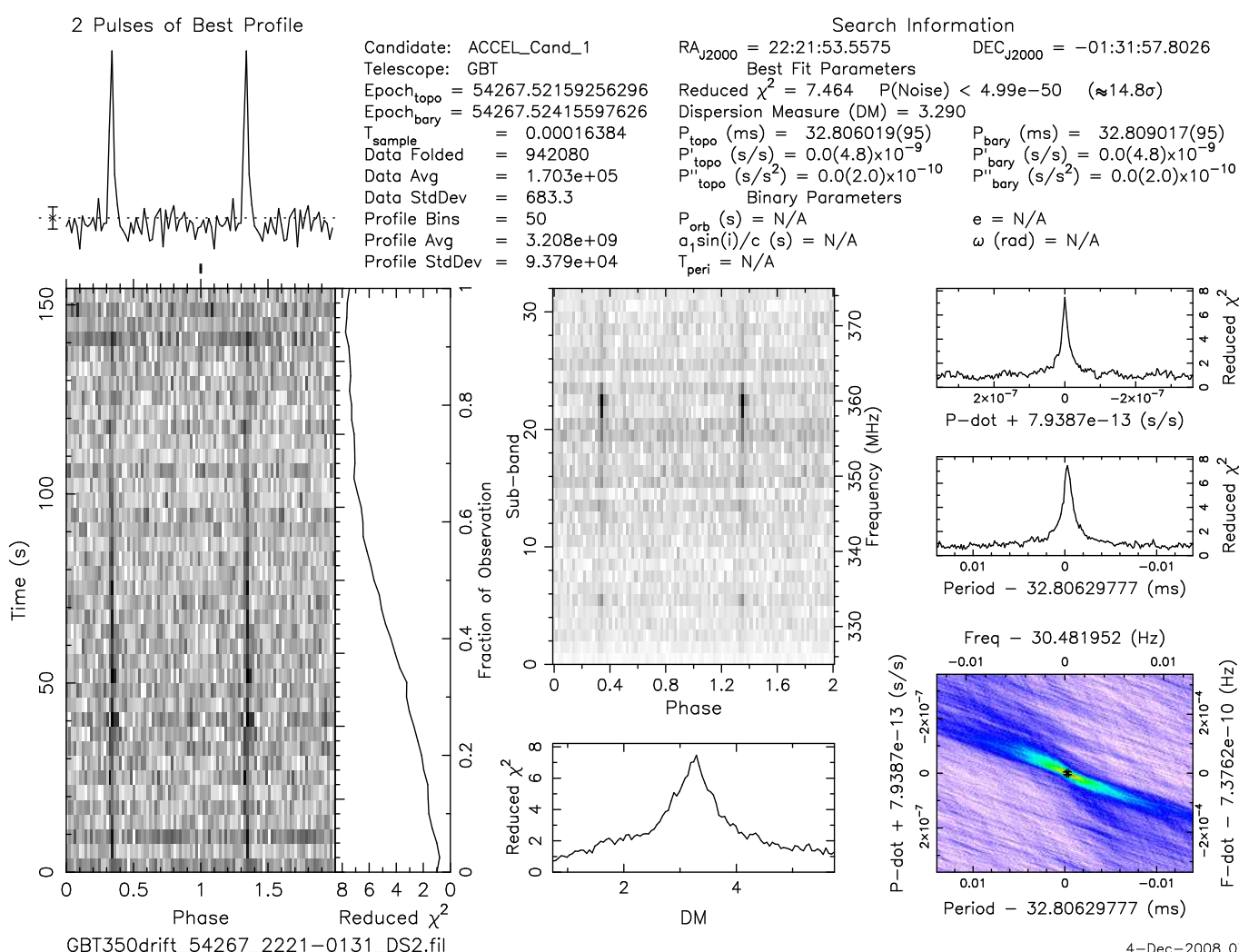

$\mathrm{T}_{\text {peri }}=\mathrm{N} / \mathrm{A}$
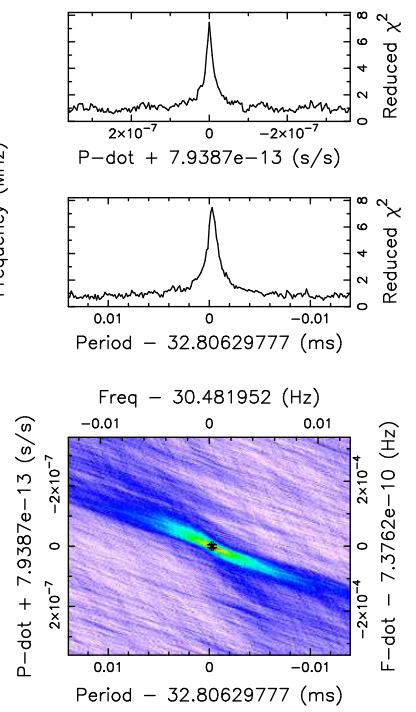

Figure 2.3: Example of the search output diagnostic plot for the newly discovered pulsar PSR J2222-0137. 


\subsection{Timing Observations and Analysis}

Timing observations began in the northern Summer of 2008. At that stage, the nine new pulsars that had been discovered were observed. The first session was conducted at $350 \mathrm{MHz}$ with the GBT using the Spigot backend with $50 \mathrm{MHz}$ of bandwidth and $81.92 \mu$ s sampling time. With observations spread across six hours, this session was used to establish a very accurate period and to grid the pulsars.

Gridding is a method of observing a pulsar at multiple locations to determine a more precise position (Morris et al. 2002). A good estimate in right ascension (RAJ) can be obtained from the discovery plot by looking for the point of inflection of the $\chi^{2}$ versus time graph and finding the corresponding RAJ or looking for the maximum intensity in the plot of phase versus observation time (see Figure 2.3 for example). The $\chi^{2}$ value is a measure of the profile's significance. Each pulsar was observed at the discovery position and four other positions in the cardinal directions for the same amount of time. Each cardinal position was offset by $15^{\prime}$ from the discovery position. A folded profile was made from each data set and along with each observation's RAJ and Declination (DecJ) were used for determining the most likely position of the pulsar. The weighted means for RAJ and DecJ were computed using the reduced $\chi^{2}$ for each profile (see, e.g., Lorimer \& Kramer 2005). If a pulsar was undetected in an observation, it was not used in the weighted mean. The positions given from the gridding are then used for the next observation and also as the beginning position for pulsar timing with $\mathrm{TEMPO}^{4}$.

\footnotetext{
${ }^{4}$ http://www.atnf.csiro.au/research/pulsar/tempo/
} 


\begin{tabular}{lcccc}
\hline $\begin{array}{l}\text { Pulsar } \\
\text { PSR }\end{array}$ & $\begin{array}{c}\text { RAJ } \\
\text { HH:MM:SS }\end{array}$ & $\begin{array}{c}\text { DecJ } \\
\text { DD:MM }\end{array}$ & $\begin{array}{c}\Delta \mathrm{P} \text { discovery } \\
\text { arcmin }\end{array}$ & $\begin{array}{c}\Delta \mathrm{P} \text { gridding } \\
\text { arcmin }\end{array}$ \\
\hline J1555-0515 & $15: 55: 38(5)$ & $-05: 07(1)$ & 12.5 & 8.5 \\
J1612+2008 & $16: 12: 31(8)$ & $+20: 05(2)$ & 8.0 & 3.2 \\
J1735-0243 & $17: 35: 55(4)$ & $-02: 41(1)$ & 4.1 & 4.0 \\
J1737-0814 & $17: 37: 39(4)$ & $-08: 15(1)$ & 4.2 & 2.0 \\
J1903-0848 & $19: 03: 22(5)$ & $-08: 49(1)$ & 2.9 & 2.8 \\
J2012-2029 & $20: 12: 52(9)$ & $-20: 18(1)$ & 21.0 & 11.6 \\
J2033-1938 & $20: 33: 52(4)$ & $-19: 39(1)$ & 1.5 & 1.2 \\
J2111+2108 & $21: 11: 28(5)$ & $+21: 08(2)$ & 8.2 & 2.4 \\
\hline
\end{tabular}

Table 2.3: Post-gridding best-fit positions of pulsars. $\Delta \mathrm{P}$ is the difference between the true position and the discovery and post-gridding positions.

Table 2.3 shows the pulsars' best-fit position from gridding and the difference between the pre- and post-gridding positions with respect to the true position (i.e. that from the final timing solution). The results vary, but all pulsars showed an improved position with respect to the final timing position, with pulsars having a larger initial offset showing greater improvement. The improvement in position offers three benefits: (i) a position closer to the true position lessens the time to get a timing solution; (ii) it moves the pulsar closer to the center of the beam of the telescope, improving the $\mathrm{S} / \mathrm{N}$ and yielding smaller errors on the times of arrivals (TOAs); and (iii) it allows higher frequency observations to occur before a full timing solution is known.

Most of the rest of the follow-up timing observations in the first year (July 2008 - May 2009) were conducted using the Spigot backend at a central frequency of $820 \mathrm{MHz}$ with 1024 channels, $50 \mathrm{MHz}$ of bandwidth and $81.92 \mu$ s sampling time (see Table 2.4 for a complete list of observations). All further observations (June 2009 onwards) were conducted using the newly commissioned Green Bank Ultimate 
Pulsar Processing Instrument (GUPPI) backend (Ransom et al. 2009). Most of the observations were taken at a central frequency of $820 \mathrm{MHz}$ with $200 \mathrm{MHz}$ of bandwidth, 2048 channels, and $40.96 \mu$ s sampling time. For pulsars that had satisfactory timing solutions, the data were taken in on-line folding mode with ten-second integrations, while the rest were taken in search mode the data being taken in psrfits format (Hotan et al. 2004).

TOAs for Spigot and GUPPI search mode data were measured using PRESTO. Each data set was first subbanded and de-dispersed at the pulsar's dispersion measure (DM) into broader frequency channels. We used 16 subbands for long period pulsars and 32 subbands for MSPs. The DM value used for subbanding is either the discovery value or a timing-derived value, if multi-frequency TOAs were available for fitting the DM. The on-line folded GUPPI data require no subbanding.

Folded pulsar profiles were created from the subbanded data using PRESTO; an example output plot is shown in Figure 2.3. These folds were done using a TEMPO parameter file and profiles were folded using 128 bins for normal period pulsars or 64 bins for MSPs. On-line folded GUPPI data were scrunched in time and frequency to form a profile using the PSRCHIVE 5 package with 256 pulse phase bins.

TOAs are measured from the folded profiles using the frequency domain algorithm in PRESTO for all search mode data (Taylor 1992). The TOAs are produced in the Princeton format, with one TOA being produced per observation for each isolated pulsar and three per observation for each binary pulsar for easier deter-

\footnotetext{
${ }^{5}$ http://psrchive.sourceforge.net/current
} 


\begin{tabular}{|c|c|c|c|c|c|}
\hline $\begin{array}{l}\text { MJD range } \\
\text { (days) }\end{array}$ & Machine & $\begin{array}{c}\text { Center Frequency } \\
(\mathrm{MHz})\end{array}$ & $\begin{array}{l}\text { Bandwidth } \\
\text { (MHz) }\end{array}$ & Channels & $\begin{array}{l}\text { Sampling Time } \\
(\mu \mathrm{s})\end{array}$ \\
\hline $54634-54635$ & Spigot & 350 & 50 & 1024 & 81.92 \\
\hline $54644-54726$ & Spigot & 820 & 50 & 1024 & 81.92 \\
\hline 54741 & Spigot & 990 & 50 & 1024 & 81.92 \\
\hline $54767-54856$ & Spigot & 820 & 50 & 1024 & 81.92 \\
\hline 54866 & Spigot & 1500 & 800 & 1024 & 81.92 \\
\hline 54885 & Spigot & 820 & 50 & 1024 & 81.92 \\
\hline 54902-54906 & Spigot & 1500 & 800 & 1024 & 81.92 \\
\hline 54917 & Spigot & 820 & 50 & 1024 & 81.92 \\
\hline 54935 & Spigot & 1500 & 800 & 1024 & 81.92 \\
\hline 54947 & Spigot & 820 & 50 & 1024 & 81.92 \\
\hline 54964 & Spigot & 2000 & 800 & 1024 & 81.92 \\
\hline 54976 & Spigot & 820 & 50 & 1024 & 81.92 \\
\hline 54985 & GUPPI & 1420 & 200 & 2048 & 61.44 \\
\hline 54988-54996 & GUPPI & 1500 & 800 & 2048 & 61.44 \\
\hline 54997 & GUPPI & 2000 & 800 & 2048 & 40.96 \\
\hline $55005-55011$ & GUPPI & 820 & 200 & 2048 & 61.44 \\
\hline 55015 & GUPPI & 2000 & 800 & 2048 & 81.92 \\
\hline $55016-55017$ & GUPPI & 820 & 200 & 2048 & 40.96 \\
\hline 55023 & GUPPI & 350 & 100 & 2048 & 122.88 \\
\hline $55023-55024$ & GUPPI & 350 & 100 & 2048 & 81.92 \\
\hline 55025 & GUPPI & 350 & 100 & 2048 & 122.88 \\
\hline 55026 & GUPPI & 350 & 100 & 2048 & 81.92 \\
\hline $55033-55069$ & GUPPI & 820 & 200 & 2048 & 40.96 \\
\hline 55079 & GUPPI & 350 & 100 & 2048 & 122.88 \\
\hline $55093-55095$ & GUPPI & 820 & 200 & 2048 & 40.96 \\
\hline 55112 & GUPPI & 2000 & 800 & 2048 & 40.96 \\
\hline $55121-55339$ & GUPPI & 820 & 200 & 2048 & 40.96 \\
\hline 55354 & GUPPI & 1400 & 800 & 2048 & 40.96 \\
\hline $55359-55396$ & GUPPI & 820 & 200 & 2048 & 40.96 \\
\hline 55406 & GUPPI & 350 & 100 & 2048 & 81.92 \\
\hline $55453-55639$ & GUPPI & 820 & 200 & 2048 & 40.96 \\
\hline
\end{tabular}

Table 2.4: Observational parameters for timing observations. 
mination of binary parameters. TOAs for the on-line folded data are created with PSRCHIVE and also used the Taylor (1992) method with the same numeration as the search mode data. Templates are created by fitting Gaussian functions to the summed pulse profile. From these Gaussian components, noise-free templates are created for each observing frequency.

An ephemeris file is created for each pulsar for use with TEMPO. These files include astrometric, spin, and if applicable, binary parameters. The period is taken from the discovery plot for isolated pulsars. Binary pulsar periods change with orbital phase and their periods require a more complicated process that will be described in the next paragraph along with determining their binary parameters. Positions in RAJ and DecJ are obtained from gridding of the initial discovery position. All pulsars were assumed to have a $\dot{P}$ value of zero at the start of timing but are measured for when the data span was long enough. The epoch of the period (PEPOCH) was initially taken to be the MJD of the first timing observation and can be adjusted as additional observations are added.

Binary pulsars require more input parameters than isolated pulsars. Initial estimates of the binary parameters are made by fitting a sinusoid to data of barycentric period versus time. Parameters acquired from this fit include the pulsar's barycentric period, orbital period, projection of semi-major axis, and the epoch of passage of periastron.

For each pulsar, the TOAs and ephemeris are used in TEMPO to carry out a standard pulse timing analysis. In this iterative process (for details, see, e.g., Lorimer \& Kramer 2005), a model is generated which keeps track of each neutron 
star rotation over the entire baseline of the observations. The result is a model for the behavior of the pulsar with the root-mean-square (RMS) of the difference between the model and the TOAs. The timing parameters for each pulsar are listed in Tables 2.7 through 2.13, with typically RMS dispersions of a few milliperiods.

\subsection{Other measured parameters}

Along with timing parameters, the follow-up observations can provide other information about the pulsar. The following section discusses other measured properties obtained during the course of these observations.

\subsubsection{0-MHz Fluxes}

Fluxes were measured using the calibration routine associated with PSRCHIVE with the on-line folded GUPPI data. On and off source scans of the extragalactic radio source $3 \mathrm{C} 190$ were used for the flux calibration. Before each observation, a one minute cal-scan was taken with a $25 \mathrm{~Hz}$ noise diode at the receiver. RFI was

removed from the pulsar and cal-scan data using the psrzap utility created by Paul Demorest.

Next, a database is created containing the information for each of the following: on and off flux scans, cal-scan, and pulsar data. Using the file in the database, an absolute flux conversion factor is calculated and applied to the pulsar data. Afterwards a rotation measure fit is always applied to the data, (this procedure will be described in section 2.5.2). Lastly, a mean flux density measurement of total 
intensity is taken from the calibrated data.

\subsubsection{Rotation Measures}

For pulsars with enough linearly polarized flux, rotation measure (RM) values were calculated. This includes 10 of the 13 pulsars in this sample. PSRCHIVE was used with on-line folded GUPPI data for producing the RMs. The method used here is similar to that presented in recent work of Yan et al. (2011).

After the data were calibrated, we test many different RM values from -1000 $-1000 \mathrm{rad} \mathrm{m}^{-2}$ to find the one which provides the most polarized flux. An example plot showing the results of this procedure for PSR J1941+0121 can be seen in Figure 2.4. A Gaussian is fit to the plot of polarized flux versus RM and the midpoint of the Gaussian is taken as the best RM value. A RM correction is applied to the calibrated data before a flux is calculated. The RM values quoted for each pulsar are average values over multiple observations with the standard deviation as the error. This is done in case the ionosphere contaminated any RM values (see, e.g., Yan et al. 2011).

\subsubsection{Pulse Widths at $820 \mathrm{MHz}$}

Pulse widths at $50 \%$ peak intensity, $W_{50}$, are derived either by fitting a Gaussian for single peak profiles or by measuring the width across the outer edge of the profile at $50 \%$ of the peak flux for mulitple component profiles. This is done for each epoch. The $W_{50}$ values for each epoch were averaged and the standard deviation 


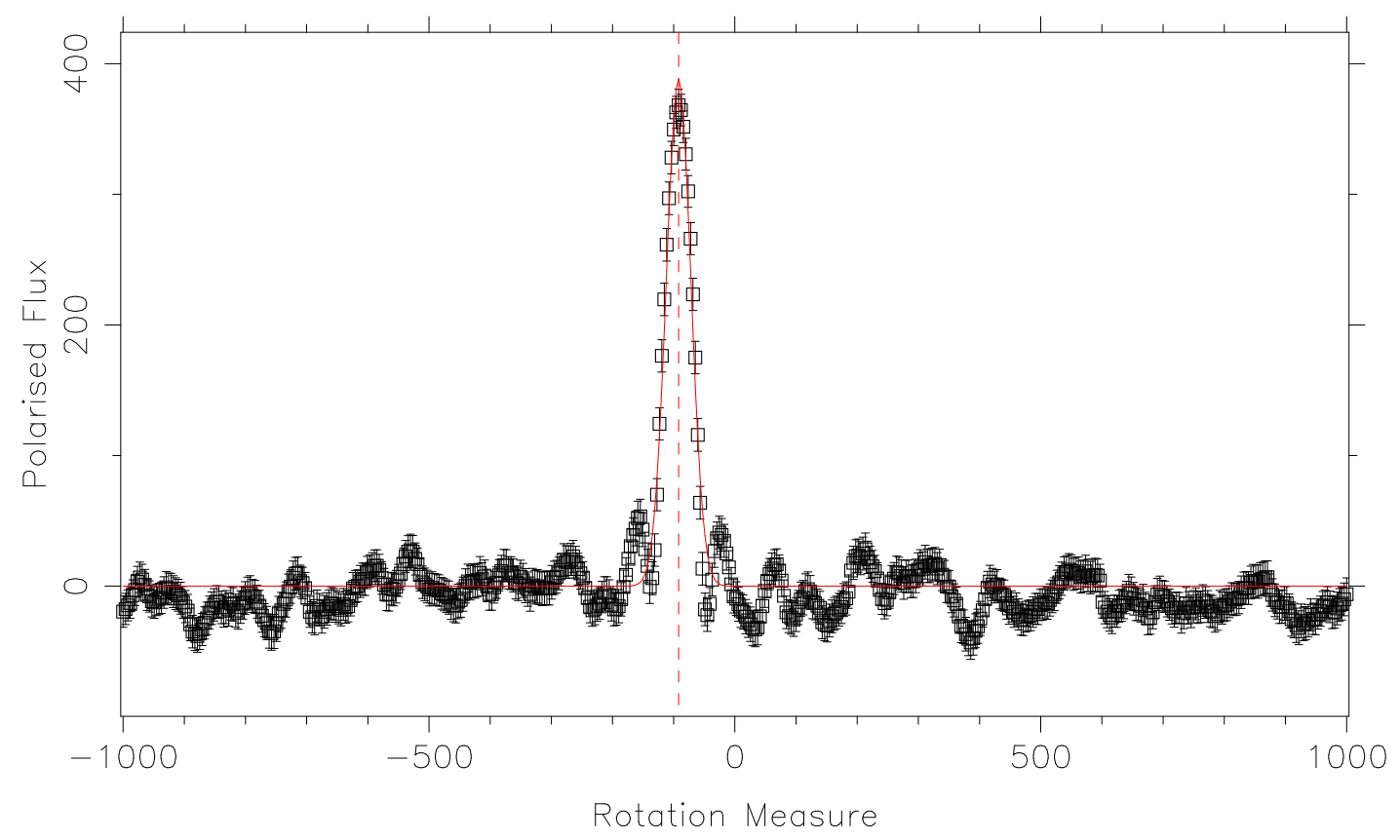

Figure 2.4: Example fit for RM for PSR J1941+0121. Square boxes are the measured values while the dotted line is the best fit value.

was taken as the error.

\subsection{Discussion}

Results from pulsar timing and the analysis mentioned in Section 2.5 are listed in Tables 2.7 through 2.13 for each individual pulsar and a plot showing the location of each new pulsar on the $P-\dot{P}$ diagram is shown in Figure 2.5. All properties are quoted with $1 \sigma$ errors and the DM-derived distances are from the NE2001 electron model (Cordes \& Lazio 2002). The distances have errors $\sim 20 \%$. The equations for calculating surface magnetic field strength, characteristic age, and spin-down luminosity are given in Chapter 1. In the following sections properties of individual pulsars that may have additional applications beyond the results of this work will be discussed. 


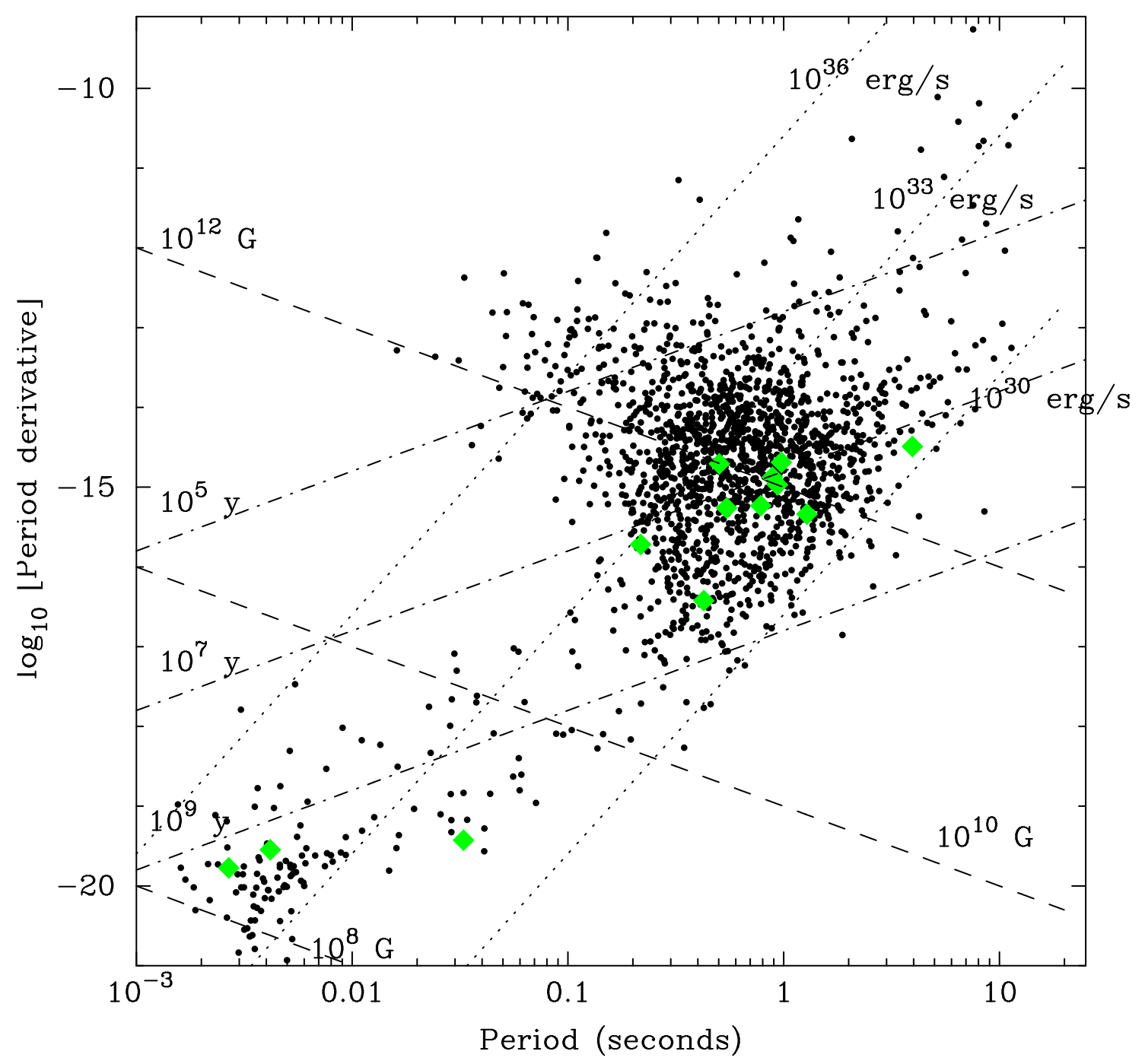

Figure 2.5: $P-\dot{P}$ diagram showing the new discoveries as green diamonds. The pulsar population is taken for the ATNF pulsar catalog (Manchester et al. 2005). 


\subsubsection{PSR J1327-0755}

At the time of discovery, the narrow $350-\mathrm{MHz}$ pulse width of $0.25 \mathrm{~ms}$ and location well off the Galactic plane $\left(b=54.3^{\circ}\right)$ of PSR J1327-0755 were encouraging signs that this pulsar may be a good addition to PTAs for the search for gravitational waves (Foster \& Backer 1990). However, analysis at $820 \mathrm{MHz}$ indicates that PSR J1327-0755 may not meet the standards needed for a PTA pulsar due to variations in its flux from interstellar scintillation. While 95\% of GUPPI observations resulted in a detection, only $\sim 35 \%$ of Spigot observations resulted in a detection. The pulsar was detectable in only $\sim 25 \%$ of the bandpass, most likely the result of interstellar scintillation. The large difference in S/N between 350 and $820 \mathrm{MHz}$ implies that PSR J1327-0755 has a steep spectral index but scintillation has hindered any attempt to reliably measure this. With an orbital period of 8.44 days and a projected semi-major axis of 6.65 lt-s, PSR J1327-0755 has a minimum companion mass of $0.22 M_{\odot}$ and with its location on the $P-\dot{P}$ diagram, its companion is most likely a white dwarf.

\subsubsection{PSR J1623-0841}

PSR J1623-0841 was discovered in only the single-pulse search and was labeled a rotating radio transient (RRAT). Later observations at $350 \mathrm{MHz}$ with Spigot showed single pulses but no detections were made at $820 \mathrm{MHz}$. Gridding observations were unable to localize the position better than the discovery position because of its transient nature. The final TEMPO position was $20^{\prime}$ away from the discovery 
position (outside the GBT's $820-\mathrm{MHz}$ beam) and a timing solution was only attainable with the increased sensitivity of GUPPI and a dense set of observations at 350 MHz. A full discussion of PSR J1623-0841 will be presented in Chapter 3.

\subsubsection{PSR J1737-0814}

PSR J1737-0814 is a binary pulsar in an eccentric orbit $(e=0.013)$ with an orbital period of 79.38 days and a minimum mass companion of $0.06 M_{\odot}$. Phinney (1992) predicts a relationship between orbital period and eccentricity of

$$
<e^{2}>^{0.5}=1.5 \times 10^{-4}\left(P_{b} / 100 \text { days }\right) \text { for } P_{b}>P_{\text {crit }} \approx 25 \text { days }
$$

The eccentricity of PSR J1737-0814 is about two orders of magnitude greater than what is predicted by this relationship and is the second largest eccentricity for a Galactic plane MSP. This large deviation is possible though unusual for Galactic field MSPs; PSR J1903+0327 is a binary pulsar with a orbital period of 95.17 days and an eccentricity of 0.437 , which may have evolved from a triple system (Freire et al. 2011).

If we assume that the companion of PSR J1737-0814 is a white dwarf, which is very common for pulsars located in that area of the $P-\dot{P}$ diagram, and the minimum mass of the white dwarf is $0.6 M_{\odot}$, then we can obtain a maximum inclination angle of 8 degrees assuming a pulsar mass of $1.35 M_{\odot}$. With the wide pulse width (see Figure 2.9) and low inclination angle, a measure of the companion's mass through the Shapiro delay (Shapiro 1964) would be impossible. Other Post-Keplerian parameters 
such as the relativistic advance of periastron are also unmeasurable due to the low precision of the TOAs provided by this pulsar.

\subsubsection{PSR J1941+0121}

PSR J1941+0121 has the shortest period (217 ms) of the non-recycled pulsars found in this survey and about $85 \%$ of its total flux is linearly polarized. This high percentage of linearly polarized flux along with its relatively wide pulse width $(\epsilon=0.093)$ has allowed us to produce a model of the pulsar's emission using the PSRCHIVE software package. A complex-value Rotating Vector Model (RVM; Radhakrishnan \& Cooke 1969) was fit to the Stokes Q and Stokes U profiles, treating them as real and imaginary numbers instead of the single parameter value of position angle. Figure 2.6 shows the fit of the model and Table 2.5 gives the model fit parameters. The model gives an impact parameter $(\beta=\zeta-\alpha)$ of $11^{\circ}$ and an inclination angle of $116^{\circ}$. Due to the large error of the impact parameter $\left(13^{\circ}\right)$, we are unable to determine if we see an inner or outer line of sight. The model has a $\chi^{2}$ of 37.8 with 37 degrees of freedom (on-pulse phase bins), giving a reduced $\chi^{2}$ of 1.02 .

\subsubsection{PSR J2222-0137}

PSR J2222-0137 is in a low eccentricity orbit $(e=0.00038)$ with an orbital period of 2.4 days and a projected semi-major axis of 10.8 lt-s. The orbital parameters lead to a mass function value of $0.229 M_{\odot}$. Assuming a pulsar mass of 


\begin{tabular}{lcc}
\hline Parameter & $\begin{array}{c}\text { Angle } \\
\text { (degrees) }\end{array}$ & $\begin{array}{c}\text { Error } \\
\text { (degrees) }\end{array}$ \\
\hline$\psi_{0}$ & -82 & 2 \\
$\zeta$ & 127 & 9 \\
$\alpha$ & 116 & 10 \\
$\phi_{0}$ & 172.3 & 0.5 \\
\hline
\end{tabular}

Table 2.5: RVM model fit results for PSR J1941+0121. Here $\phi_{0}$ is the pulse phase of magnetic meridian, $\psi_{0}$ is position angle at $\phi_{0}, \alpha$ is the colatitude of magnetic axis, and $\zeta$ is the colatitude of the observer's line of sight.

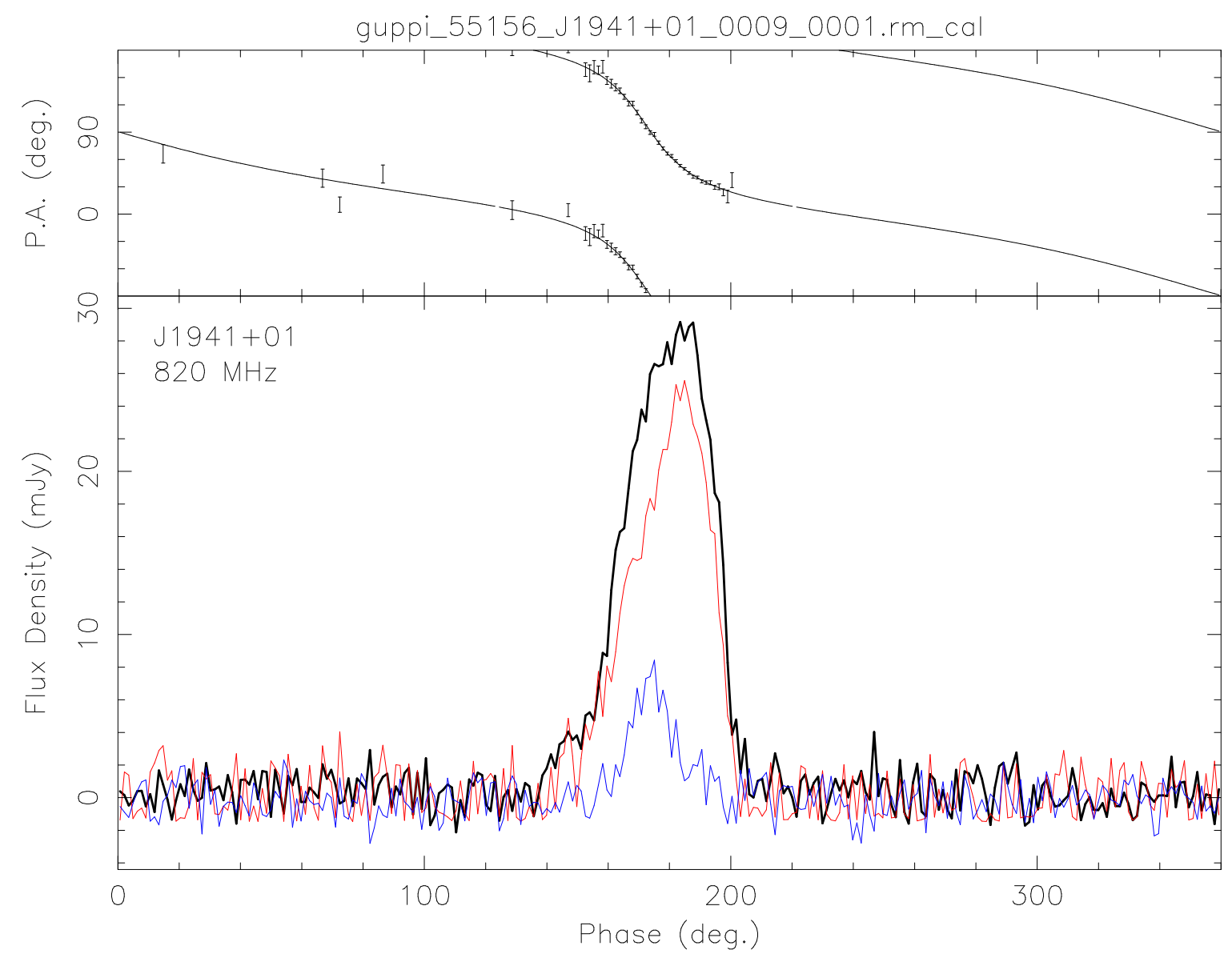

Figure 2.6: RVM model fit for the radio emission of PSR J1941+0121. The top plot show parallactic angle versus pulse phase, while the bottom plot shows integrated pulse flux density versus pulse phase for total intensity (black line), linearly polarized flux (red line), and circularly polarized flux (blue line). 
$1.35 M_{\odot}$ this gives us a minimum companion mass of $1.11 M_{\odot}$. The spin period of $33 \mathrm{~ms}$, the binary parameters, and the lack of a significant $\dot{P}$ indicate this pulsar is a partially recycled pulsar. Based on these parameters, and from what is known about other binary pulsars, the companion star is most likely a member of the so-called "intermediate-mass binary pulsars" (e.g. Camilo et al. 2001) in which the companion star is a $\mathrm{CO}$ white dwarf. It is also possible, though less likely, given the low orbital eccentricity, that PSR J2222-0137 is a member of a double neutron star binary system. Many follow-up observations of PSR J2222-0137 at multiple frequencies have been searched for a second pulsar without success with a $\mathrm{S}_{\min }$, minimum detectable luminosity, of $11 \mu \mathrm{Jy}$ at $1400 \mathrm{MHz}$ which gives a probability of detection of $98 \%$ assuming a distance of 300 pc using the Faucher-Giguere, C. A. \& Kaspi (2006) luminosity model.

A minimum companion mass of $1.11 M_{\odot}$ and timing residuals of $\sim 16 \mu$ s for PSR J2222-0137 suggest that the companion mass and orbital inclination could be measured by the Shapiro delay as the pulsar's emission passes through the gravitational potential of the companion. In order to investigate the feasibility of observing this effect, simulations have been done using TEMPO $2^{6}$ for different scenarios that will satisfy the mass function and are shown in Figure 2.7. These simulations assumed a TOA error of $3.5 \mu$ s and two-minute integration times. The observations were placed at each extrema in the Shapiro delay higher harmonics, with a sixhour observation at superior conjunction and two-hour observations at each other extrema. This simulated observing strategy provides detection limits only $20 \%$ less

\footnotetext{
${ }^{6}$ www.atnf.csiro.au/research/pulsar/tempo2/
} 
significant than observing a whole orbit with the same TOA cadence, but using only one-fifth of the time.

Using the DM value of $3.28 \mathrm{pc} \mathrm{cm}^{-3}$, the NE2001 electron density model predicts a distance of $~ 300$ pc to PSR J2222-0137 (Cordes \& Lazio 2002). Assuming this distance estimate is reliable it implies PSR J2222-0137 is the second closest binary pulsar system after PSR J0437-4715 at a DM of $2.64 \mathrm{pc} \mathrm{cm}^{-3}$ and a distance of 156 pc (Deller et al. 2009). For pulsars located this close to us, a large window of multi-wavelength observations opens up. These include VLBA observations to measure proper motion and parallax, optical observations to search for an optical companion, X-ray observations to look for blackbody emission from the neutron star, and gamma-ray observations to look at higher magnetosphere emission. All the observations proposed in this paragraph and the Shapiro delay observations have occurred, except the last epochs of the VLBA observations, and the results will be reported in future publications.

\subsection{Conclusions and Future Work}

The 350-MHz drift-scan revealed many new interesting pulsars to study across the whole electromagnetic spectrum. Along with PSR J2222-0137, featured in this work, PSR J1023+0038 (Archibald et al. 2009), PSR J2256-1023 (Stairs et al. in prep), and PSR J0348+0438 (Lynch et al. in prep) have all had multi-messenger studies conducted with them. This survey was the pilot for a new survey called the Green Bank North Celestrial Cap (GBNCC) survey. In this survey we are taking 


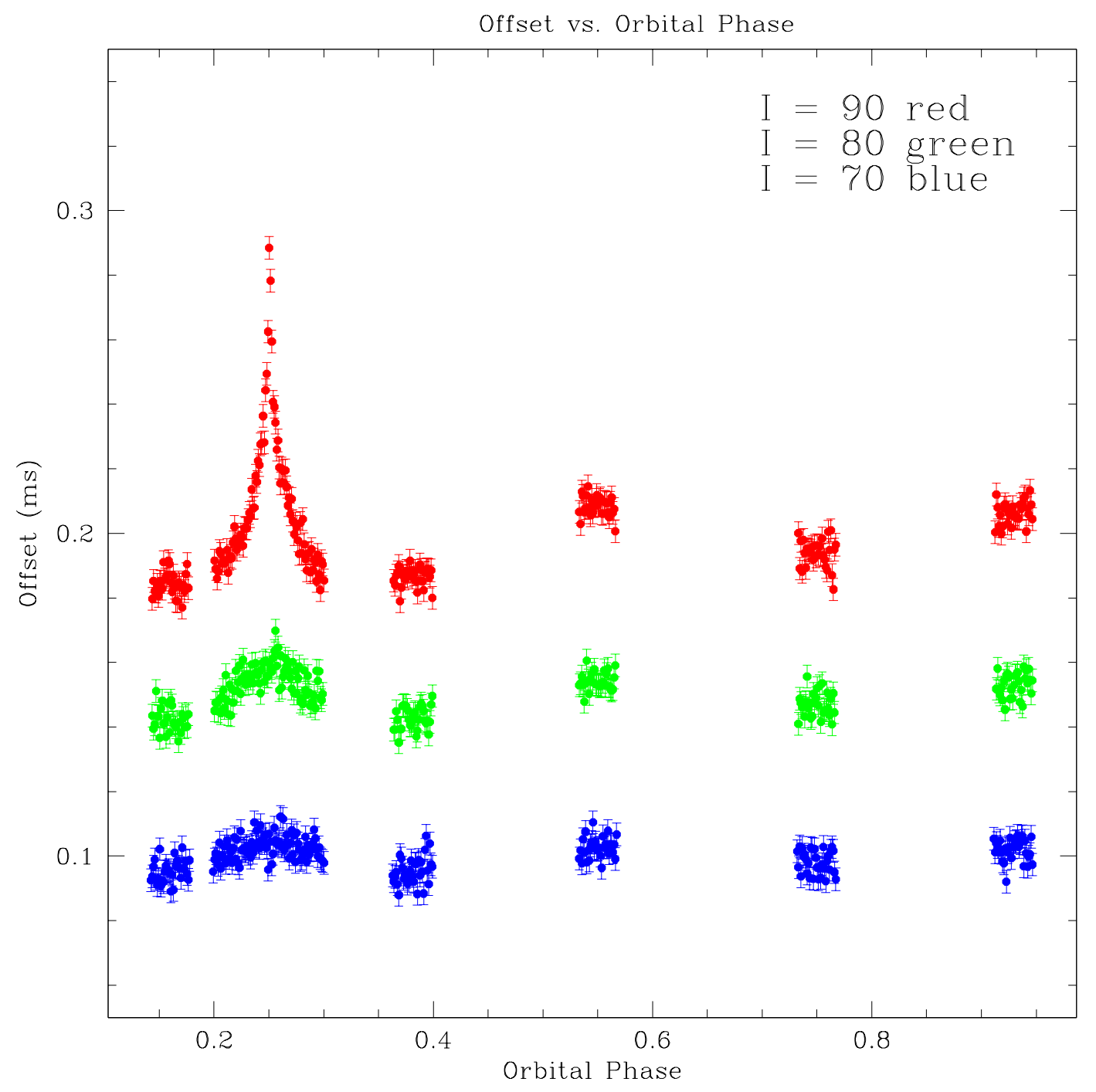

Figure 2.7: TEMPO2 simulations showing the Shapiro delay expected for PSR J2222-0137 for multiple inclination angles. The x-axis is the orbital phase of the pulsar with respect to periastron and the y-axis is the excess time delay caused by the companion as the pulsar's signal passes through the warped space-time around the companion. From top to bottom: $\mathrm{i}=90^{\circ}$ (red), $\mathrm{i}=80^{\circ}$, (green), and $\mathrm{i}=70^{\circ}$ (blue). 


\begin{tabular}{|c|c|c|}
\hline Parameter & PSR J1555-0515 & PSR J1612+2008 \\
\hline \multicolumn{3}{|l|}{ Timing Parameters } \\
\hline Right Ascension (J2000) & $15: 55: 40.102(8)$ & $16: 12: 23.429(3)$ \\
\hline Declination (J2000) & $-05: 15: 56.8(3)$ & $20: 08: 18.37(5)$ \\
\hline Spin Period (s) & $0.975409891127(7)$ & $0.4266459810255(16)$ \\
\hline Period Derivative $\left(\mathrm{s} \mathrm{s}^{-1}\right)$ & $2.0519(8) \times 10^{-15}$ & $3.758(17) \times 10^{-17}$ \\
\hline Dispersion Measure $\left(\mathrm{pc} \mathrm{cm}^{-3}\right)$ & $23.489(14)$ & $19.539(6)$ \\
\hline Reference Epoch (MJD) & 54985 & 54985 \\
\hline Span of Timing Data & $54635-55335$ & $54635-55302$ \\
\hline Number of TOAs & 36 & 40 \\
\hline RMS Residual ( $\mu \mathrm{s})$ & 640 & 350 \\
\hline \multicolumn{3}{|l|}{ Derived Parameters } \\
\hline Galactic Longitude (degrees) & 3.97 & 35.52 \\
\hline Galactic Latitude (degrees) & 34.97 & 43.74 \\
\hline Distance $(\mathrm{kpc})$ & 1.2 & 1.3 \\
\hline Distance Off Plane (kpc) & 0.69 & 0.90 \\
\hline Surface Magnetic Field (10 12 Gauss) & 1.41 & 0.126 \\
\hline Spin-down Luminosity $\left(10^{31} \mathrm{ergs} \mathrm{s}^{-1}\right)$ & 2.75 & 0.603 \\
\hline Characteristic Age (Myr) & 7.41 & 178 \\
\hline 820 MHz Flux Density (mJy) & $1.2(2)$ & $0.83(11)$ \\
\hline $\mathrm{W}_{50} 820 \mathrm{MHz}(\mathrm{ms})$ & $12.9(3)$ & $3.29(10)$ \\
\hline Rotation Measure $\left(\mathrm{rad} \mathrm{m}{ }^{-2}\right)$ & $1.3(3.0)$ & $22(3)$ \\
\hline
\end{tabular}

Table 2.6: Timing and derived parameters for newly discovered isolated pulsars.

advantage of the newest pulsar instrument on the GBT, the GUPPI backend. This survey is conducted with real 8 bit sampling, 4096 frequency channels, $100 \mathrm{MHz}$ of bandwidth, 120 second integration times, and $81.92 \mu$ s sampling time at a center frequency of $350 \mathrm{MHz}$. This survey will be about a factor of two more sensitive than the survey described in this paper and will be a powerful probe of the millisecond pulsar population in the northern sky. The GBNCC survey will cover the whole entire northern sky visible by the GBT. 


\begin{tabular}{|c|c|c|}
\hline Parameter & PSR J1623-0841 & PSR J1633-2009 \\
\hline \multicolumn{3}{|l|}{ Timing Parameters } \\
\hline Right Ascension (J2000) & $16: 23: 42.711(9)$ & $16: 33: 55.34(6)$ \\
\hline Declination (J2000) & $-08: 41: 36.3(5)$ & $-20: 09: 59(5)$ \\
\hline Spin Period (s) & $0.503014992514(6)$ & $0.935557045033(17)$ \\
\hline Period Derivative $\left(\mathrm{s} \mathrm{s}^{-1}\right)$ & $1.958(3) \times 10^{-15}$ & $1.0680(18) \times 10^{-15}$ \\
\hline Dispersion Measure $\left(\mathrm{pc} \mathrm{cm}^{-3}\right)$ & $60.433(16)$ & $48.20(6)$ \\
\hline Reference Epoch (MJD) & 55048 & 54993 \\
\hline Span of Timing Data & $54635-55522$ & $54651-55335$ \\
\hline Number of TOAs & 36 & 29 \\
\hline RMS Residual $(\mu \mathrm{s})$ & 765 & 1347 \\
\hline \multicolumn{3}{|l|}{ Derived Parameters } \\
\hline Galactic Longitude (degrees) & 5.77 & 357.63 \\
\hline Galactic Latitude (degrees) & 27.37 & 18.33 \\
\hline Distance $(\mathrm{kpc})$ & 3.3 & 1.6 \\
\hline Distance Off Plane (kpc) & 1.5 & 0.5 \\
\hline Surface Magnetic Field (10 ${ }^{12}$ Gauss) & 1.00 & 1.00 \\
\hline Spin-down Luminosity $\left(10^{31} \mathrm{ergs} \mathrm{s}^{-1}\right)$ & 19.5 & 1.62 \\
\hline Characteristic Age (Myr) & 4.07 & 13.8 \\
\hline 820 MHz Flux Density (mJy) & $42(13)$ & $1.35(4)$ \\
\hline $\mathrm{W}_{50} 820 \mathrm{MHz}(\mathrm{ms})$ & $11.2(3)$ & $32.2(1.0)$ \\
\hline Rotation Measure $\left(\mathrm{rad} \mathrm{m} \mathrm{m}^{-2}\right)$ & $\mathrm{N} / \mathrm{A}$ & $-0.1(1.2)$ \\
\hline
\end{tabular}

Table 2.7: Timing and derived parameters for newly discovered isolated pulsars. 


\begin{tabular}{|c|c|c|}
\hline Parameter & PSR J1735-0243 & PSR J1903-0848 \\
\hline \multicolumn{3}{|l|}{ Timing Parameters } \\
\hline Right Ascension (J2000) & $17: 35: 48.31(9)$ & 19:03:11.264(9) \\
\hline Declination (J2000) & $-02: 43: 51(3)$ & $-08: 48: 56.8(4)$ \\
\hline Spin Period (s) & $0.782886976234(10)$ & $0.887324640598(8)$ \\
\hline Period Derivative $\left(\mathrm{s} \mathrm{s}^{-1}\right)$ & $5.86(6) \times 10^{-16}$ & $1.3302(9) \times 10^{-15}$ \\
\hline Dispersion Measure $\left(\mathrm{pc} \mathrm{cm}^{-3}\right)$ & $55.3(2)$ & $66.987(15)$ \\
\hline Reference Epoch (MJD) & 54827 & 54987 \\
\hline Span of Timing Data & $54352-55302$ & $54634-55339$ \\
\hline Number of TOAs & 39 & 51 \\
\hline RMS Residual $(\mu \mathrm{s})$ & 10585 & 930 \\
\hline \multicolumn{3}{|l|}{ Derived Parameters } \\
\hline Galactic Longitude (degrees) & 21.56 & 26.38 \\
\hline Galactic Latitude (degrees) & 15.45 & -6.60 \\
\hline Distance $(\mathrm{kpc})$ & 1.9 & 2.0 \\
\hline Distance Off Plane (kpc) & 0.51 & 0.23 \\
\hline Surface Magnetic Field (10 12 Gauss) & 0.676 & 1.10 \\
\hline Spin-down Luminosity $\left(10^{31} \mathrm{ergs} \mathrm{s}^{-1}\right)$ & 1.51 & 2.34 \\
\hline Characteristic Age (Myr) & 20.9 & 10.5 \\
\hline 820 MHz Flux Density (mJy) & $1.3(3)$ & $1.4(2)$ \\
\hline $\mathrm{W}_{50} 820 \mathrm{MHz}(\mathrm{ms})$ & $102(2)$ & $12.3(2)$ \\
\hline Rotation Measure $\left(\mathrm{rad} \mathrm{m} \mathrm{m}^{-2}\right)$ & $28.3(1.9)$ & $4.4(9)$ \\
\hline
\end{tabular}

Table 2.8: Timing and derived parameters for newly discovered isolated pulsars. 


\begin{tabular}{|c|c|c|}
\hline Parameter & PSR J1941+0121 & PSR J2012-2029 \\
\hline \multicolumn{3}{|l|}{ Timing Parameters } \\
\hline Right Ascension (J2000) & $19: 41: 16.042(6)$ & $20: 12: 46.8(2)$ \\
\hline Declination (J2000) & $01: 21: 39.5(2)$ & $-20: 29: 42(14)$ \\
\hline Spin Period (s) & $0.217317451824(7)$ & $0.544001870607(8)$ \\
\hline Period Derivative $\left(\mathrm{s} \mathrm{s}^{-1}\right)$ & $1.913(2) \times 10^{-16}$ & $5.477(8) \times 10^{-16}$ \\
\hline Dispersion Measure $\left(\mathrm{pc} \mathrm{cm}^{-3}\right)$ & $52.3(6)$ & $37.42(8)$ \\
\hline Reference Epoch (MJD) & 55026 & 54987 \\
\hline Span of Timing Data & $54712-55339$ & $54635-55339$ \\
\hline Number of TOAs & 33 & 33 \\
\hline RMS Residual $(\mu \mathrm{s})$ & 673 & 1090 \\
\hline \multicolumn{3}{|l|}{ Derived Parameters } \\
\hline Galactic Longitude (degrees) & 39.91 & 22.41 \\
\hline Galactic Latitude (degrees) & -10.43 & -26.70 \\
\hline Distance $(\mathrm{kpc})$ & 2.2 & 1.4 \\
\hline Distance Off Plane (kpc) & 0.40 & 0.63 \\
\hline Surface Magnetic Field (10 12 Gauss) & 0.204 & 0.550 \\
\hline Spin-down Luminosity $\left(10^{31} \operatorname{ergs~s}^{-1}\right)$ & 22.9 & 4.27 \\
\hline Characteristic Age (Myr) & 17.8 & 15.5 \\
\hline $820 \mathrm{MHz}$ Flux Density (mJy) & $1.7(5)$ & $1.00(12)$ \\
\hline $\mathrm{W}_{50} 820 \mathrm{MHz}(\mathrm{ms})$ & $20.2(1.1)$ & $24.2(7)$ \\
\hline Rotation Measure $\left(\mathrm{rad} \mathrm{m} \mathrm{m}^{-2}\right)$ & $-92.0(3)$ & $\mathrm{N} / \mathrm{A}$ \\
\hline
\end{tabular}

Table 2.9: Timing and derived parameters for newly discovered isolated pulsars. 


\begin{tabular}{|c|c|c|}
\hline Parameter & PSR J2033-1938 & PSR J2111+2106 \\
\hline \multicolumn{3}{|l|}{ Timing Parameters } \\
\hline Right Ascension (J2000) & $20: 33: 54.8(2)$ & $21: 11: 33.12(3)$ \\
\hline Declination $(\mathrm{J} 2000)$ & $-19: 38: 27(12)$ & $21: 06: 07.0(7)$ \\
\hline Spin Period (s) & $1.28171902368(2)$ & $3.95385295935(13)$ \\
\hline Period Derivative $\left(\mathrm{s} \mathrm{s}^{-1}\right)$ & $4.58(2) \times 10^{-16}$ & $3.231(13) \times 10^{-15}$ \\
\hline Dispersion Measure $\left(\mathrm{pc} \mathrm{cm}^{-3}\right)$ & $23.47(3)$ & $59.77(6)$ \\
\hline Reference Epoch (MJD) & 54987 & 54987 \\
\hline Span of Timing Data & $54635-55339$ & $54635-55339$ \\
\hline Number of TOAs & 37 & 35 \\
\hline RMS Residual $(\mu \mathrm{s})$ & 1533 & 2686 \\
\hline \multicolumn{3}{|l|}{ Derived Parameters } \\
\hline Galactic Longitude (degrees) & 25.32 & 69.40 \\
\hline Galactic Latitude (degrees) & -31.04 & -18.20 \\
\hline Distance $(\mathrm{kpc})$ & 1.00 & 3.8 \\
\hline Distance Off Plane (kpc) & 0.51 & 1.2 \\
\hline Surface Magnetic Field (10 12 Gauss) & 0.759 & 3.55 \\
\hline Spin-down Luminosity $\left(10^{31} \mathrm{ergs} \mathrm{s}^{-1}\right)$ & 0.269 & 0.0646 \\
\hline Characteristic Age (Myr) & 43.6 & 19.1 \\
\hline $820 \mathrm{MHz}$ Flux Density (mJy) & $1.13(15)$ & $1.08(10)$ \\
\hline $\mathrm{W}_{50} 820 \mathrm{MHz}(\mathrm{ms})$ & $32.4(3)$ & $45(2)$ \\
\hline Rotation Measure $\left(\mathrm{rad} \mathrm{m} \mathrm{m}^{-2}\right)$ & $-17.7(5)$ & $-75.3(8)$ \\
\hline
\end{tabular}

Table 2.10: Timing and derived parameters for newly discovered isolated pulsars. 


\begin{tabular}{|c|c|c|}
\hline Parameter & PSR J1327-0755 & PSR J1737-0814 \\
\hline \multicolumn{3}{|c|}{ Timing Parameters } \\
\hline Right Ascension (J2000) & $13: 27: 57.984(10)$ & $17: 37: 33.31(9)$ \\
\hline Declination (J2000) & $-07: 55: 16.0(4)$ & $-08: 14: 08.42(15)$ \\
\hline Spin Period (s) & $0.002677923197570(2)$ & $0.00417501702755(13)$ \\
\hline Period Derivative $\left(\mathrm{s} \mathrm{s}^{-1}\right)$ & $1.02(3) \times 10^{-20}$ & $4.34(17) \times 10^{-20}$ \\
\hline Dispersion Measure $\left(\mathrm{pc} \mathrm{cm}^{-3}\right)$ & $27.9153(9)$ & $55.349(8)$ \\
\hline Reference Epoch (MJD) & 55112 & 55007 \\
\hline Span of Timing Data & $54651-55575$ & $54651-55575$ \\
\hline Number of TOAs ${ }^{a}$ & 147 & 150 \\
\hline RMS Residual $(\mu \mathrm{s})$ & 38 & 81 \\
\hline \multicolumn{3}{|c|}{ Binary Parameters } \\
\hline Binary Model & ELL1 & $\mathrm{BT}$ \\
\hline Orbital Period (days) & $8.43908560(12)$ & $79.37793(2)$ \\
\hline Projected Semi-major Axis (lt-s) & $6.645761(9)$ & $9.456345(15)$ \\
\hline Epoch Of Periastron (MJD) & $54725.46(8)$ & $54629.950(4)$ \\
\hline Orbital Eccentricity & $2.1(2) \times 10^{-5}$ & $1.2249(4) \times 10^{-2}$ \\
\hline Longitude of Periastron (degrees) & $14.383(9)$ & $106.102(17)$ \\
\hline Mass Function $\left(M_{\odot}\right)$ & $0.004425132(16)$ & $0.0001440961(7)$ \\
\hline Minimum Companion $\operatorname{Mass}^{b}\left(M_{\odot}\right)$ & $0.2219572(8)$ & $0.0661123(4)$ \\
\hline \multicolumn{3}{|c|}{ Derived Parameters } \\
\hline Galactic Longitude (degrees) & 318.39 & 16.86 \\
\hline Galactic Latitude (degrees) & 53.85 & 12.34 \\
\hline Distance $(\mathrm{kpc})$ & 1.7 & 1.7 \\
\hline Distance Off Plane (kpc) & 1.4 & 0.36 \\
\hline Surface Magnetic Field ( $10^{12}$ Gauss) & $1.66 \times 10^{-4}$ & $1.27 \times 10^{-4}$ \\
\hline Spin-down Luminosity $\left(10^{31} \mathrm{ergs} \mathrm{s}^{-1}\right)$ & 676 & 741 \\
\hline Characteristic Age (Myr) & 4169 & 1513 \\
\hline $820 \mathrm{MHz}$ Flux Density (mJy) & $0.9(2)$ & $2.7(2)$ \\
\hline $\mathrm{W}_{50} 820 \mathrm{MHz}(\mathrm{ms})$ & $0.131(5)$ & $0.52(6)$ \\
\hline Rotation Measure $\left(\mathrm{rad} \mathrm{m}^{-2}\right)$ & $\mathrm{N} / \mathrm{A}$ & $71.4(1.4)$ \\
\hline
\end{tabular}

${ }^{a}$ Three TOAs produced per observation.

${ }^{b}$ Assumes a pulsar mass of $1.35 M_{\odot}$.

Table 2.11: Timing and derived parameters for newly discovered binary pulsars. 


\begin{tabular}{|c|c|}
\hline Parameter & PSR J2222-0137 \\
\hline \multicolumn{2}{|c|}{ Timing Parameters } \\
\hline Right Ascension (J2000) & $22: 22: 05.9637(7)$ \\
\hline Declination (J2000) & $-01: 37: 15.73(3)$ \\
\hline RA Proper Motion (mas $\mathrm{yr}^{-1}$ ) & $43(3)$ \\
\hline DEC Proper Motion $\left(\right.$ mas $\mathrm{yr}^{-1}$ ) & $<9$ \\
\hline Spin Period (s) & $0.032817859051169(6)$ \\
\hline Period Derivative $\left(\mathrm{s} \mathrm{s}^{-1}\right)$ & $5.82(2) \times 10^{-20}$ \\
\hline Dispersion Measure $\left(\mathrm{pc} \mathrm{cm}^{-3}\right)$ & $3.27765(15)$ \\
\hline Reference Epoch (MJD) & 55369 \\
\hline Span of Timing Data & $55005-55639$ \\
\hline Number of TOAs ${ }^{a}$ & 169 \\
\hline RMS Residual $(\mu \mathrm{s})$ & 16 \\
\hline
\end{tabular}

\begin{tabular}{|c|c|}
\hline \multicolumn{2}{|c|}{ Binary Parameters } \\
\hline Binary Model & ELL1 \\
\hline Orbital Period (days) & $2.4457599952(2)$ \\
\hline Projected Semi-major Axis (lt-s) & $10.8480199(14)$ \\
\hline Epoch Of Periastron (MJD) & $54979.05568(11)$ \\
\hline Orbital Eccentricity & $3.8169(13) \times 10^{-4}$ \\
\hline Longitude of Periastron (degrees) & $119.834(17)$ \\
\hline Mass Function $\left(M_{\odot}\right)$ & $0.22914294(9)$ \\
\hline Minimum Companion $\operatorname{Mass}^{b}\left(M_{\odot}\right)$ & $1.1173504(5)$ \\
\hline \multicolumn{2}{|c|}{ Derived Parameters } \\
\hline Galactic Longitude (degrees) & 62.02 \\
\hline Galactic Latitude (degrees) & -46.08 \\
\hline Distance (kpc) & 0.31 \\
\hline Distance Off Plane (kpc) & 0.22 \\
\hline Transverse Velocity $\left(\mathrm{km} \mathrm{s}^{-1}\right)$ & $<66$ \\
\hline Shklovski Effect $\left(\mathrm{s} \mathrm{s}^{-1}\right)$ & $<5.02 \times 10^{-20}$ \\
\hline Intrinsic Spin-down $\left(\mathrm{s} \mathrm{s}^{-1}\right)$ & $>8.1 \times 10^{-21}$ \\
\hline Surface Magnetic Field ${ }^{c}\left(10^{12}\right.$ Gauss $)$ & 0.00141 \\
\hline Spin-down Luminosity $^{c}\left(10^{31} \operatorname{ergs~s}^{-1}\right)$ & 2.09 \\
\hline 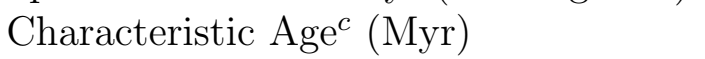 & 8710 \\
\hline 820 MHz Flux Density (mJy) & $2.6(5)$ \\
\hline $\mathrm{W}_{50} 820 \mathrm{MHz}(\mathrm{ms})$ & $0.570(5)$ \\
\hline Rotation Measure $\left(\mathrm{rad} \mathrm{m} \mathrm{m}^{-2}\right)$ & $1.8(6)$ \\
\hline
\end{tabular}

a Three TOAs produced per observation.

${ }^{b}$ Assumes a pulsar mass of $1.35 M_{\odot}$.

${ }^{c}$ Values are not corrected for Shklovskii effect (Shklovskii 1970).

Table 2.12: Timing and derived parameters for newly discovered PSR J2222-0137. 

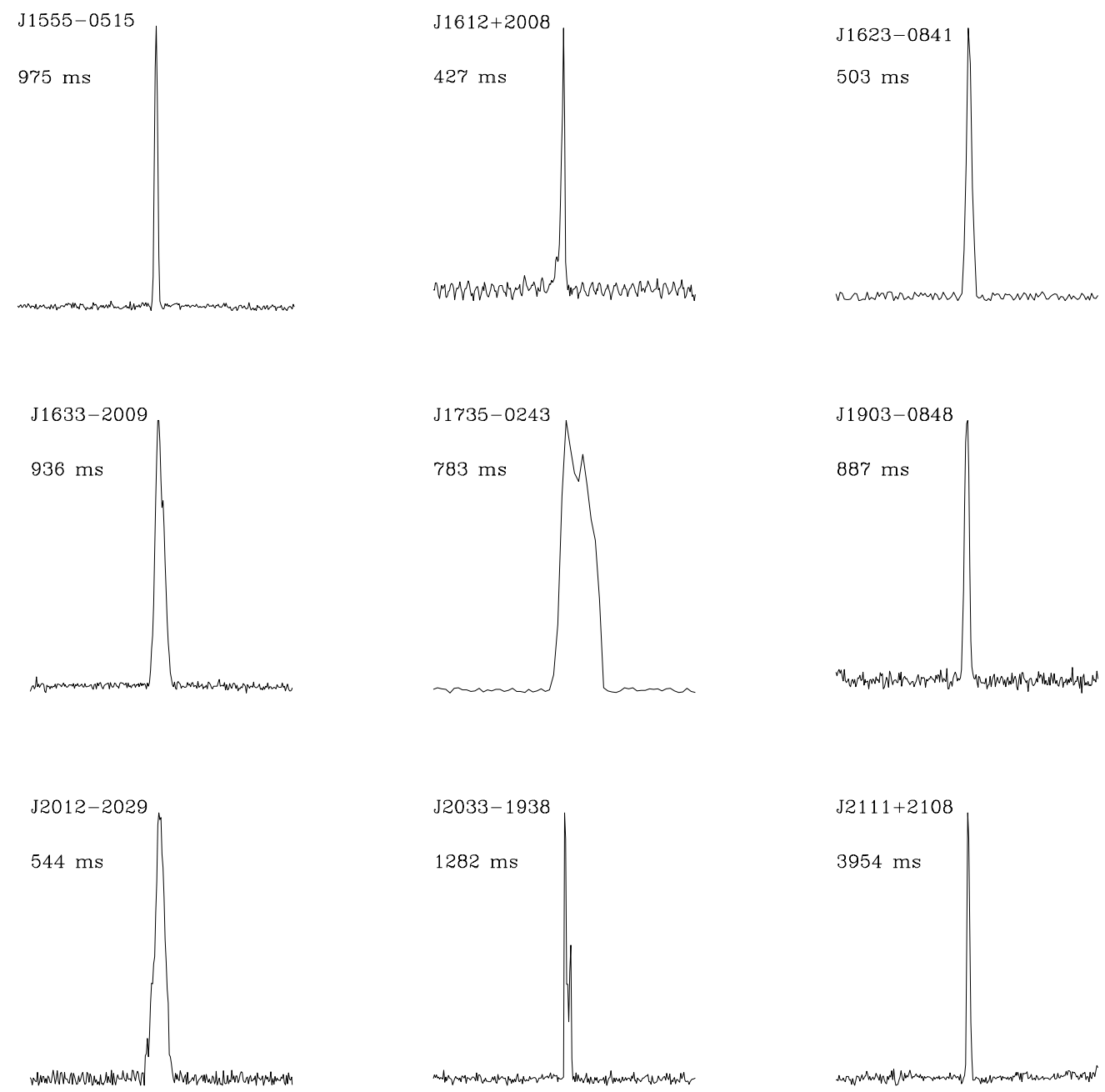

Figure 2.8: Profiles at $820 \mathrm{MHz}$ for nine newly discovered pulsars created from multi-epoch data. 

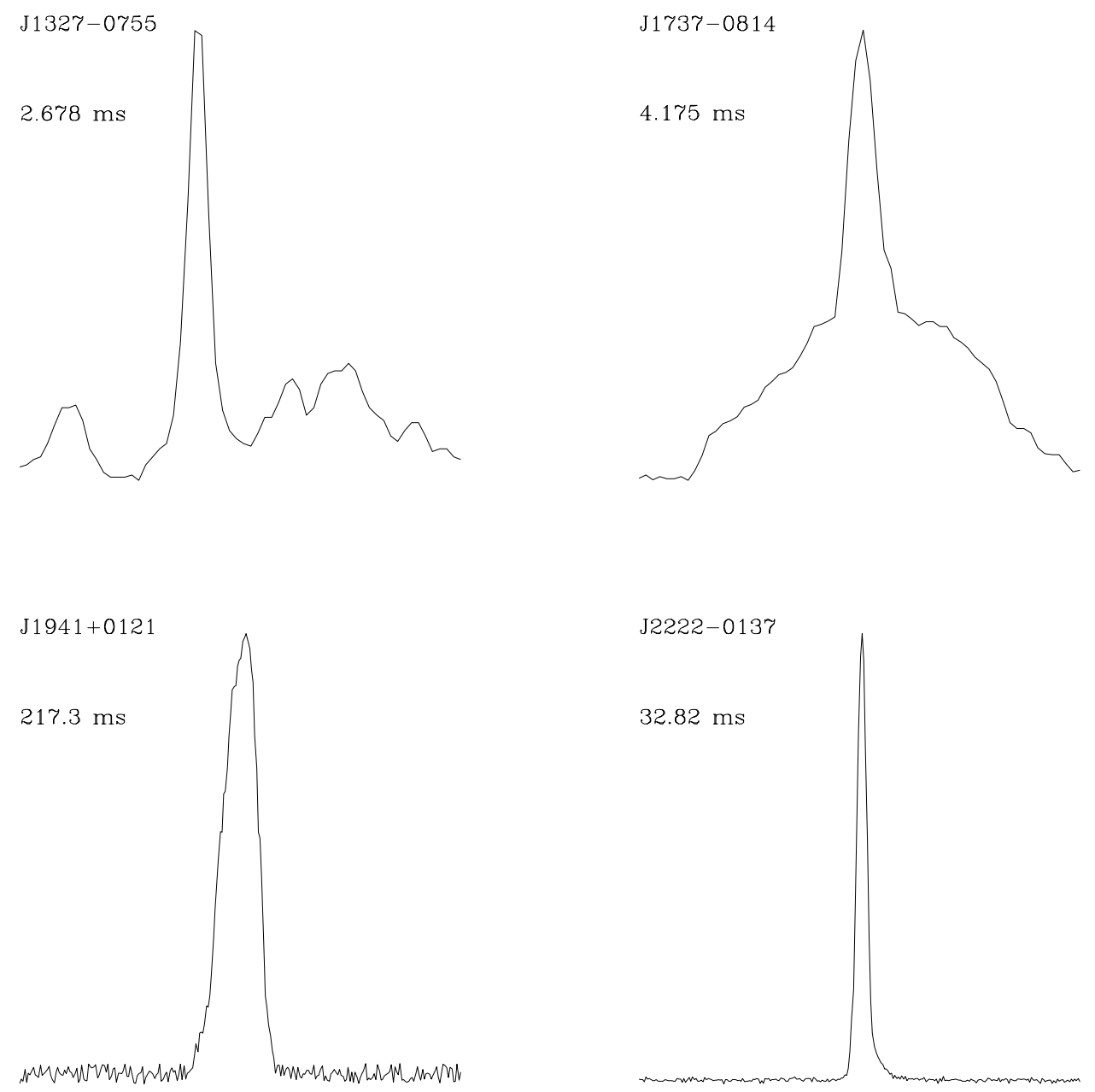

Figure 2.9: Profiles at $820 \mathrm{MHz}$ for four newly discovered pulsars created from multi-epoch data. 


\section{Chapter 3}

\section{Timing Observation of RRATs}

\subsection{Introduction}

Rotating radio transients (RRATs) are neutron stars that emit radio pulses that are not detectable using frequency domain techniques but are seen in time domain analysis. The first RRATs were discovered in a re-processing of the PMPS using a single-pulse search (see Section 1.4.3 for a description of this process). There were 11 of these presented in McLaughlin et al. (2006). Since then about 56 RRATs in total have been discovered ${ }^{1}$ and the single pulse search (described in section 1.4.3) used to detect them has become a part of all standard search pipelines.

When RRATs were initially discovered, their exact nature was not known. As time went on and more observations of them occurred, it became apparent that they were neutron stars with emission properties that deviated from those of normal pulsars because the RRATs could not be detected in a periodicity search like most known pulsars had been. Many theories have been put forward to explain the RRATs including radio emission being disrupted by fallback of supernova material (Li 2006), trapped plasma being released from radiation belts (Luo \& Melrose 2007), and circumstellar material interacting with the pulsar (Cordes \& Shannon 2008). RRATs may be just one part of the neutron star spectrum, and an analogy can be

\footnotetext{
${ }^{1}$ http://www.as.wvu.edu/ pulsar/rratalog/
} 
proposed by looking at observations of PSR B0656+14. If this pulsar were located at 12 times its current distance, it too would only be found in a single-pulse search (Weltevrede et al. 2006).

In the following sections we will discuss some of the properties that differ between pulsars and RRATs. In Section 3.2 the discussion will include the different techniques that are used to time the RRATs. In Section 3.3, we will discuss the timing results and properties of individual RRATs. In Section 3.4 we will present further work that will be done with these data to explore the RRATs population.

\subsection{Timing and Analysis Techniques}

\subsubsection{TOAs}

The RRATs' sporadic emission makes them difficult to time using standard pulsar timing techniques. In the standard procedure, the data is folded for the entire length of the observation to measure a pulse time-of-arrival (TOA), but with RRATs no pulse profile is detected when this is done. In spite of this fact, TOAs can still be created for a observation; we will describe two ways of doing this.

The first method is the simplest and involves using just a section of data where many bright pulses are seen. The data can be split up in time and only parts of the data where the pulsar is 'on' will be used to fold the data and measure a TOA. This has no disadvantages compared to the normal process and is also used when strong RFI affects parts of the observations of regular pulsars.

The second method involves using just a single pulse to measure a TOA. In 
this method the data are not folded, but are just binned for a single pulse period. This requires knowledge of when a single pulse occurs in a time series. A few disadvantages exist using this method. Usually (almost always) pulsar's single pulse will not be as bright as a folded pulse profile would. This is not true for RRATs. The error on a TOA can be expressed by the pulse width $(W)$ and $\mathrm{S} / \mathrm{N}$ by

$$
\sigma_{\mathrm{TOA}} \simeq \frac{W}{\mathrm{~S} / \mathrm{N}} \propto \frac{S_{\mathrm{sys}}}{\sqrt{t_{\text {obs }} \Delta f}} \times \frac{P \epsilon^{3 / 2}}{S_{\mathrm{mean}}}
$$

where $S_{\text {sys }}$ is the system equivalent flux density, $t_{o b s}$ is the observation time, or the pulse period for a single pulse, $\Delta f$ is the observed bandwidth, $\epsilon$ is the duty cycle $(W / P)$, and $S_{\text {mean }}$ is the mean flux density of the source. For a given pulsar or RRAT an increase in observation time will decrease the error of a TOA. In addition the emission of individual pulses does not occur at exactly the same pulse phase, a phenomenon called pulse jittering. This is seen in regular pulsars and can be seen in TOAs of RRATs that are created from a single observation (see Figure 3.1). Both of these phenomena decrease our ability to accurately time RRATs but do not prevent us from timing them.

Aside from the slicing up of the data, TOAs for RRATs are produced by the same algorithm as regular pulsars though it is not necessary. In McLaughlin et al (2006) the peak of the individual pulses in the time domain were used instead of transferring it the the Fourier domain to find the fiducial point for the TOA. 


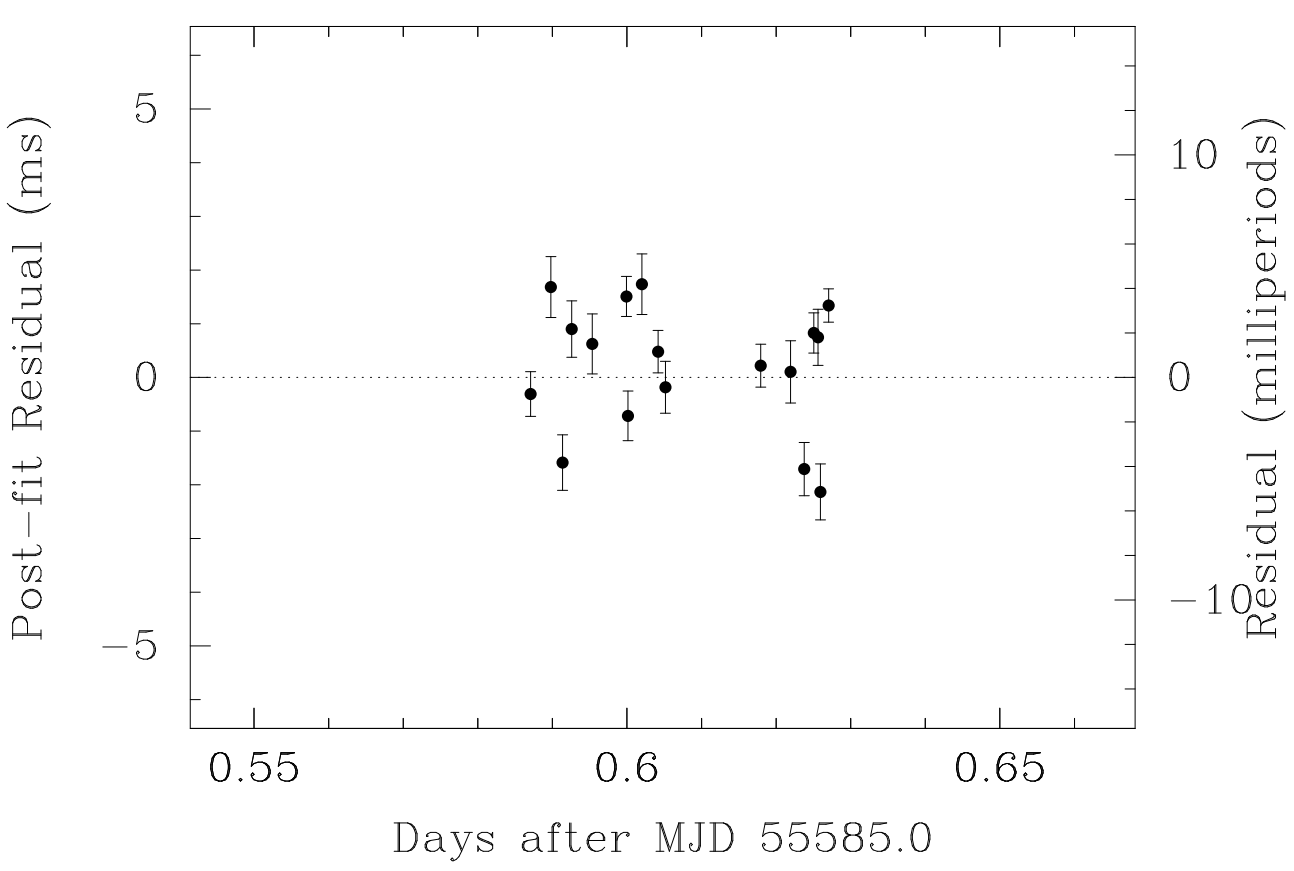

Figure 3.1: A single observation plot for PSR J1848-12 showing TOAs varying by more than the error bars on short timescales, possibly indicating that pulse jittering is occurring.

\subsubsection{Pulse Profiles and Widths}

Pulse widths $\left(W_{50}\right)$ are measured from summed pulse profiles which are created through aligning the observations using polycos (a set of polynomial coefficients that predicts when the pulse will arrive) and summing the observations together. Except for PSR J1739-2521 and PSR J1839-0141, all observations where the RRAT was detected were summed together. For PSR J1739-2521 and PSR J1839-0141, the data were used only when the pulsar was on. $W_{50}$ values were measured by fitting a single Gaussian to the summed profile with the exception of PSR J1739-2521, where the $W_{50}$ value was taken across both peaks at the FWHM of each peak. 


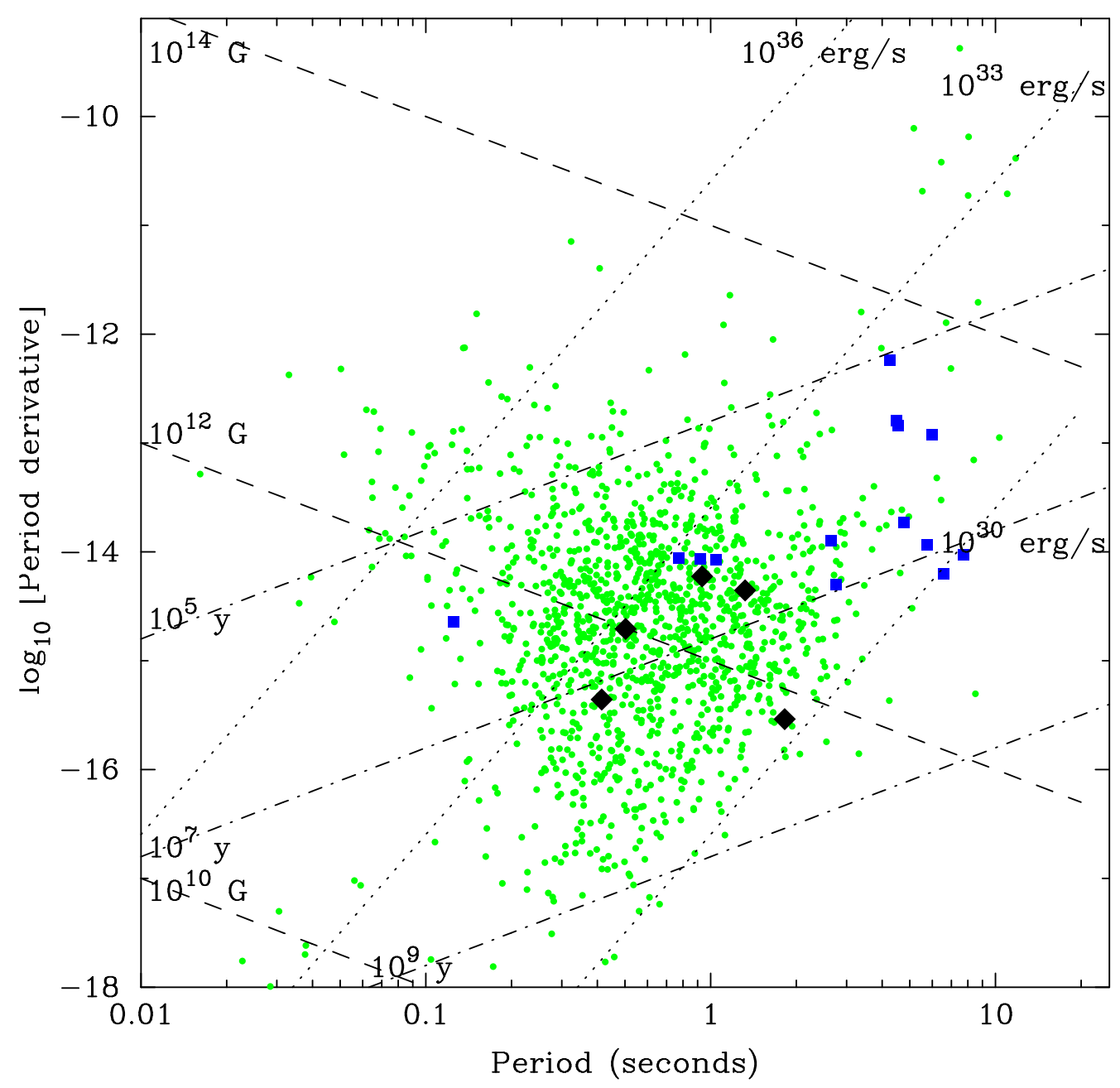

Figure 3.2: A $P-\dot{P}$ diagram showing the new RRATs as black diamonds and previously timed RRATs as blue squares. The pulsar population is taken from the ATNF pulsar catalog (Manchester et al. 2005). 


\subsection{Timing Results}

The timing-derived parameters for all the RRATs presented in this work are given in Tables 3.3-3.5. Figure 3.2 show a $P-\dot{P}$ diagram containing the new RRATs. In the following subsections some properties of each RRAT will be discussed. All the RRATs with the exception of PSR J1623-0841 were discovered in the PMPS.

\subsubsection{PSR J1623-0841}

PSR J1623-0841 was discovered only in the single-pulse search during processing of the GBT 350-MHz drift-scan pulsar survey and was labeled a RRAT. Later observations at $350 \mathrm{MHz}$ with Spigot showed single pulses but no detections were made at $820 \mathrm{MHz}$. Gridding observations were unable to localize the position better then the discovery position because of PSR J1623-0841's transient nature. The final TEMPO position was $20^{\prime}$ away from the discovery position (outside the GBT's $820-\mathrm{MHz}$ beam) and a timing solution was only attainable with the increased sensitivity of GUPPI and a dense set of observations to obtain a phase coherent timing solution at $350 \mathrm{MHz}$.

With a huge difference between observation pointing, a comparison can be made between each observation. An offset in position during an observation acts like a decrease in gain of the telescope, thus decreasing the sensitivity. Figure 3.3 shows single pulses detected from PSR J1623-0841 while pointing at two different sky positions. The top plot shows an observation with a position offset of $20^{\prime}$ from the bottom plot's position which was observed much closer to the final position 
Single pulse results for 'single_J1625-08_55078_0002'

Source: 1625-08 RA (J2000): 16:25:01.9920

Telescope: GBT DEC (J2000): $-08: 46: 19.5600$

N samples: 4872192

Instrument: GUPP| $M J D_{\text {topo }}: 55078.979189814818$ Freq $_{\mathrm{ctr}}: 350.0 \mathrm{MHz}$
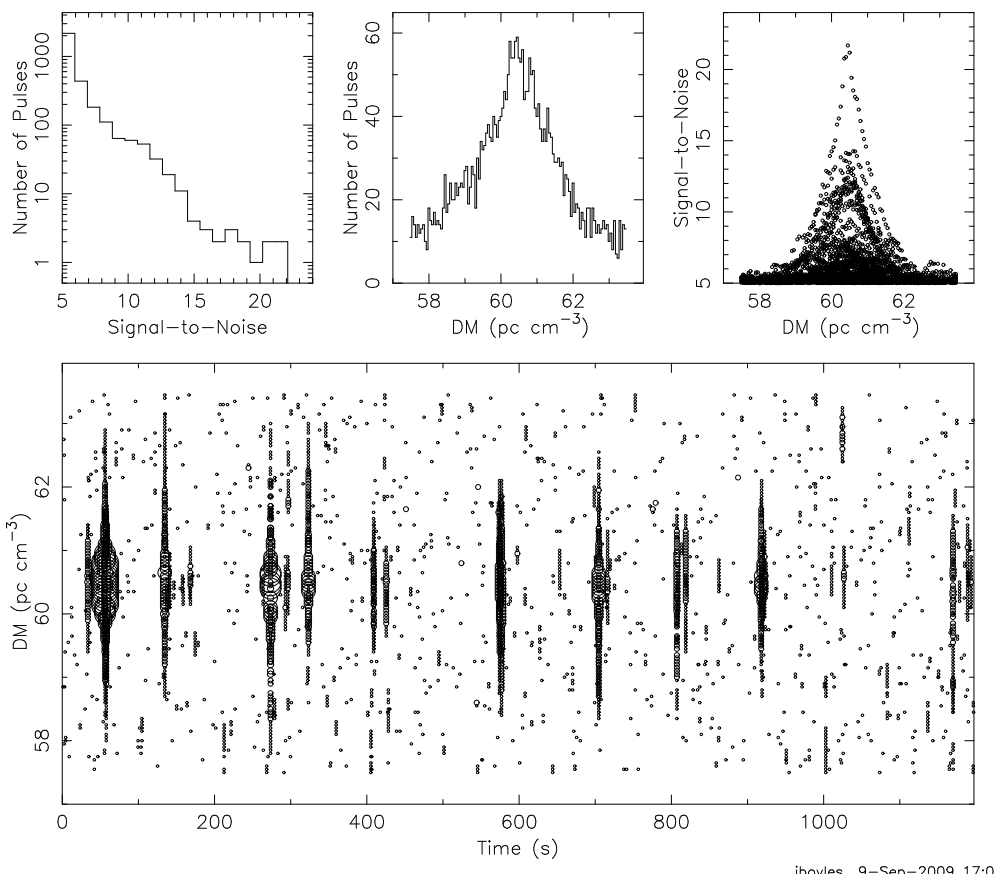

Single pulse results for 'single_J1625-08_55223_0012'

Source: $1625-08$

Telescope: GBT

Instrument: GUPPI

RA (J2000): $16: 23: 45.9360$

N samples: 3547136 DEC (J2000): $-08: 41: 29.4000 \quad$ Sampling time: $245.76 \mu \mathrm{s}$
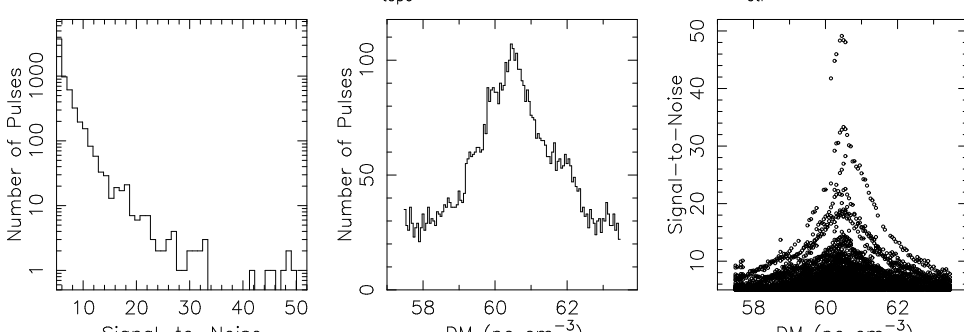

$\mathrm{DM}(\mathrm{PC}$

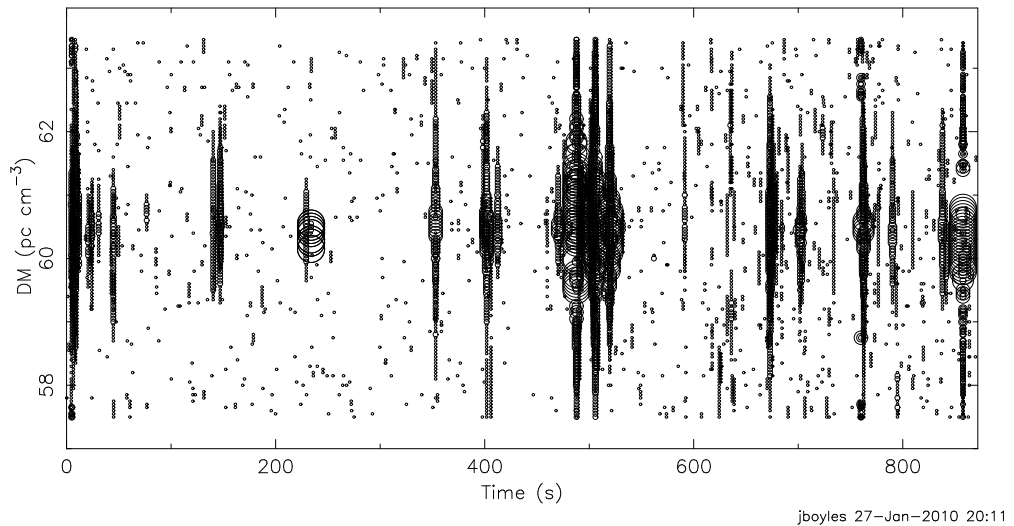

Figure 3.3: Two single-pulse detections for PSR J1623-0841 at two different pointings taken at $350 \mathrm{MHz}$ with the GBT. The top is offset by $20^{\prime}$ and the bottom is on source. 

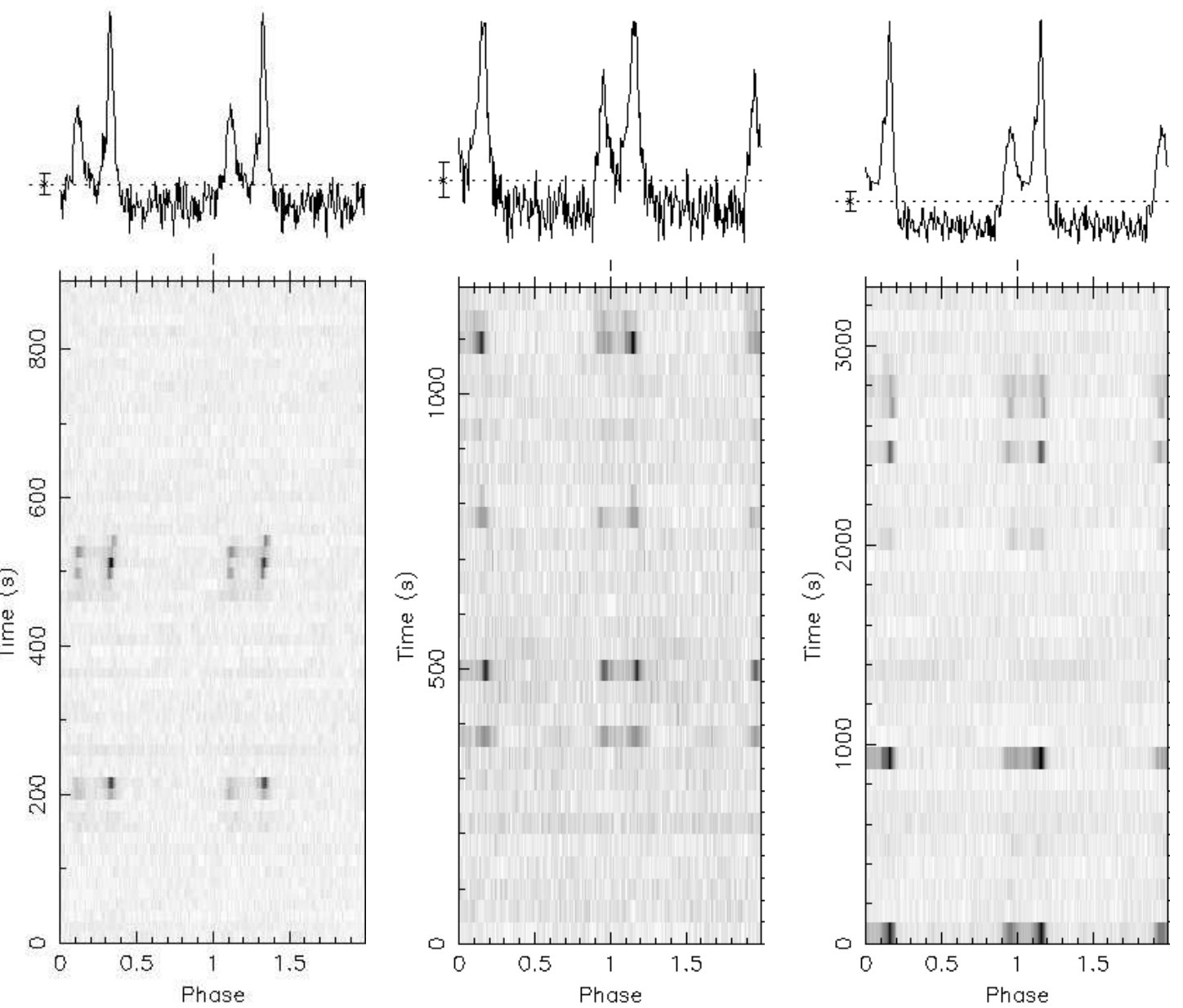

Figure 3.4: Three separate observations of PSR J1739-2521 showing the intermittent nature of this source.

of PSR J1623-0841. The bottom plot shows a higher pulse rate and pulses with higher signal to noise indicating our observations may be sensitivity limited, i.e. we are not seeing all the pulses because we do not have enough sensitivity. However a difference in system temperature between each observation could cause the same result and can not be ruled out. 


\subsubsection{PSR J1739-2521}

PSR J1739-2521 is the longest period RRAT presented in this work and is the only one that does not have a simple single Gaussian profile. The pulse profile can be seen in Figure 3.4. PSR J1739-2521 has a large offset in position between the initial observations and final position but unlike PSR J1623-0841 does not seem to have a higher pulse detection rate, which could be an indication that the radio emission is truly turning on and off.

When PSR J1739-2521 was discovered, it was inferred to have a period of $\sim 1.2$ seconds. Further analysis showed this was not correct. The reason for this incorrect period was due to the fact that both peaks produce single pulses that are detected in the single-pulse search. This causes the algorithm that determines the period from single pulses to fail. Both peaks produce detections in the search, making it hard to determine a pulse rate for the source. Each detection must be looked at by eye to determine if a single pulse actually produced two detections in the single-pulse search.

\subsubsection{PSR J1754-3014}

PSR J1754-3014 was originally reported in McLaughlin et al. (2006) with a period of 0.442617 seconds and a DM of $98 \mathrm{pc} \mathrm{cm}^{-3}$. In Keane et al (2011) it was reported to have a period of 1.32049 seconds and a DM of $293 \mathrm{pc} \mathrm{cm}^{-3}$. Here we report a period of 1.32049 seconds and a DM of $99.4 \mathrm{pc} \mathrm{cm}^{-3}$. The difference between our period and McLaughlin et al. (2006) is due to the misidentification of 
a terrestrial radio pulse as astrophysical pulse (Keane et al. 2011). The difference between our DM and Keane et al (2011) is due to PSR J1754-3014's DM being mixed up with PSR J1839-0141's new DM. McLaughlin et al. (2006) reported that there was a $32 \%$ chance that this RRAT had a different period due to low pulse counts.

\subsubsection{PSR J1839-0141}

PSR J1839-0141 was originally reported in McLaughlin et al. (2006) to have a period of 0.93190 seconds and a DM of $307 \mathrm{pc} \mathrm{cm}^{-3}$. Here it is reported with a period of 0.93326 seconds and a DM of $293.4 \mathrm{pc} \mathrm{cm}^{-3}$. The difference in DM may be due to the coarse frequency resolution of the PMPS and is less than a $2 \sigma$ difference from the discovery DM. The discovery period is different by $1.5 \mathrm{~ms}$ but it was reported in McLaughlin et al. (2006) that there was a small probability that the period may be different due to low pulse counts and low detection rate. The difference between the discovery period and the period reported here is much larger

than what would be produced by the measured $\dot{P}$. It was only seen in one of ten observations reported in McLaughlin et al. (2006) and has never been seen again with the Parkes telescope since its discovery.

Table 3.1 contains all the observations of PSR J1839-0141 used in this work. The longest single observation of PSR J1839-0141 without a detection is 3593 seconds. If this is used calculate a flux density limit for when PSR J1839-0141 is not emitting, a limit of $5.9 \mu \mathrm{Jy}$ is obtained assuming a detection limit of $6 \sigma$ for 


\begin{tabular}{|c|c|c|c|c|}
\hline $\begin{array}{l}\text { topocentric MJD } \\
\text { start time } \\
\text { (days) }\end{array}$ & $\begin{array}{c}\text { time } \\
\text { visible } \\
\text { (seconds) }\end{array}$ & $\begin{array}{c}\text { start time } \\
\text { since start MJD } \\
(\text { seconds })\end{array}$ & $\begin{array}{c}\text { stop time } \\
\text { since start MJD } \\
\text { (seconds) }\end{array}$ & $\begin{array}{l}\text { observation } \\
\text { length } \\
\text { (seconds) }\end{array}$ \\
\hline 55011.205081018517 & 104 & 1892 & 1996 & 3901 \\
\hline 55013.207037037035 & 90 & 1785 & 1875 & 2697 \\
\hline 55041.079328703701 & 65 & 123 & 188 & 2996 \\
\hline 55186.809212962966 & 120 & 720 & 840 & 1197 \\
\hline 55267 & & & & 3593 \\
\hline 55303.439293981479 & 98 & 1800 & 1898 & 6147 \\
\hline 55339.340335648150 & 128 & 504 & 642 & 6588 \\
\hline 55339.340335648150 & 60 & 1202 & 1262 & \\
\hline 55339.340335648150 & 58 & 2937 & 2995 & \\
\hline 55359 & & & & 3394 \\
\hline 55401.184699074074 & 56 & 1240 & 1296 & 5575 \\
\hline 55432 & & & & 3593 \\
\hline 55460 & & & & 5172 \\
\hline 55494 & & & & 3593 \\
\hline 55522 & & & & 3593 \\
\hline 55550.663599537038 & 307 & 1757 & 2064 & 3593 \\
\hline 55585.547928240740 & 97 & 873 & 970 & 3593 \\
\hline 55612.549386574072 & 50 & 1742 & 1792 & 2838 \\
\hline 55613.544618055559 & 33 & 2185 & 2218 & 4193 \\
\hline 55617.601006944446 & 95 & 687 & 782 & 1008 \\
\hline 55660 & & & & 3593 \\
\hline 55707 & & & & 3593 \\
\hline 55737.329652777778 & 55 & 2344 & 2399 & 3593 \\
\hline 55780.077858796300 & 14 & 676 & 690 & 3593 \\
\hline 55825.914444444446 & 55 & 0 & 55 & 3593 \\
\hline
\end{tabular}

Table 3.1: Table containing all observations of PSR 1839-0141. Integer MJDs have no detection of PSR 1839-0141 in either periodicity searches or single-pulse searches. 
emission at $820 \mathrm{MHz}$. If the assumption that PSR J1839-0141 is not truly emitting while off, then a null fraction of $98.3 \%$ is calculated by taking the total time on and dividing it by the total observed time.

\subsubsection{PSR J1848-1243}

PSR J1848-1243 was originally reported with a period of 6.7953 seconds and here we report it to have a period of 0.41438 seconds which is a factor of 16.40 different. PSR J1848-1243 had a 32\% chance that its period was incorrect when it was discovered due to low pulse counts (McLaughlin et al. 2006).

Figure 3.5 shows both a single-pulse detection and folded detection of PSR J1848-1243. The pulsar can be seen in clearly in both detections though weakly in the folded detection. Also note that PSR J1848-1243 can be seen throughout the whole observation time in the folded detection but only about 20 pulses are seen in the single-pulse plot. This is the trend of many pulsars and PSR J1848-1243 may not be a RRAT, just a pulsar missed in the periodicity search during the many processing of the PMPS.

\subsection{Summary and Further Work}

We present pulsar timing solutions for five of the nineteen that have been obtained for the RRATs population (McLaughlin et al. 2006, McLaughlin et al. 2009, Keane et al. 2011). Obtaining timing solutions is just the first step in understanding this unique population. Further analysis to be conducted includes looking for long 
Single pulse results for 'single_J1848-12_55825_0010'

Telescope: GBT

Instrument: GUPPI
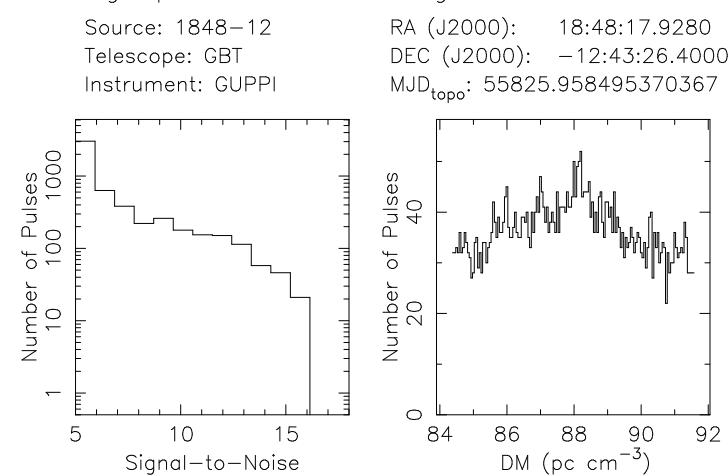

N samples: 7073792

Sampling time: $24576 \mu \mathrm{s}$

Freq $_{\mathrm{ctr}}: 820.0 \mathrm{MHz}$
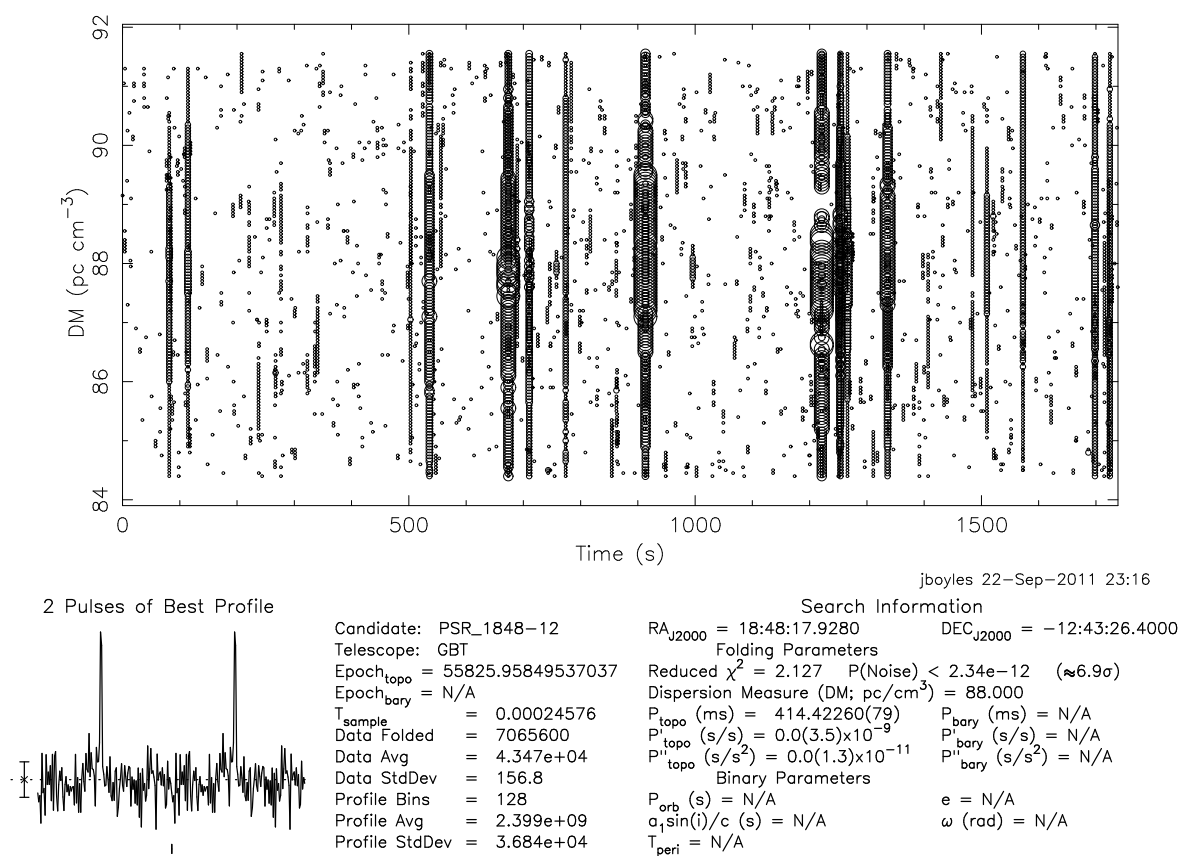

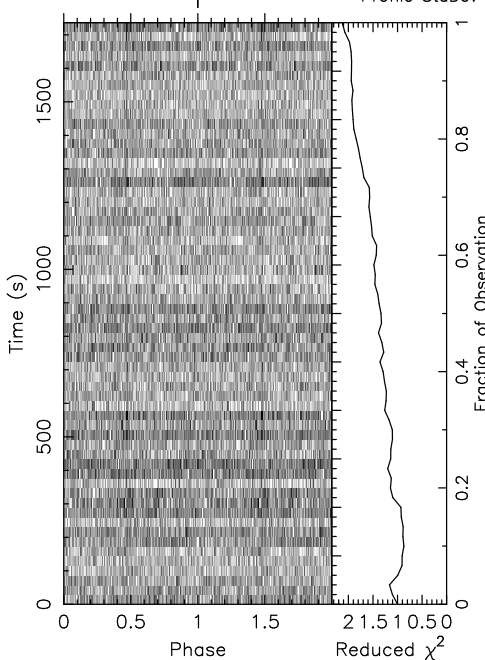

guppi_J1848-12_55825_0010_DM88.00.sub00

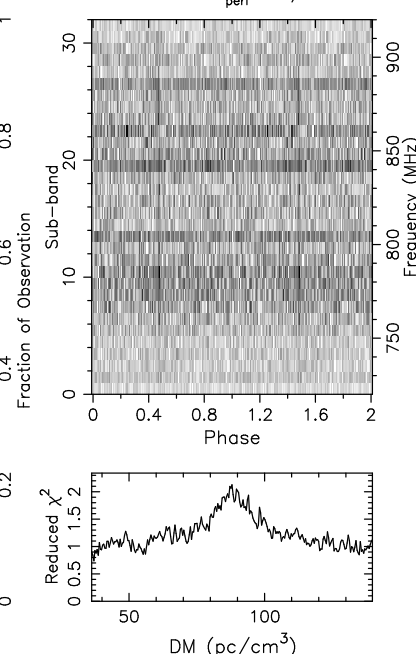

$\mathrm{DM}\left(\mathrm{pc} / \mathrm{cm}^{3}\right)$

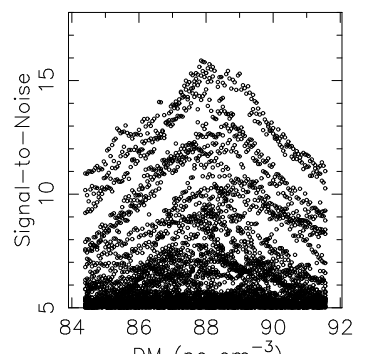

$\begin{array}{lcc}86 & 88 & 90 \\ D M & \left(p c \mathrm{~cm}^{-3}\right)\end{array}$

\section{.}




\begin{tabular}{lccccc}
\hline $\begin{array}{l}\text { RRAT } \\
\text { PSRJ name }\end{array}$ & $\begin{array}{c}\text { Discovery RaJ } \\
\text { HH:MM:SS }\end{array}$ & $\begin{array}{c}\text { Discovery DecJ } \\
\text { DD:MM:SS }\end{array}$ & $\begin{array}{c}\text { Final RaJ } \\
\text { HH:MM:SS }\end{array}$ & $\begin{array}{c}\text { Final DecJ } \\
\text { DD:MM:SS }\end{array}$ & $\begin{array}{c}\Delta \text { Position } \\
\text { arcmin }\end{array}$ \\
\hline J1623-0841 & $16: 25: 02$ & $-08: 46: 20$ & $16: 23: 42$ & $-08: 41: 36$ & 20.55 \\
J1739-2521 & $17: 39: 50$ & $-25: 13: 16$ & $17: 39: 33$ & $-25: 21: 16$ & 9.06 \\
J1754-3014 & $17: 54: 16$ & $-30: 11: 37$ & $17: 54: 30$ & $-30: 14: 43$ & 4.67 \\
J1839-0141 & $18: 39: 53$ & $-01: 36: 21$ & $18: 39: 07$ & $-01: 41: 56$ & 12.8 \\
J1848-1243 & $18: 48: 02$ & $-12: 47: 23$ & $18: 48: 17$ & $-12: 43: 26$ & 5.45 \\
\hline
\end{tabular}

Table 3.2: Table containing the discovery position and final position for five RRATs. PSR J1739-2521 and PSR J1839-0141 were both discovered outside the FWHM of the telescope beam size of their survey. PSR J1623-0841 was discovered at 350 $\mathrm{MHz}$ with the GBT and its beam is $\sim 36^{\prime}$.

term periodicities in the detection rates of PSR J1839-0141 and PSR J1739-2521. Both seem to turn on and off and may have an underlying periodicity associated with them. PSR J1839-0141 is the most promising because it is only seen at most once per observation while PSR J1739-2521 shows up multiple times with different cadences within an individual observation.

Statistical comparisons between the pulsar and RRAT populations may provide additional insight to the RRATs population. From a simple look at Figure 3.2 it seems like the RRATs have longer periods, higher magnetic fields, and smaller characteristic ages than the population of normal pulsars as a whole. Further work with the Kolmogorov-Smirnov test (KS-test), following that which was done in Keane et al (2011), will quantify these results and provide supporting evidence for such statements.

The five RRATs reported in this work are detectable without the single-pulse search and may be more like normal pulsars than previously thought. The RRATs may actually be a distinct class of neutron stars but not all objects found only in 


\begin{tabular}{|c|c|c|}
\hline Parameter & PSR J1623-0841 & PSR J1739-2521 \\
\hline \multicolumn{3}{|l|}{ Timing Parameters } \\
\hline Right Ascension (J2000) & $16: 23: 42.711(10)$ & $17: 39: 32.83(10)$ \\
\hline Declination (J2000) & $-08: 41: 36.4(5)$ & $-25: 21: 16(24)$ \\
\hline Spin Period (s) & $0.503014992514(6)$ & $1.81846116407(19)$ \\
\hline Period Derivative $\left(\mathrm{s} \mathrm{s}^{-1}\right)$ & $1.9582(6) \times 10^{-15}$ & $2.9(3) \times 10^{-16}$ \\
\hline Dispersion Measure $\left(\mathrm{pc} \mathrm{cm}^{-3}\right)$ & $60.433(16)$ & 186.4 \\
\hline Reference Epoch (MJD) & 55048 & 55612 \\
\hline Span of Timing Data & $54635-55522$ & $55339-55825$ \\
\hline Number of TOAs & 36 & 32 \\
\hline RMS Residual ( $\mu \mathrm{s})$ & 764 & 4845 \\
\hline \multicolumn{3}{|l|}{ Derived Parameters } \\
\hline Galactic Longitude (degrees) & 5.77 & 2.3 \\
\hline Galactic Latitude (degrees) & 27.3 & 3.0 \\
\hline Distance $(\mathrm{kpc})$ & 3.3 & 3.5 \\
\hline Distance Off Plane (kpc) & 1.5 & 0.18 \\
\hline Surface Magnetic Field (10 12 Gauss) & 1.00 & 0.735 \\
\hline Spin-down Luminosity $\left(10^{31} \operatorname{ergs~s}^{-1}\right)$ & 19.3 & 0.00607 \\
\hline Characteristic Age (Myr) & 4.07 & 99.2 \\
\hline $\mathrm{W}_{50} 350 \mathrm{MHz}(\mathrm{ms})$ & $6.05(18)$ & $\mathrm{N} / \mathrm{A}$ \\
\hline $\mathrm{W}_{50} 820 \mathrm{MHz}(\mathrm{ms})$ & $4.81(8)$ & $497(20)$ \\
\hline
\end{tabular}

Table 3.3: Fitted and derived parameters for RRATs.

the single-pulse search are RRATs. Sensitivity limits of the current instruments or distant objects like those suggested by Weltevrede et al. (2006) make up part of this class of pulsars that are not very erratic. 


\begin{tabular}{|c|c|c|}
\hline Parameter & PSR J1754-3014 & PSR J1839-0141 \\
\hline \multicolumn{3}{|l|}{ Timing Parameters } \\
\hline Right Ascension (J2000) & $17: 54: 30.08(5)$ & 18:39:07.03(3) \\
\hline Declination (J2000) & $-30: 14: 42(6)$ & $-01: 41: 56.0(9)$ \\
\hline Spin Period $(\mathrm{s})$ & $1.3204902915(3)$ & $0.93326564072(6)$ \\
\hline Period Derivative $\left(\mathrm{s} \mathrm{s}^{-1}\right)$ & $4.424(12) \times 10^{-15}$ & $5.943(3) \times 10^{-15}$ \\
\hline Dispersion Measure $\left(\mathrm{pc} \mathrm{cm}^{-3}\right)$ & $99.38(10)$ & 293.4 \\
\hline Reference Epoch (MJD) & 55025 & 55612 \\
\hline Span of Timing Data & $54998-55585$ & $55011-55825$ \\
\hline Number of TOAs & 24 & 16 \\
\hline RMS Residual ( $\mu \mathrm{s})$ & 3612 & 1803 \\
\hline \multicolumn{3}{|l|}{ Derived Parameters } \\
\hline Galactic Longitude (degrees) & 359.8 & 30.0 \\
\hline Galactic Latitude (degrees) & -2.3 & 2.0 \\
\hline Distance (kpc) & 2.2 & 6.2 \\
\hline Distance Off Plane (kpc) & 0.08 & 0.22 \\
\hline Surface Magnetic Field (10 12 Gauss) & 2.44 & 2.38 \\
\hline Spin-down Luminosity $\left(10^{31} \mathrm{ergs} \mathrm{s}^{-1}\right)$ & 2.41 & 9.19 \\
\hline Characteristic Age (Myr) & 4.72 & 2.49 \\
\hline $\mathrm{W}_{50} 350 \mathrm{MHz}(\mathrm{ms})$ & $27.5(2)$ & $\mathrm{N} / \mathrm{A}$ \\
\hline $\mathrm{W}_{50} 820 \mathrm{MHz}(\mathrm{ms})$ & $24.6(7)$ & $7.3(2)$ \\
\hline
\end{tabular}

Table 3.4: Fitted and derived parameters for RRATs. 


\begin{tabular}{|c|c|}
\hline Parameter & PSR J1848-1243 \\
\hline \multicolumn{2}{|l|}{ Timing Parameters } \\
\hline Right Ascension (J2000) & $18: 48: 17.980(8)$ \\
\hline Declination (J2000) & $-12: 43: 26.6(5)$ \\
\hline Spin Period $(\mathrm{s})$ & $0.41438334869(2)$ \\
\hline Period Derivative $\left(\mathrm{s} \mathrm{s}^{-1}\right)$ & $4.403(17) \times 10^{-16}$ \\
\hline Dispersion Measure $\left(\mathrm{pc} \mathrm{cm}^{-3}\right)$ & 88.0 \\
\hline Reference Epoch (MJD) & 55613 \\
\hline Span of Timing Data & $55267-55825$ \\
\hline Number of TOAs & 156 \\
\hline RMS Residual ( $\mu \mathrm{s})$ & 1361 \\
\hline \multicolumn{2}{|l|}{ Derived Parameters } \\
\hline Galactic Longitude (degrees) & 21.2 \\
\hline Galactic Latitude (degrees) & -5.1 \\
\hline Distance $(\mathrm{kpc})$ & 2.4 \\
\hline Distance Off Plane (kpc) & 0.21 \\
\hline Surface Magnetic Field (10 12 Gauss) & 0.432 \\
\hline Spin-down Luminosity $\left(10^{31} \mathrm{ergs} \mathrm{s}^{-1}\right)$ & 7.78 \\
\hline Characteristic Age (Myr) & 14.9 \\
\hline $\mathrm{W}_{50} 820 \mathrm{MHz}(\mathrm{ms})$ & $4.8(5)$ \\
\hline
\end{tabular}

Table 3.5: Fitted and derived parameters for RRATs. 

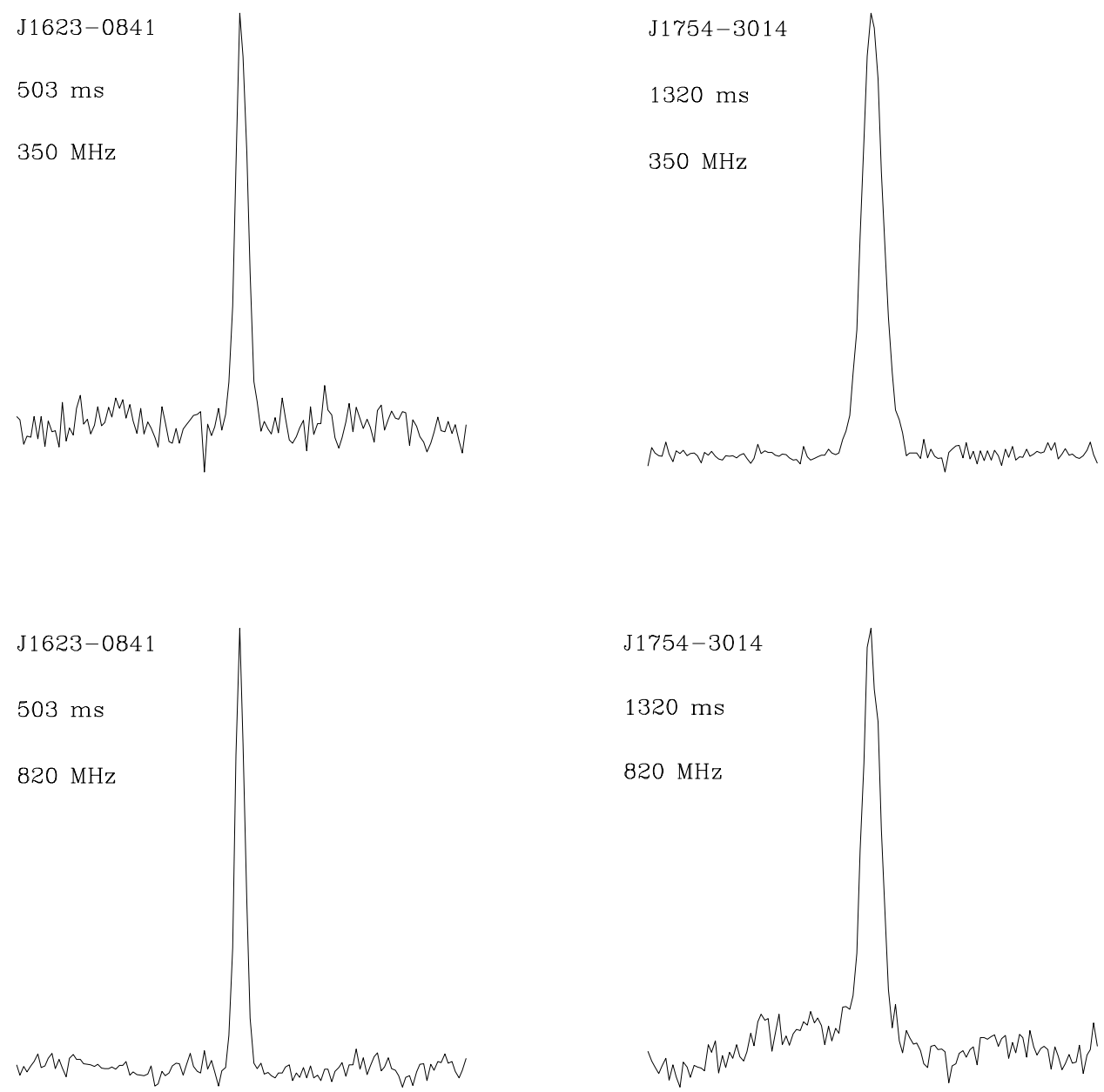

Figure 3.6: Pulse profiles for PSR J1625-0841 and PSR J1754-3014 at $350 \mathrm{MHz}$ and $820 \mathrm{MHz}$. The profiles are created by folding each observation and summing all profiles together. 

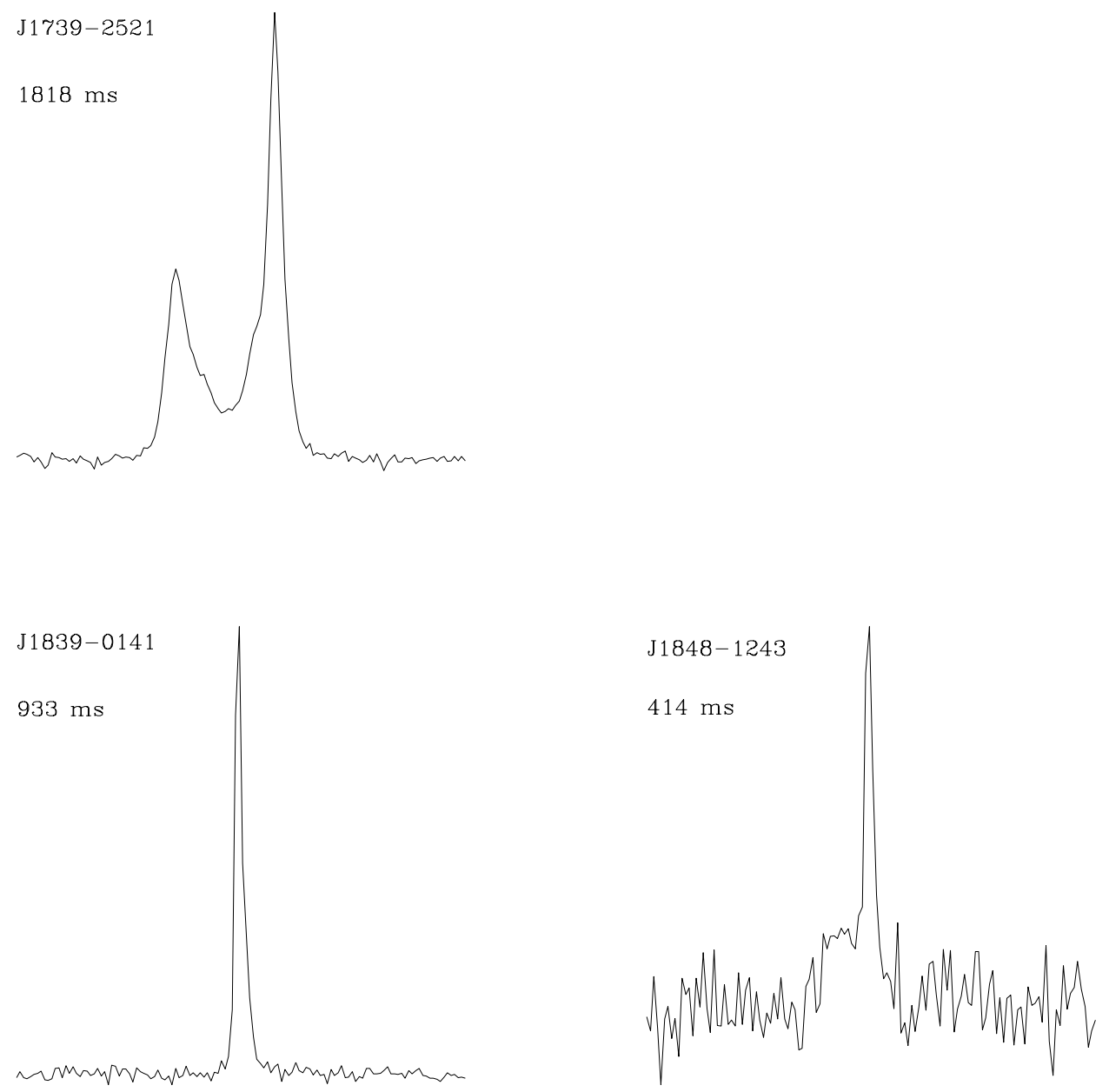

Figure 3.7: Pulse profile at $820 \mathrm{MHz}$ for the rest for the rest of the RRATs. Both of PSR J1739-2521 and PSR J1839-0141 profiles are a sum of data only when the pulsar is on. PSR J1848-1243's profile is created in the same way as for PSR J1625-0841 and PSR J1754-3014. 


\section{Chapter 4}

\section{Young Radio Pulsars in Galactic Globular Clusters}

Published as: Boyles, J., Lorimer, D. R., Turk, P. J., Mnatsakanov, R., Lynch, R.

S., Ransom, S. M., Freire, P. C., Belczynski, K. 2011, ApJ, 742, 51

Young Radio Pulsars in Galactic Globular Clusters

\subsection{Introduction}

Since the first discovery of PSR B1821-24 in M28 (Lyne et al. 1987), 143 pulsars $^{1}$ have been discovered in 27 globular clusters (GCs). While the majority of GC pulsars are thought to have been formed in low-mass X-ray binary systems, in which the neutron star has been spun up to millisecond periods by the transfer of matter from an evolved companion (see, e.g. Camilo \& Rasio 2005 for a recent review), a small minority of the GC pulsar population appears to be similar to the isolated "normal" pulsars which inhabit the disk of our Galaxy (i.e. spin periods $(P)$ of several hundred ms, characteristic ages $\left(\tau_{c}\right)$ of $10^{7}-10^{8} \mathrm{yr}$, and inferred dipole magnetic field strengths $(B)$ of $10^{11}-10^{12}$ Gauss). These pulsars were originally discussed by Lyne et al. (1996) who noted that, since no significant star formation has occurred in GCs in the last billion years (Briley et al. 1994), this population appears to be highly anomalous. Lyne et al. (1996) also note that the young pulsars

${ }^{1}$ For an up-to-date list of known globular cluster pulsars,
http://www.naic.edu/ pee


appear in metal-rich GCs. This trend has persisted despite fifteen years of intense searches of most of the cluster population. A goal of this work is to investigate the statistical and astrophysical significance of this result.

Recent improvements in observational systems over the past decade have led to a wealth of discoveries of pulsars in GCs. Sensitive radio surveys for pulsars have been conducted on almost 100 GCs to date. The two most fruitful have been Terzan 5 (Ransom et al. 2005) and 47 Tucanae (Freire et al. 2001), with 34 and 23 pulsars respectively. Neither of these clusters harbor any normal pulsars, which we will henceforth define as being pulsars with spin periods $P>100 \mathrm{~ms}$ and inferred magnetic field strengths $B>10^{11}$ Gauss. This is somewhat surprising, given that the significant selection effects known to hamper the detection of binary and millisecond pulsars in clusters (Camilo \& Rasio 2005) are not as severe for normal isolated pulsars.

Clearly, some physical mechanism is at work which produces these apparently young pulsars in GCs in a different way to how we believe they are formed in the disk of our Galaxy. One possible method is the collapse of a white dwarf via an electron capture supernova in a binary or collisional system (Ivanova et al. 2008). In this paper, we revisit the statistics of the normal pulsars based on the results of recent surveys, and recent studies of the Galactic population of normal pulsars (Faucher-Giguére \& Kaspi 2006 (hereafter FK06); Ridley \& Lorimer 2010). In §4.2, we review the current sample of normal pulsars in GCs. In $\S 4.3$, we compile a list of flux-density detection limits for 97 GCs based on published searches and some recent unpublished results. In $\S 4.4$, we use these limits to characterize the population of 
young pulsars. In $\S 4.5$ we discuss the intrinsic population and birth rate of young pulsars in GCs. In $\S 4.6$ we discuss formation scenarios and future work with young globular cluster pulsars.

\subsection{The current sample of young pulsars in GCs}

At least three, possibly four, young pulsars are known in two or three GCs. These four pulsars are PSR B1718-19 in NGC 6342, PSR B1745-20 in NGC 6640, and PSR J1823-3021B and PSR J1823-3021C in NGC 6624. The properties of these pulsars are summarized in Table 4.2 and their GC properties relevant to this data analysis can be seen in Table 4.1. As can be seen by inspecting their position with respect to other normal Galactic field pulsars on the $P-\dot{P}$ diagram shown in Figure 4.1, these pulsars appear to be consistent with the distribution of normal pulsars in the Galactic disk.

Two long-period pulsars are not included in this compilation. Firstly, the 110ms pulsar PSR B2127+11A in GC NGC 7078 which is known to have a negative period derivative due to contamination by the cluster potential and nearby stars (Wolszczan et al. 1989). As discussed by these authors, the pulsar most likely has a small intrinsic period derivative indicating that it has undergone some recycling in a binary system which has subsequently been disrupted due to close encounters in the

cluster. It is also noted that a recent measurement of the second period derivative of $\ddot{P}=3.2 \times 10^{-29} s^{-1}$ for this pulsar is entirely consistent with encounters with nearby stars in the cluster (Jacoby et al. 2006). Another long-period pulsar which we do 
not consider to be young is the 110-ms pulsar PSR J1750-37A in GC NGC 6441 (Freire et al. 2008). This is a member of an eccentric binary system and its low period derivative places it closer to the region of the $P-\dot{P}$ diagram occupied by the double neutron star binary systems and the eccentric neutron star-white dwarf binaries (see Figure 4.1).

One other caveat needs to be discussed with the sample of young pulsars present in this chapter. PSR B1718-19 may not truly be a member of NGC 6342 and is the only pulsar located near NGC 6342. An earlier discussion of this subject is presented in Bailes et al. (2005) and a further expansion will be presented here. The original paper used PSR B1718-19's position, binary status, and dispersion measure (DM) to argue for its association with NGC 6342. Its position of $2.3^{\prime}$ away from the cluster's center is three times greater then the half-mass radius of the cluster. All other young pulsars presented in Table 4.1 and a majority of all globular cluster pulsars with timing solutions are found within the half-mass radius. The DM of $75.7 \mathrm{pc} \mathrm{cm}^{-3}$ for PSR B1718-19 is somewhat below the predicted values of $120 \mathrm{pc} \mathrm{cm}^{-3}$ using $\mathrm{DM}=20 / \sin (b) \mathrm{pc} \mathrm{cm}^{-3}$ (Lyne et al. 1995) where $b=9.72^{\circ}$, $130 \mathrm{pc} \mathrm{cm} \mathrm{cm}^{-3}$ (Taylor \& Cordes 1993), or $229 \mathrm{pc} \mathrm{cm}^{-3}$ (Cordes \& Lazio 2002). These DM models are uncertain, but factors in the range of 1.5 to 3 are larger than the known differences for sources within $|b|<10^{\circ}$ of the Galactic plane. The unusual binary nature of PSR B1718-19 gives support to its association with NGC 6342 due to its existence as an eclipsing low-mass binary pulsar (Freire 2005) and due to the fact that the proportion of pulsars with binary companions is about two orders of magnitude greater for GC pulsars than all Galactic field pulsars (Lyne et 


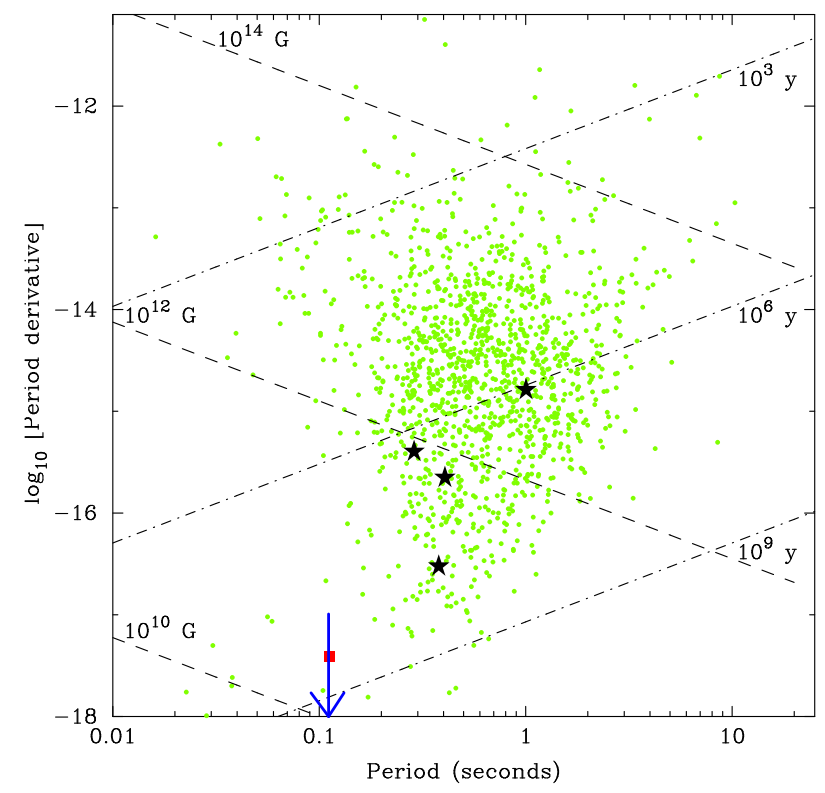

Figure 4.1: $P-\dot{P}$ diagram showing young globular cluster pulsars as black stars and PSR J1750-37A as a red square. PSR B2127+11A is shown as a blue arrow with the arrow representing the limit on period derivative at the bottom of the diagram. The pulsar population data are taken from the ATNF pulsar database (Manchester et al 2005).

al. 1995). In the following sections, given the lack of clear evidence for or against the association, the results are discussed both with and without the inclusion of PSR B1718-19 and it will be explicitly stated when PSR B1718-19 is not included.

$\begin{array}{lllll}\text { PSR } & \text { B1718-19 } & \text { B1745-20 } & \text { J1823-3021B } & \text { J1823-3021C } \\ \text { Cluster } & \text { NGC 6342 } & \text { NGC 6440 } & \text { NGC 6624 } & \text { NGC 6624 } \\ P(\mathrm{~ms}) & 1004 & 288 & 379 & 406 \\ \dot{P}\left(\mathrm{~s} \mathrm{~s}^{-1}\right) & 1.6 \times 10^{-15} & 4.0 \times 10^{-16} & 3.0 \times 10^{-17} & 2.2 \times 10^{-16} \\ \text { DM }\left(\mathrm{pc} \mathrm{cm}{ }^{-3}\right) & 75.7 & 219.4 & 86.9 & 86.7 \\ B(\mathrm{G}) & 1.3 \times 10^{12} & 3.4 \times 10^{11} & 1.1 \times 10^{11} & 3.0 \times 10^{11} \\ \tau_{c}(\mathrm{yrs}) & 9.8 \times 10^{6} & 1.1 \times 10^{7} & 2.0 \times 10^{8} & 2.9 \times 10^{7} \\ S_{1400}(\mathrm{mJy}) & 0.30 & 0.37 & 1.04 & 0.71 \\ \text { Binary? } & \text { Yes } & \text { No } & \text { No } & \text { No } \\ \text { Reference } & \text { Lyne et al. (1993) } & \text { Freire et al. (2008) } & \text { Lynch et al. }(2011) \text { Lynch et al. (2011) }\end{array}$

Table 4.1: Properties of the four young pulsars currently known in GCs. 


\subsection{Sensitivity limits for young pulsars in GCs}

To carry out the statistical analysis of this sample, it is necessary to have a substantial compilation of upper limits from GCs that have been searched for such pulsars. Table 4.3 gives our compilation of flux density limits at $1400 \mathrm{MHz}$ for searches of each cluster, $S_{\min }$, and GC properties relevant to this work. The main surveys that were used are Hessels et al. (2007), Lynch \& Ransom (2011), Lynch et al. (2011), and Possenti et al. (2010). The rest of the GCs had their flux limits taken from the most recent discovery paper. If the paper did not quote a flux limit, one was derived using the survey parameters from the paper and the radiometer equation for pulsars which gives:

$$
S_{\min }=\frac{\beta\left(\mathrm{S} / \mathrm{N}_{\text {min }}\right) \mathrm{T}_{\mathrm{sys}}}{G \sqrt{n_{\mathrm{p}} t_{\mathrm{int}} \Delta f}} \sqrt{\frac{\delta}{1-\delta}},
$$

where $\beta$ is a digitization correction faction, $\mathrm{S} / \mathrm{N}_{\min }$ is the minimum signal-to-noise ratio, $T_{\text {sys }}$ is the system temperature of the telescope, $G$ is the gain of the telescope, $n_{\mathrm{p}}$ is the number of polarizations summed, $t_{\mathrm{int}}$ is the observation time, $\Delta f$ is the bandwidth of the backend, and $\delta$ is the fractional pulse width (Lorimer \& Kramer 2005).

The parameters $t_{\text {int }}, \Delta f$, and $n_{\mathrm{p}}$ are all taken from the relevant survey paper. The values for $G$ and $\beta$ can be obtained from the websites or papers that give the telescope and backend specifications. $\mathrm{S} / \mathrm{N}_{\min }$ and $\delta$ are parameters that are chosen to have values of 8 and 0.1 respectively. $T_{\text {sys }}$ can be expressed as: 


$$
T_{\mathrm{sys}}=T_{\mathrm{rec}}+T_{\mathrm{CMB}}+T_{\mathrm{gsyn}}+T_{\mathrm{spill}}+T_{\mathrm{RFI}}
$$

where $T_{\text {rec }}$ is the receiver temperature, $T_{\mathrm{CMB}}$ is the cosmic background temperature, $T_{\text {gsyn }}$ is the Galactic synchrotron temperature, $T_{\text {spill }}$ is the spillover temperature from sources in the side lobes of the telescope, and $T_{\mathrm{RFI}}$ is the increase in system temperature due to terrestrial RFI. The values of $T_{\text {rec }}, T_{\mathrm{CMB}}$, and $T_{\mathrm{gsyn}}$ are well known, but $T_{\text {spill }}$ and $T_{\text {RFI }}$ can vary greatly with time and telescope position causing large uncertainties in $T_{\text {sys }}$ and can only truly be obtained by proper calibration. $T_{\text {spill }}$ is around $5 \mathrm{~K}$ but $T_{\mathrm{RFI}}$ may be a few $\mathrm{K}$ to $100 \mathrm{~s}$ of $\mathrm{K}$. Due to pulsar survey observations rarely ever having accurate calibration, the expression for $T_{\text {sys }}$ has been simplified to include only the $T_{\mathrm{rec}}, T_{\mathrm{CMB}}$, and $T_{\mathrm{gsyn}}$. One last fact to mention is that, due to these pulsars having long periods, DM smearing and scattering are unlikely to be important and are not considered in this work.

For any surveys which were not carried out at $1400 \mathrm{MHz}$, the quoted limits are scaled from the observing frequency to $1400 \mathrm{MHz}$ using a simple power law $S \propto \nu^{-1.6}$, which is consistent with the average spectral behavior for a large sample of normal pulsars (Lorimer et al. 1995).

\subsection{The potentially observable population of young pulsars in GCs}

For all clusters listed in Table 4.3, we can use the flux density limits to model the most likely number of potentially observable pulsars ${ }^{2}$ in each cluster. Using

\footnotetext{
${ }^{2}$ The estimates in this section do not account for beaming effects, or the fact that many pulsars will escape the cluster potential and will be missed by the surveys. We discuss these issues in $\S 4.5$.
} 
a binomial data model, we carry out a simple Bayesian analysis described below. This method rests on a key simplifying assumption about the luminosity function of young pulsars. Following FK06, we will assume that the parent population follows a log-normal luminosity function defined (at $1400 \mathrm{MHz}$ ) to have a mean in the base-10 logarithm of $L$ (in mJy) to be -1.1 and a standard deviation of 0.9 . This assumption is reasonable if we consider that the spin-down evolution of isolated pulsars in GCs is the same as the Galactic disk. Variations on the mean and standard deviation of the log-normal luminosity function have been applied to our analysis using models from Ridley \& Lorimer (2010). These values are in the range of -1.04 to -1.19 for the mean of the luminosity function in the base-10 and in the range of 0.91 to 0.98 for the standard deviation.

We can model the number of pulsars in a particular cluster, $N$, given a "detection probability $\theta$ " and an observed sample of $n$ pulsars. Bayes' theorem gives the joint posterior probability density for $N$ and $\theta$ as

$$
p(N, \theta \mid n) \propto p(n \mid N, \theta) p(N, \theta)
$$

Here $p(n \mid N, \theta)$ is the probability of observing $n$ pulsars from a parent population of $N$ with some $\theta$, and $p(N, \theta)$ is the joint prior probability density for $N$ and $\theta$. The prior distribution for $N$ is assumed to be uniform in the range $n$ to $\infty$. Graphically, $\theta$ is the ratio of the area under the luminosity function for $L>L_{\min }$ to the total area under the function. We evaluate $\theta$ numerically using Monte Carlo integration of the Faucher-Giguère \& Kaspi (2006) luminosity function and four luminosity 
functions from Ridley \& Lorimer (2010). The prior distribution for $\theta$ is assumed to be independent of $N$ and uniform in the range $\theta_{\min }$ to $\theta_{\max }$, which are defined to be the minimum and maximum probabilities from the five distribution functions. The simplest choice for a likelihood function is the binomial distribution, i.e.

$$
p(n \mid N, \theta)=\frac{N !}{n !(N-n) !} \theta^{n}(1-\theta)^{N-n}
$$

where $\theta$ depends on the luminosity limit and the assumed parent luminosity function.

For the majority of cases in which there are no detections in a cluster, $n=0$ and the likelihood term simplifies considerably to

$$
p(n \mid N, \theta)=(1-\theta)^{N}
$$

For the cases of NGC 6440 and NGC 6342, where there is one detection, i.e. $n=1$, we have

$$
p(n \mid N, \theta)=N \theta(1-\theta)^{N-1}
$$

while for the two pulsars in NGC 6624, $n=2$ which leads to

$$
p(n \mid N, \theta)=\frac{N(N-1)}{2} \theta^{2}(1-\theta)^{N-2} .
$$

Having found the joint posterior distribution $p(N, \theta \mid n)$, we then marginalize over $\theta$ to get the posterior distribution for $N$ using the appropriate choice for the likelihood function (i.e. Eq. 4.5, 4.6, or 4.7 depending on the value for $n$ in each case) and give 
the $95 \%$ percentile-based credible intervals for $N$ (i.e. the 0.025 and 0.975 percentiles) as well as the median in Table 4.4. Examples of these discrete probability density functions can be seen in Figure 4.2 for four GCs. The cluster 47 Tuc shows the typical shape of a discrete probability density function for a GC with no young pulsars.
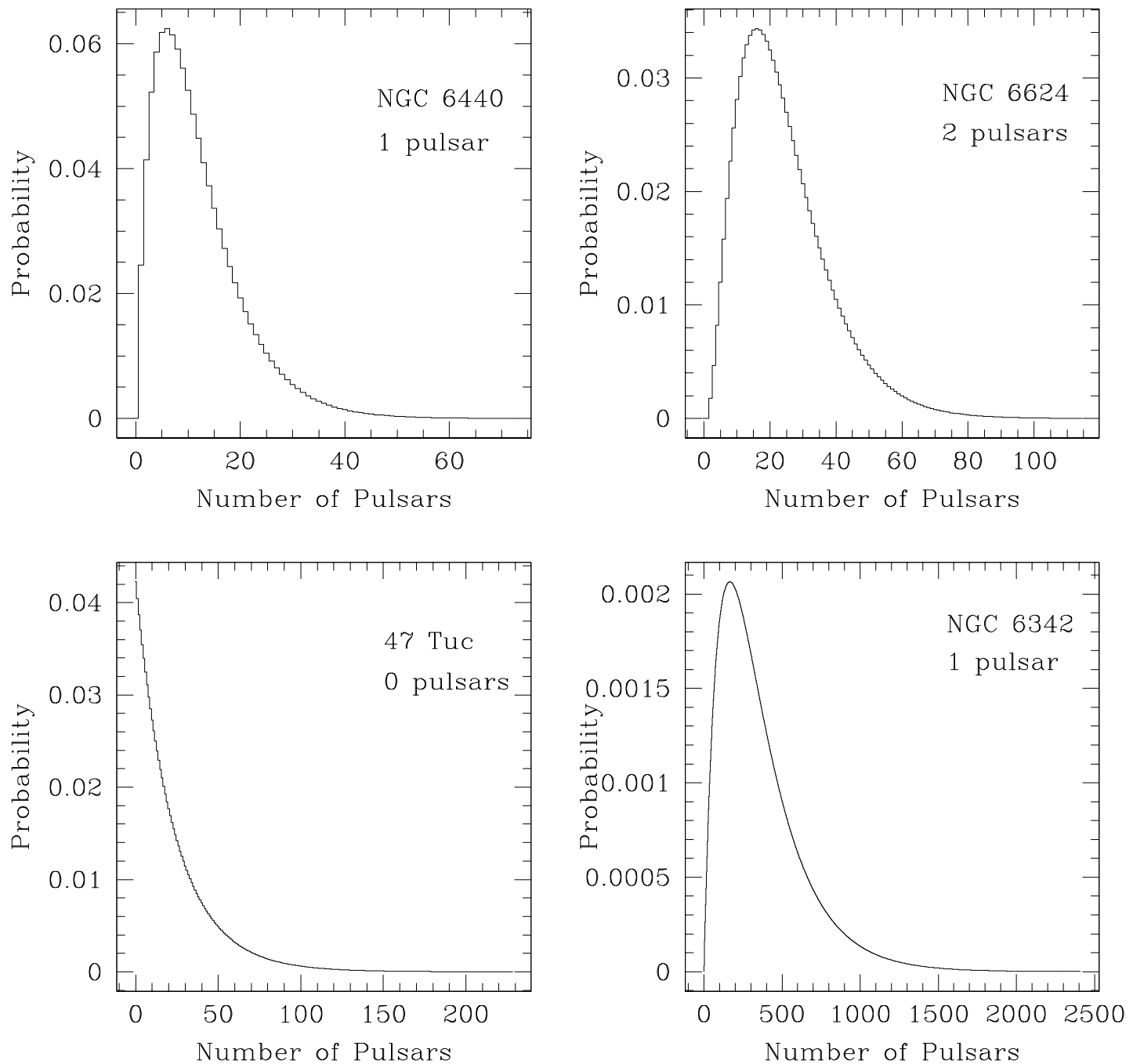

Figure 4.2: Examples of discrete posterior probability density functions for the number of potentially observable pulsars in GCs using the Bayesian analysis. The number of known young pulsars are given in the upper left corner under the GC's name in each plot. 


\subsection{The intrinsic population and birth rates of young GC pulsars}

The results from the previous sections do not take into account the population of young pulsars whose emission beams do not intersect our line of sight or the population of young pulsars which escaped the gravitational potential of their parent GCs. Both of these issues are addressed in section 4.5.1.

\subsubsection{Results for all GCs}

Retention fractions $\left(f_{R}\right)$, the fraction of pulsars which do not have a large enough birth velocity to escape the cluster's gravitational potential, are calculated for each GC. These $f_{R}$ are obtained by using the escape velocity of the GC and a velocity distribution function. Hobbs et al. (2005) showed that a Maxwellian distribution fits the Galactic population of pulsars well for many categories (all, young, recycled, etc.) of pulsars, hence we choose the velocity distribution function to have the form of a Maxwellian with a dispersion $\sigma_{v}$. Caution does need to be taken with this choice of velocity distribution function because no physical mechanism is presented in Hobbs et al. (2005) to explain this Maxwellian distribution and the low velocity end of this distribution is not well constrained due to the fact that low velocity pulsar's proper motions are difficult to measure. Multiple values were chosen for $\sigma_{v}: 265 \mathrm{~km} \mathrm{~s}^{-1}$ from Hobbs et al. (2005), an intermediate value of 130 $\mathrm{km} \mathrm{s}^{-1}, 50 \mathrm{~km} \mathrm{~s}^{-1}, 20 \mathrm{~km} \mathrm{~s}^{-1}$, and $10 \mathrm{~km} \mathrm{~s}^{-1}$ as a lower value. Many separate values for velocity dispersion have been chosen because it is most likely that these young GC pulsars are formed from electron capture supernovae, (see section 4.6.4.2 
for definition), for which the accompanying natal kick may be 10 times smaller than in the case of core collapse supernova (Ivanova et al. 2008, Kitaura et al. 2006). The escape velocities were taken from Gnedin et al. (2002), except for ESO 452-SC11 which came from Webbink (1985). The retention fractions are calculated by numerically integrating the Maxwellian velocity distribution function from 0 to the escape velocity of the GCs.

Each retention fraction was used to calculate a theoretical upper limit for the number of young pulsars produced for every GC

$$
N_{\text {created }}=\frac{N}{f_{R} f_{\text {beam }}}
$$

where $N$ represents the number of pulsars predicted by the binomial method, i.e the median in Table 4.4 , and $f_{\text {beam }}$ is the beaming fraction for pulsars. The value taken for the pulsar beaming fraction is 0.1 (Tauris \& Manchester 1998). This value represents an upper limit on the number of pulsars created in a particular GC. The number of pulsars created in a GC is then divided by the average life time of a young pulsar (43 Myr) to obtain upper limits on the birth rates $(\mathcal{R})$ for each GC. An average lifetime of $43 \mathrm{Myr}$ is derived by taking the total number of simulated Radio-Loud pulsars $(1,200,000)$ and dividing it by the pulsar birthrate $(2.8$ pulsars per century) in FK06. All results from the binomial analysis are contained within Table 4.4 .

One GC, M22, has median value of zero and no young pulsars are expected to be contained within it. This results in a birth rate of zero for M22. To calculate 
a birth rate for this GC, it is assumed that the cluster contains one pulsar and an upper limit is constructed using this assumption.

\subsubsection{Metal-rich GCs}

Up to this point, the analysis presented uses flux density limits and luminosity models as the only considerations for observable pulsars contained within the cluster. Lyne et al. (1996) note that the young pulsars appear in metal-rich GCs and this trend has persisted despite fifteen years of intense searches of most of the cluster population. In this section metallicity will be added as a consideration. For the following discussion Terzan 5 will be excluded due to the possibility that it is not a true GC but a merger of two astrophysical objects that are bound in the Galactic halo (Ferraro et al. 2009). Also excluded will be NGC 6342 due to the uncertainty about PSR B1718-19's membership in the cluster (see $\S 4.2$ ).

The GCs NGC 6440 and NGC 6624 have metallicities that are greater than the $90^{\text {th }}$ percentile of metal rich clusters. These two clusters are two of the three highest metallicity clusters that contain either young or old millisecond pulsars. The probability of selecting two GCs at random in the top three of a ranked list from a sample of 25 GCs is $0.92 \%$. If NGC 6342 is also included in this sample then we have three of the top five highest metallicity clusters with a probability of selecting three of the top five of $0.38 \%$. The inclusion of Terzan 5 (the highest metallicity cluster with any known pulsars) in both of these previous scenarios, changes the probability values from $0.92 \%$ to $1.71 \%$ and $0.38 \%$ to $0.68 \%$. The conjecture can 
be proposed that metal-poor clusters have been selected against for the purpose of surveying GCs due to the belief that metal-rich GCs contain more pulsars overall. Figure 4.3 shows a plot of metallicity versus survey luminosity limit, indicating a random distribution with no bias towards either low or high metallicity clusters.

Figure 4.4 shows the empirical cumulative distribution function (CDF) of the number of predicted young pulsars with the binomial method versus metallicity. GCs with distances greater than $20 \mathrm{kpc}$ are excluded from this CDF because their large distances produce high luminosity limits and in turn create jumps in the CDF. The cause of these jumps seen in Figure 4.4 is due to high survey limits on specific GCs. The first large jump in the CDF at a metallicity of $\sim-0.55$ is due to NGC 6342, the GC with the highest survey luminosity limit.

The GCs with the most known pulsars, Ter 5 and 47 Tuc, have high metallicities and have been used in many simulations. One of these presented in Ivanova et al. (2008) attempts to model the number of young/high magnetic field pulsars in these clusters. In these simulations, three and two young pulsars are predicted to exist in the cores of Ter 5 and 47 Tuc respectively with the most likely creation scenario being the merger of two stars. If beaming and luminosity limits from Table 4.3 are taken into account, the chances of seeing those 2-3 pulsars are extremely small. 


\subsection{Discussion}

\subsubsection{Flux Luminosity Limits}

The results of the binomial analysis depend greatly on the luminosity limits provided from searching these GCs. One can see by comparing the discrete probability density functions of NGC 6342 and NGC 6440 seen in Figure 4.2 that the range of values that each function covers differs greatly. This is a direct result of NGC 6342's flux luminosity limit being much greater than that of NGC 6440. Thus clusters with high flux luminosity limits do not significantly constrain the population/birth rates of these GCs.

Another factor not mentioned is the influence of RFI on searching GCs. The relative impact of RFI on long-period pulsars is much more severe than for millisecond pulsars (MSPs). There is a lot more long-period RFI and the dispersion discrimination between RFI and long-period pulsars is not nearly as great as for MSPs. Therefore this creates a bias against finding such pulsars because they may be ignored assuming they are RFI which is hard to quantify and fully account for.

\subsubsection{Birth Rates in GCs}

For most GCs, the birth rates found are only upper limits with the exception of NGC 6342, NGC 6440, and NGC 6624. Empirical CDFs of these values can be seen in Figure 4.5 for the 97 GCs surveyed. The upper limits on birth rates for velocity dispersions of $265,130,50,20$, and $10 \mathrm{~km} \mathrm{~s}^{-1}$ are $3568,422,24.8,1.67$, and 0.25 pulsars per century respectively. The higher birth rates obtained from the 
higher velocity dispersions are the result of needing to produce more pulsars to get enough pulsars at the low velocity end of the Maxwellian distribution. These values for birth rates provided in this work are much higher than the predicted birth rate of 2.8 pulsars per century for the Galaxy as a whole (FK06). For the purposes of this work, these very high implied birth rates suggest that a very different formation process for young pulsars is occurring in GCs, as well as the possibility that some GCs do not produce young pulsars at all. The major difference in the formation scenario appears to be the lower velocity dispersion at birth.

\subsubsection{Role of cluster metallicity}

In Section 4.5.2, evidence is presented that young pulsars are only present in higher metallicity GCs. Another way to highlight this is to use a simple population model with the GC's mass as the only variable. A pulsar to mass ratio (PMR) is derived using the results of the binomial analysis for NGC 6440 and NGC 6624, each having 10 and 21 pulsars predicted respectively. We do not include the GC NGC 6342 when creating this model because of the uncertain cluster membership of PSR B1718-19 (see §4.2). With NGC 6440's mass of 811,000 $M_{\odot}$ and NGC 6624's mass of $257,000 M_{\odot}$ a PMR of 31 pulsars per 1,068,000 $M_{\odot}$ is obtained. Using the PMR, each of the 97 GCs are revisited and an intrinsic population is predicted for each GC by multiplying the PMR by the GC mass. Using the flux density limits in Table 4.3, the observable population is drawn from the FK06 luminosity model. The results of this simulation can be seen in a histogram in Figure 4.6 and Table 4.4. 
Seventy-four percent of the GCs are predicted to have no observable young pulsars using this model. However, for the remaining GCs, a total of 67 young pulsars should be observable which disagrees with the current population of three by over an order of magnitude. These results show that mass is not a single determining factor in a GC containing young pulsar.

Based on the observed population of young pulsars in the higher metallicity GCs, it may be suggested that lower metallicity GCs may not produce any young pulsars. Figure 4.5 may be used to predict birth rates as a function of metallicity. Given that no young pulsars are observed in GCs with metallicities below -0.6, this value is adopted as a cut-off value. In this case, the population of young pulsars in GCs with $\log [\mathrm{Fe} / \mathrm{H}]>-0.6$ is $447_{-399}^{+1420}$, at $95 \%$ confidence level. This implies an upper limit on the birth rate for GCs with $\log [\mathrm{Fe} / \mathrm{H}]>-0.6$ of $0.012_{-0.010}^{+0.037}$ pulsars per century.

\subsubsection{Formation scenarios}

A few possible situations need to be examined that could explain this current population of normal pulsars in globular clusters.

\subsubsection{Blue Stragglers}

Blue stragglers are the product of the merger of two or possibly three $\sim 1 M_{\odot}$ main sequence stars (Leonard 1989) or binary accretion of a binary companion(s) (McCrea 1964). Stars more massive than $1 M_{\odot}$ have evolved off the main sequence 
many millions of years ago and are not found in GCs. Blue stragglers cannot be the progenitors of the young pulsars because they are not massive enough to result in a core collapse supernova.

\subsubsection{Electron Capture Supernovae}

The creation of these normal pulsars from an electron capture supernova (ECS) of Oxygen-Neon-Magnesium (ONeMg) white dwarfs is another possible avenue. Electron Capture Supernovae occur when a white dwarf gains enough mass to pass its Chandrasekhar mass limit and collapses further. There are three main types of ECSs: accretion-induced collapse (AIC), evolution-induced collapse (EIC), and merger-induced collapse (MIC), of which AIC is the most common (Ivanova et al. 2008). From conservation of magnetic flux, a five order of magnitude change in surface magnetic field is available from an AIC of a white dwarf into a neutron star. This would require a white dwarf to have at least a $10^{7}$ Gauss magnetic field to produce the magnetic field of the young pulsar with the largest magnetic field contained

within a GC. White dwarfs have been observed with magnetic fields above $2 \times 10^{6}$ Gauss and could account for up to $10 \%$ of the white dwarf population (Liebert et al 2003). Therefore, ECSs could be an avenue for the creation of young pulsars. However, Not all white dwarfs that pass the Chandrasekhar limit will collapse via ECS; some will result in type Ia supernova, depending on the composition of the core of the white dwarf.

ECS provide two qualities that are needed to explain the presence of young 
pulsars in GCs. The first is the low velocity dispersion which is needed to keep these young pulsars in their host GCs. The initial formation of the white dwarf results in a small velocity kick of a few $\mathrm{km} / \mathrm{s}$ from either stellar winds or an asymmetric kick during the helium flash (Fregeau et al. 2009). The energetics of the ECS of the white dwarf would produce a small velocity kick (Dessart et al. 2006) and, combined with the velocity kick from previous stages of evolution, would provide a total velocity that is small and would allow most neutron stars created via ECS to be retained by the GC. The second is the higher metallicity of GCs which host young pulsars. An ECS ejects a few $0.001 M_{\odot}$ worth of mass, of which $\sim 25 \%$ is ${ }^{56} \mathrm{Ni}$ (Dessart et al. 2006). ${ }^{56} \mathrm{Ni}$ decays into ${ }^{56} \mathrm{Co}$ with a half-life of $\simeq 6$ days and then ${ }^{56}$ Co decays into ${ }^{56} \mathrm{Fe}$ with a half-life of $\simeq 77$ days. This provides a source of iron creating the higher metallicity for clusters in which all known young pulsars have been detected.

A simple statistical analysis can give an order of magnitude estimate of the possible population of heavy mass $\left(1.0-1.4 M_{\odot}\right)$ white dwarfs available for ECSs. It is assumed that all GCs have the same initial mass function (IMF) and the differences seen between current GC's mass functions are due to the dynamical history of the cluster (Paust et al. 2010). The IMF is a multi-part power-law presented in equation 4 of Kroupa (2002) and with $\alpha_{3}=-2.7$ from Scola (1986). Each GC is assumed to have the same age of 12.4 Gyr for purposes of predicting an initial mass of the GC (Krauss \& Chaboyer 2001). An initial mass is obtained for each GC by using the mass lost fractions presented in Kroupa (2002) for $\alpha_{3}=-2.7$. The number of main sequence stars available for heavy white dwarfs (mass range of $\left.6.0-8.0 M_{\odot}\right)$ are calculated for each GC with a total of $\sim 150,000$ white dwarfs 
formed in these $97 \mathrm{GCs}$. The use of values for $\alpha_{3}$ greater then -2.7 serves only to decrease the numbers of stars in this mass range while choosing the Salpeter index of $\alpha_{3}=-2.35$ only increases the number of stars available by a factor of two (Salpeter 1955). Assuming an interaction age of $10^{9}$ years and that each heavy white dwarf creates one ECS, an occurrence rate of $1.5 \times 10^{-2}$ ECSs per century is obtained and is shown on Figure 4.5 as a solid horizontal black line.

This predicted ECS rate is an order of magnitude less than the birth rates of pulsars for the lowest velocity dispersion. A few factors could bring these values into closer agreement. A decrease in the interaction age would increase the ECS rate. This is a very plausible possibility because there is an accumulation of ECSs at more recent times due to dynamical evolution (it may take a long time for a given capture to form an accreting binary) and individual evolution (it may take several Gyrs to accrete enough mass to cause an ECS). Lower luminosity limits without the detection of more young pulsars would decrease the young pulsar birth rate and bring the two rates closer to agreement. A detailed $N$-body simulation should be used to refine the ECS rate but this is beyond the scope of this work. It is possible that ECSs have only occurred in higher metallicity clusters and not all GCs should be considered in calculating the birthrate of young pulsars. The work presented here suggests that ECS is the most likely creation scenario for young pulsars in GCs. 


\begin{tabular}{lrrrrrrrr}
\hline $\begin{array}{l}\text { Globular } \\
\text { Cluster }\end{array}$ & $\begin{array}{r}l \\
\left(^{o}\right)\end{array}$ & $\begin{array}{c}b \\
\left(^{o}\right)\end{array}$ & $\begin{array}{c}\mathrm{D} \\
(\mathrm{kpc})\end{array}$ & $\begin{array}{c}\mathrm{D}_{\mathrm{GC}} \\
(\mathrm{kpc})\end{array}$ & $\begin{array}{c}r_{t} \\
(\mathrm{pc})\end{array}$ & $\begin{array}{r}S_{\min } \\
\left(\mathrm{mJy}_{\mathrm{pc}}^{2}\right)\end{array}$ & $\begin{array}{r}\text { Mass } \\
\left(M_{\odot}\right)\end{array}$ & $\begin{array}{r}V_{\sigma} \\
\left(\mathrm{km} \mathrm{s}^{-1}\right)\end{array}$ \\
\hline NGC 6325 & 0.9 & 8.0 & 7.8 & 1.1 & 15.8 & 54.8 & 223,000 & 5.9 \\
NGC 6355 & 359.5 & 5.4 & 9.2 & 1.4 & 31.6 & 77.5 & 252,000 & $\mathrm{~N} / \mathrm{A}$ \\
Terzan 2 & 356.3 & 2.3 & 7.5 & 0.8 & 24.0 & none & 3,290 & $\mathrm{~N} / \mathrm{A}$ \\
Terzan 4 & 356.0 & 1.3 & 7.2 & 1.0 & 23.3 & none & $\mathrm{N} / \mathrm{A}$ & $\mathrm{N} / \mathrm{A}$ \\
HP 1 & 357.4 & 2.1 & 8.2 & 0.5 & 20.4 & none & 95,700 & $\mathrm{~N} / \mathrm{A}$ \\
Liller 1 & 354.8 & -0.1 & 8.2 & 0.8 & 36.5 & 9.9 & 289,000 & $\mathrm{~N} / \mathrm{A}$ \\
Terzan 1 & 357.5 & 1.0 & 6.7 & 1.3 & 22.8 & 77.5 & 5,360 & $\mathrm{~N} / \mathrm{A}$ \\
Ton 2 & 350.8 & -3.4 & 8.2 & 1.4 & 25.3 & $n o n e$ & 7,330 & $\mathrm{~N} / \mathrm{A}$ \\
Terzan 5 & 3.8 & 1.6 & 6.9 & 1.2 & 21.9 & 11.6 & 374,000 & $\mathrm{~N} / \mathrm{A}$ \\
NGC 6440 & 7.7 & 3.8 & 8.5 & 1.3 & 13.0 & 9.8 & 811,000 & $\mathrm{~N} / \mathrm{A}$ \\
Terzan 6 & 358.5 & -2.1 & 6.8 & 1.3 & 43.6 & 7.3 & 300,000 & $\mathrm{~N} / \mathrm{A}$ \\
UKS 1 & 5.1 & 0.7 & 7.8 & 0.7 & 45.6 & 54.8 & 145,000 & $\mathrm{~N} / \mathrm{A}$ \\
Terzan 9 & 3.6 & -1.9 & 7.1 & 1.1 & 21.3 & 17.0 & 9,570 & $\mathrm{~N} / \mathrm{A}$ \\
NGC 6522 & 1.0 & -3.9 & 7.7 & 0.6 & 34.8 & 54.8 & 300,000 & 6.7 \\
NGC 6528 & 1.1 & -4.1 & 7.9 & 0.6 & 33.1 & 54.8 & 152,000 & $\mathrm{~N} / \mathrm{A}$ \\
NGC 6558 & 0.2 & -6.0 & 7.4 & 1.0 & 28.5 & 63.3 & 98,400 & 3.1 \\
NGC 6624 & 2.7 & -7.9 & 7.9 & 1.2 & 47.4 & 16.0 & 257,000 & 5.4 \\
\hline
\end{tabular}

Table 4.2: Parameters for Bulge Globular Clusters. $\mathrm{D}_{\mathrm{GC}}$ is the distance from the Galactic center and D is the distance from the Sun to the Globular cluster. GC parameters from Harris (1996)

\subsubsection{Galactic Bulge Pulsars}

One other possibility is that these young pulsars are part of the Galactic field population and are captured by their host GC. The time it would take to travel the distances to the GCs and the time it would take for these pulsars to relax into the core of their host GC have been proposed as evidence against this method. One key fact is neglected here; these are all bulge GCs that contain this population of young pulsars. Their distances from the Galactic center range from 1.2-1.7 kpc and the radius of the Galactic bulge is $\sim 1.5 \mathrm{kpc}$. The time for a pulsar to travel from the outer $0.5 \mathrm{kpc}$ of the Galactic bulge would be short ( $\sim 10^{6}$ years) even for a moderate 
birth velocity kick and the core relaxation time for each GC is an order of magnitude less than the characteristic age of the pulsars that hosts them allowing enough time for them to settle into the core which is where three of the four pulsars are found. The fourth, PSR B1718-19, has a characteristic age about equal to NGC 6342's relaxation time and this could explain why PSR B1718-19 is not located in the host GC's core. This could also explain why these young pulsars are towards the higher end of the age distribution for non-recycled pulsars. The metallicity of the Galactic center is higher than that of the Galactic disk and stars migrating from the bulge to bulge GCs could explain why GCs with young pulsars have higher metallicities compared to the overall GC population.

The population of bulge pulsars is relatively unexplored. Outside of Galactic center surveys (Deneva et al. 2009; Johnston et al. 2006; Klein et al. 2004; \& Kramer et al. 2000) and the surveys of bulge GCs (Table 4.2), the population is unknown. The most sensitive published survey to explore this region is the Parkes Multi-beam survey (Manchester et al. 2001). For pulsars with a period of 100-1000 ms the survey has a limiting flux of $0.16 \mathrm{mJy}$ and this translates into a $L_{\min }$ of $7.8 \mathrm{mJy} \mathrm{kpc}^{2}$ for a distance of $7.0 \mathrm{kpc}$ (front edge of bulge) and $12 \mathrm{mJy} \mathrm{kpc}^{2}$ for a distance of $8.6 \mathrm{kpc}$ (top and bottom of bulge directly above and below Galactic center). These $L_{\min }$ values represent the upper edge of the pulsar luminosity function presented in FK06 and show that the Galactic bulge has not been surveyed well enough to constrain its pulsar population, especially considering that DM smearing and scattering would further hinder the detection of these pulsars.

Another chance for pulsars to be captured will occur when the GC passes 
through the plane of the Galaxy as it orbits the Galactic center. The orbital timescales for globular clusters around the galaxy are hundreds of Myrs and this is longer than the $43 \mathrm{Myr}$ average lifetime of a normal pulsar. This means that any pulsars captured would have to have been captured on the most recent pass of the GC through the Galactic disk. Figure 4.1 shows a small population of pulsars ( $\tau_{\text {char }}>100$ Myrs) that would live long enough to be found in a GC if capture occurred due to this scenario.

The plausibility of star capture by a GC is discussed in Mieske \& Baumgardt (2007; henceforth MB07) and their arguments will be applied here in the following discussions. Table 4.2 lists all GCs within $1.5 \mathrm{kpc}$ of the Galactic center and their characteristics relevant to the MB07 analysis. MB07 found that the capture probability decreases with increasing number of cluster particles $\left(N_{\mathrm{GC}}\right)$ and decreases with increasing initial velocity over cluster velocity dispersion ratio $\left(V_{\text {int }} / \sigma_{\mathrm{GC}}\right)$. Assuming an average particle mass of $0.5 M_{\odot}$, the range of clusters reviewed matches with the range of Galactic bulge clusters, however the range of $V_{\text {int }} / \sigma_{\mathrm{GC}}$ do not. GC velocity dispersions range from $\sim 1.0-19 \mathrm{~km} \mathrm{~s}^{-1}$, which are an order of magnitude less than typical pulsar velocities in previous studies (see Hobbs et al. 2005, for a recent study). The closest scenario of $V_{\mathrm{int}} / \sigma_{\mathrm{GC}}$ equal to unity will be the only one considered from here.

To obtain a comparison of capture rates between pulsars and MB07, a few other considerations need to be put into place. Using the FK06 model for the Galactic pulsar population, the mass density of pulsars is found to be $8.2 \times 10^{-6}$ $M_{\odot} \mathrm{pc}^{-3}$ for the Galactic bulge assuming a uniform pulsar per mass distribution 
throughout the Galaxy. This value is five orders of magnitude less than the value of $0.25 M_{\odot} \mathrm{pc}^{-3}$ used in MB07. Even if the entire pulsar population were placed inside the Galactic bulge, this would increase the mass density by only an order of magnitude. The resulting differences in mass density would produce significantly lower values for the rates found by MB07. The value of $265 \mathrm{~km} \mathrm{~s}^{-1}$ will be adopted as the velocity dispersion $\left(\sigma_{\text {field }}\right)$ for field pulsars (Hobbs et al. 2005). For a $\sigma_{\text {field }}$ of $200 \mathrm{~km} \mathrm{~s}^{-1}$, MB07 concludes that no stars will be captured in any mass cluster within a Hubble time. If the additional constraints for pulsars of lower capture probability due to higher initial velocities and lower capture rates due to lower mass density are included, the conclusion can be drawn that no pulsars are likely to be captured by a GC. Imposing further constraints of beaming, luminosity limits, and finite radio-loud lifetimes for pulsars would further hinder the detection of a pulsar if one were to be captured by a GC. We therefore rule out this possibility as an origin for the young pulsars in GCs.

\subsubsection{Suggested Future Work}

All the work presented here only provides a statistical study at the young pulsar population in GCs and neglects the dynamics and history of each GC. Clearly N-body simulations similar to those presented in Ivanova et al. (2008) of the GCs and their possible interactions with the Galactic stellar and post-stellar populations would place better constraints on the values presented here and would further the understanding of GC's dynamics and evolutionary history. 
To improve upon the observational constraints used in this work, it is clearly desirable to search all GCs as deeply as possible using existing facilities. We highlight here some GCs of particular interest to survey further.

There are a few GCs needing further observations, most notably NGC 6342 which has the highest minimum detectable flux density of any of the 97 GCs and has not to our knowledge been surveyed since the discovery of PSR B1718-19 by Lyne et al. (1993). A new search with the currently available telescopes could reduce the $\mathrm{S}_{\mathrm{min}}$ by a factor of 10 and provide evidence for or against PSR B1718-19 association with NGC 6342 (Bailes et al. 2005, Freire 2005).

Another high metallicity bulge GC to be searched is NGC 6637 (M 69). This cluster, at a distance of $8.8 \mathrm{kpc}$, also has a high two-body encounter rate, $\Gamma$ (Hui et al. 2010). This parameter is often used to assess the plausibility of pulsar content in GCs (see, e.g., Hui et al. 2010).

Most of the GCs presented in this work have been searched to the sensitivity limit of the telescope for which they are visible. The next generation of radio telescopes will be needed to present better constraints on this work. The Square Kilometer Array (SKA) would be an ideal telescope to accomplish this task. The SKA would provide over a factor of one hundred increase in telescope gain over the GBT, Parkes, and Jodrell Bank telescopes and over a factor of thirty in telescope gain over Arecibo. This improvement in gain alone would allow for deep searches, with luminosity limits less than the mean in the FK06 model, in only an hour or two for GCs less than twenty kpc away (see Smits et al. 2009 for a review of pulsar work with the SKA). Before the advent of the SKA, MeerKAT, the South African 
SKA precursor, will be able to reduce these minimum detectable flux density limit by a factor of 10 when completed (Booth et al. 2009). 


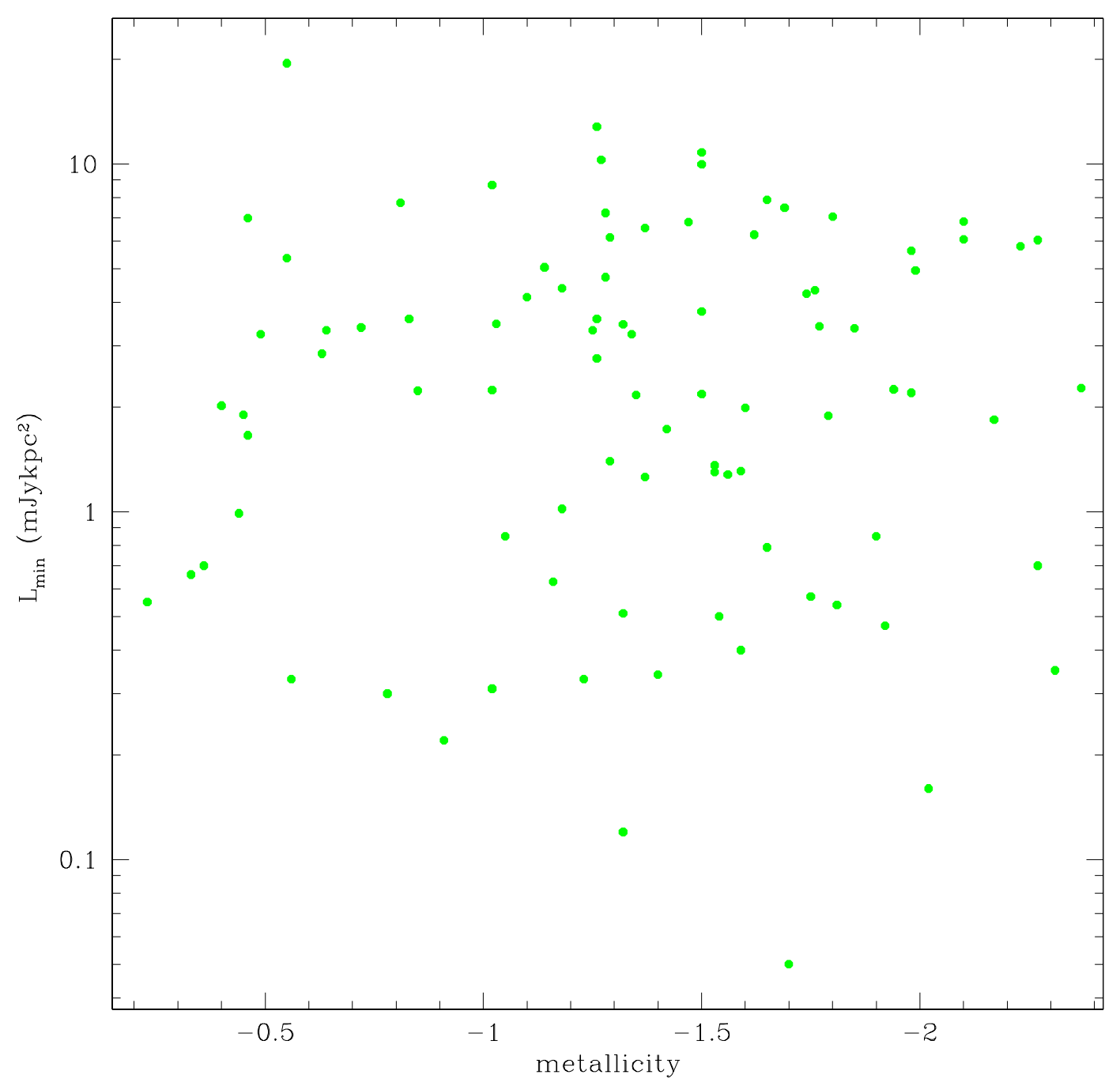

Figure 4.3: Survey luminosity limits at $1400 \mathrm{MHz}$ as a function of metallicity with six GCs excluded with distances greater then $20 \mathrm{kpc}$. The luminosities are randomly distributed and show no bias against low or high metallicity GCs. 


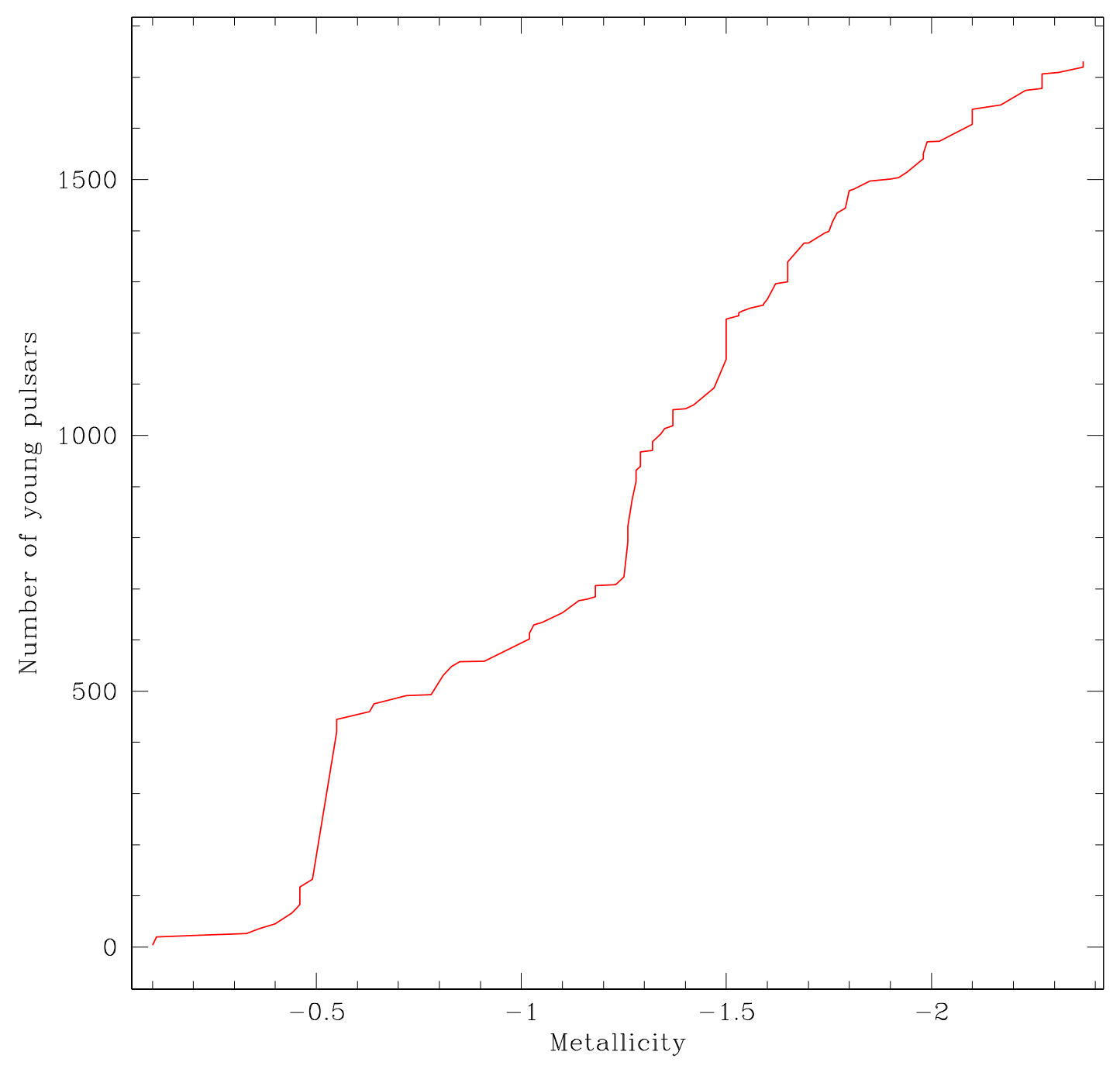

Figure 4.4: CDF of young pulsars predicted by the binomial analysis as a function of metallicity. 


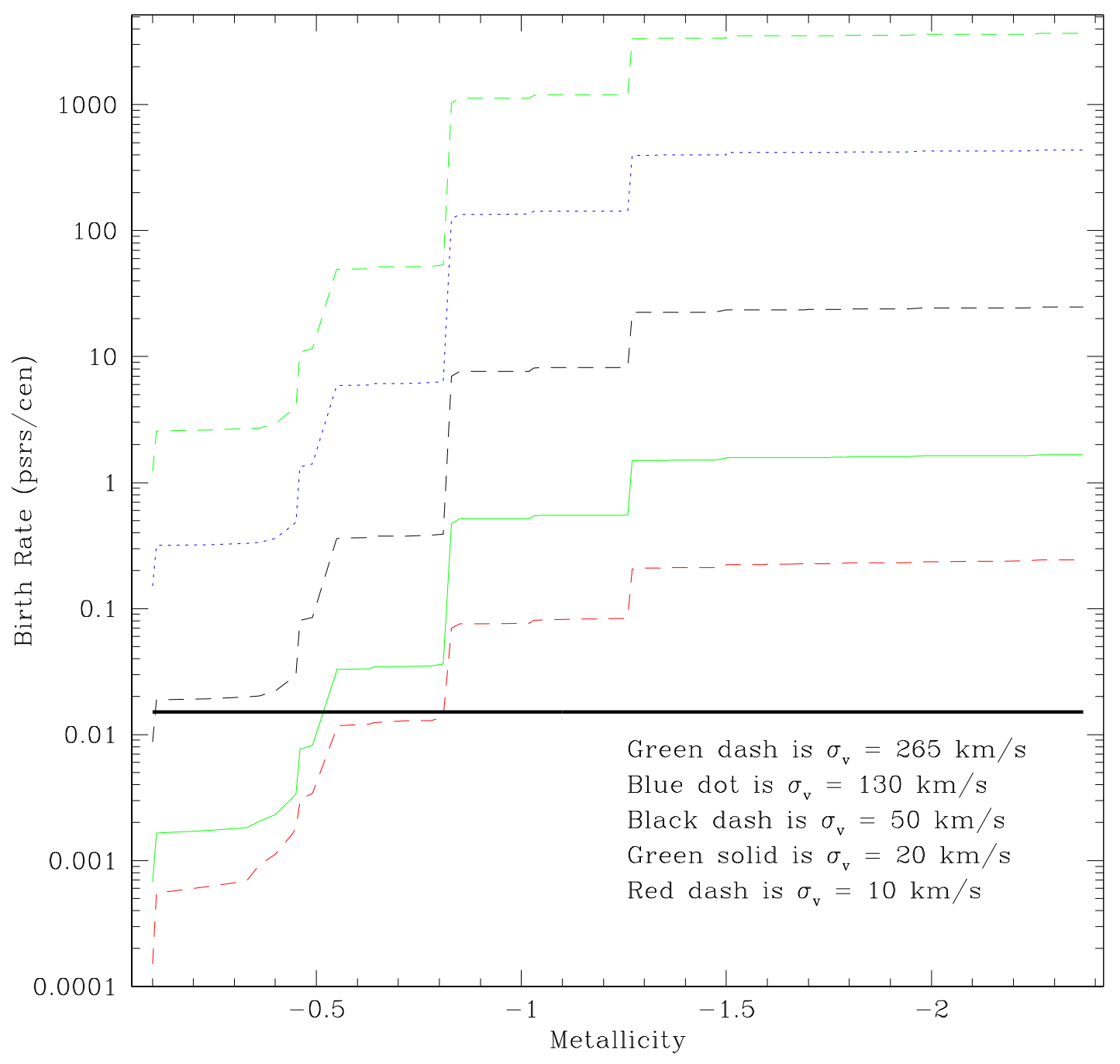

Figure 4.5: CDF of birth rate versus metallicity for multiple velocity dispersions. The solid black line represents an estimate of ECS rates in GCs. 


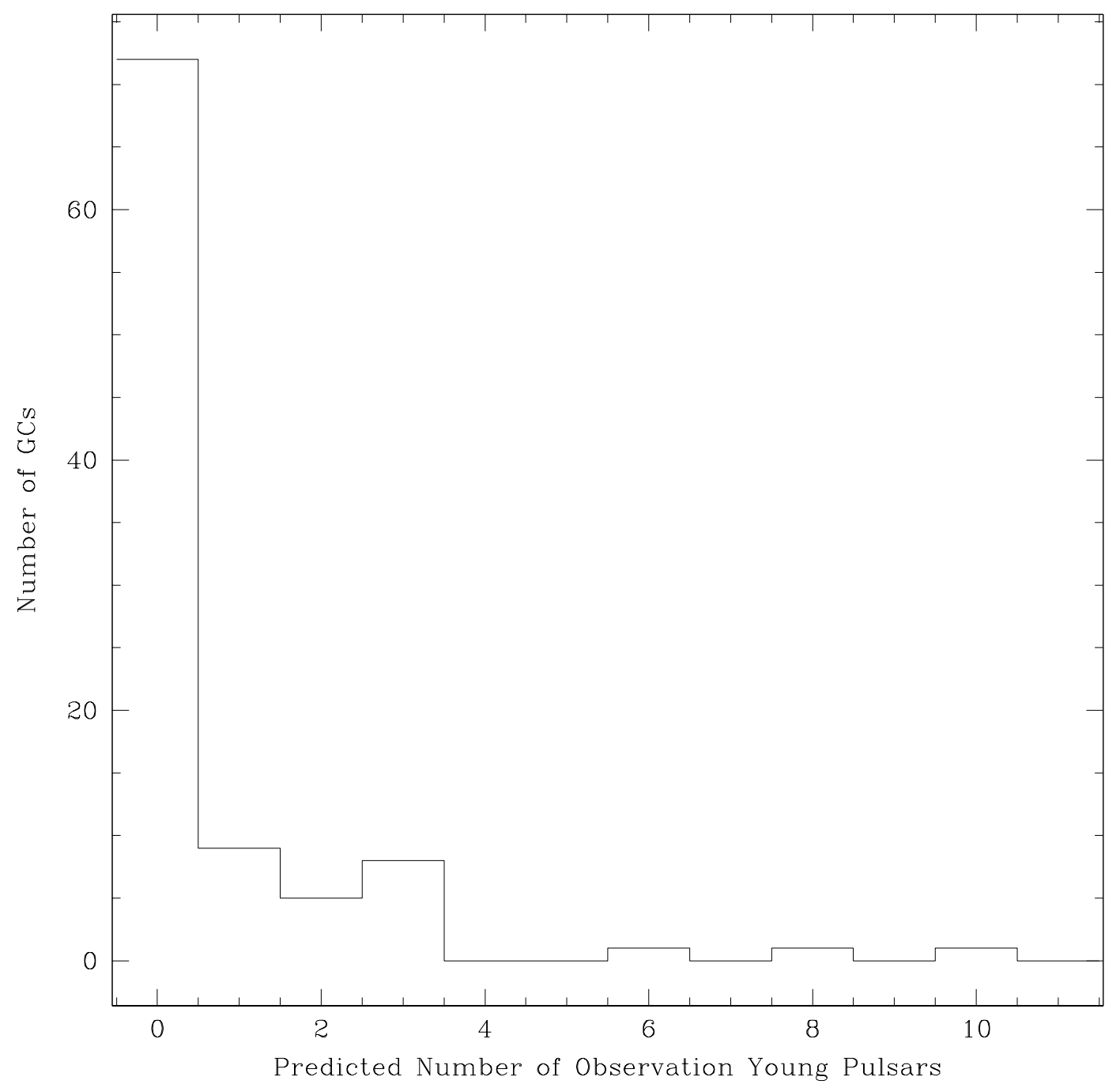

Figure 4.6: Histogram of number of predicted observable young pulsars per GC using a model only dependent on the mass of the GC. 
Table 4.3: Parameters for globular clusters searched for pulsars. GC parameters from Harris (1996).

\begin{tabular}{|c|c|c|c|c|c|c|c|}
\hline $\begin{array}{l}\text { Globular } \\
\text { Cluster }\end{array}$ & $\begin{array}{l}\text { Dist. } \\
\text { (kpc) }\end{array}$ & $\begin{array}{c}S_{\min }(1400) \\
(\mu \mathrm{Jy})\end{array}$ & $\begin{array}{c}V_{\mathrm{esc}} \\
\left(\mathrm{km} \mathrm{s}^{-1}\right)\end{array}$ & $\begin{array}{c}\text { Metallicity } \\
\log [\mathrm{Fe} / \mathrm{H}]\end{array}$ & $\begin{array}{c}\text { Mass } \\
M_{\odot}\end{array}$ & $\begin{array}{l}\text { Number of } \\
\text { Young Pulsars }\end{array}$ & $\begin{array}{c}\text { Search } \\
\text { Reference }\end{array}$ \\
\hline 47 Tuc & 4.5 & 167.6 & 68.8 & -0.72 & $1,500,000$ & 0 & Freire et al. 2001 \\
\hline NGC 288 & 8.9 & 6.5 & 13.3 & -1.32 & 112,000 & 0 & Lynch \& Ransom 2011 \\
\hline NGC 1261 & 16.3 & 38.8 & 3.4 & -1.27 & 341,000 & 0 & Possenti unpublished 2010 \\
\hline Pal 2 & 27.2 & 34 & 27.8 & -1.42 & 410,000 & 0 & Hessels et al. 2007 \\
\hline NGC 1851 & 12.1 & 30.1 & 51.8 & -1.18 & 551,000 & 0 & Freire et al. 2004 \\
\hline NGC 2298 & 10.8 & 4.1 & 18.4 & -1.92 & 84,900 & 0 & Lynch \& Ransom 2011 \\
\hline NGC 2808 & 9.6 & 54.8 & 72.8 & -1.14 & $1,420,000$ & 0 & Possenti unpublished 2010 \\
\hline E3 & 8.1 & 54.8 & 3.0 & -0.83 & 3,290 & 0 & Possenti unpublished 2010 \\
\hline NGC 3201 & 4.9 & 54.8 & 22.0 & -1.59 & 254,000 & 0 & Possenti unpublished 2010 \\
\hline NGC 4147 & 19.3 & 19 & 18.3 & -1.80 & 74,700 & 0 & Hessels et al. 2007 \\
\hline NGC 4372 & 5.8 & 54.8 & 21.5 & -2.17 & 329,000 & 0 & Possenti unpublished 2010 \\
\hline NGC 4590 & 10.3 & 54.8 & 18.2 & -2.23 & 223,000 & 0 & Possenti unpublished 2010 \\
\hline NGC 4833 & 6.6 & 77.5 & 31.8 & -1.85 & 410,000 & 0 & Possenti unpublished 2010 \\
\hline NGC 5024 & 17.9 & 19 & 33.4 & -2.10 & 826,000 & 0 & Hessels et al. 2007 \\
\hline NGC 5053 & 17.4 & 20 & 8.9 & -2.27 & 125,000 & 0 & Hessels et al. 2007 \\
\hline NGC 5139 & 5.2 & 48.3 & 60.4 & -1.53 & $3,350,000$ & 0 & Possenti unpublished 2010 \\
\hline NGC 5272 & 10.2 & 21 & 37.2 & -1.50 & 957,000 & 0 & Hessels et al. 2007 \\
\hline NGC 5286 & 11.7 & 54.8 & 52.6 & -1.69 & 713,000 & 0 & Possenti unpublished 2010 \\
\hline NGC 5466 & 16.0 & 22 & 9.5 & -1.98 & 179,000 & 0 & Hessels et al. 2007 \\
\hline Pal 5 & 23.2 & 32 & 3.2 & -1.41 & 30,000 & 0 & Hessels et al. 2007 \\
\hline NGC 5897 & 12.5 & 5.5 & 13.4 & -1.90 & 211,000 & 0 & Lynch \& Ransom 2011 \\
\hline NGC 5904 & 7.5 & 25 & 47.7 & -1.29 & 857,000 & 0 & Hessels et al. 2007 \\
\hline NGC 5927 & 7.7 & 54.8 & 33.9 & -0.49 & 338,000 & 0 & Possenti unpublished 2010 \\
\hline NGC 5946 & 10.6 & 54.8 & 25.3 & -1.29 & 281,000 & 0 & Possenti unpublished 2010 \\
\hline NGC 5986 & 10.4 & 3.7 & 37.0 & -1.59 & 599,000 & 0 & Lynch \& Ransom 2011 \\
\hline M 80 & 10.0 & 5.7 & 48.7 & -1.75 & 502,000 & 0 & Lynch et al. 2011 \\
\hline NGC 6121 & 2.2 & 131.4 & 34.2 & -1.16 & 195,000 & 0 & Lyne et al. 1988 \\
\hline $\mathrm{ESO} 452$ & 8.3 & 54.8 & 5.9 & -1.50 & 75,000 & 0 & Possenti unpublished 2010 \\
\hline NGC 6144 & 8.9 & 54.8 & 14.1 & -1.76 & 169,000 & 0 & Possenti unpublished 2010 \\
\hline NGC 6139 & 10.1 & 77.5 & 59 & -1.65 & 566,000 & 0 & Possenti unpublished 2010 \\
\hline NGC 6171 & 6.4 & 54.8 & 25 & -1.02 & 182,000 & 0 & Possenti unpublished 2010 \\
\hline NGC 6205 & 7.1 & 27 & 39.1 & -1.53 & 775,000 & 0 & Hessels et al. 2007 \\
\hline NGC 6218 & 4.8 & 54.8 & 28.5 & -1.37 & 217,000 & 0 & Possenti unpublished 2010 \\
\hline NGC 6235 & 11.5 & 54.8 & 16.8 & -1.28 & 73,300 & 0 & Possenti unpublished 2010 \\
\hline NGC 6254 & 4.4 & 66.6 & 29.5 & -1.56 & 252,000 & 0 & Possenti unpublished 2010 \\
\hline Pal 15 & 45.1 & 38 & 4.3 & -2.07 & 40,300 & 0 & Hessels et al. 2007 \\
\hline NGC 6266 & 6.8 & 22.1 & 97.8 & -1.18 & $1,220,000$ & 0 & Chandler 2003 \\
\hline NGC 6273 & 8.8 & 54.8 & 58.4 & -1.74 & $1,100,000$ & 0 & Possenti unpublished 2010 \\
\hline NGC 6284 & 15.3 & 54.8 & 28.6 & -1.26 & 361,000 & 0 & Possenti unpublished 2010 \\
\hline NGC 6287 & 9.4 & 77.5 & 30.4 & -2.10 & 188,000 & 0 & Possenti unpublished 2010 \\
\hline NGC 6293 & 9.5 & 54.8 & 41.7 & -1.99 & 329,000 & 0 & Possenti unpublished 2010 \\
\hline NGC 6304 & 5.9 & 54.8 & 38.4 & -0.45 & 217,000 & 0 & Possenti unpublished 2010 \\
\hline M 92 & 8.3 & 5.1 & 47.1 & -2.31 & 489,000 & 0 & Lynch \& Ransom 2011 \\
\hline NGC 6325 & 7.8 & 54.8 & 42.5 & -1.25 & 223,000 & 0 & Possenti unpublished 2010 \\
\hline NGC 6333 & 7.9 & 54.8 & 37.7 & -1.77 & 422,000 & 0 & Possenti unpublished 2010 \\
\hline NGC 6342 & 8.5 & 270 & 22.9 & -0.55 & 96,600 & 1 & Biggs \& Lyne 1996 \\
\hline NGC 6355 & 9.2 & 77.5 & 40.3 & -1.37 & 252,000 & 0 & Possenti unpublished 2010 \\
\hline Liller 1 & 8.2 & 9.9 & 41.2 & -0.33 & 289,000 & 0 & Lynch et al. 2011 \\
\hline Ter 1 & 6.7 & 77.5 & 7.4 & -1.03 & 5,360 & 0 & Possenti unpublished 2010 \\
\hline NGC 6388 & 9.9 & 54.8 & 124 & -0.55 & $2,170,000$ & 0 & Possenti unpublished 2010 \\
\hline NGC 6402 & 9.3 & 54.8 & 39.1 & -1.28 & $1,040,000$ & 0 & Possenti unpublished 2010 \\
\hline NGC 6401 & 10.6 & 77.5 & 38.3 & -1.02 & 286,000 & 0 & Possenti unpublished 2010 \\
\hline NGC 6397 & 2.3 & 31.5 & 48.3 & -2.02 & 115,000 & 0 & Possenti unpublished 2010 \\
\hline Pal 6 & 5.8 & 6.7 & 28.0 & -0.91 & 228,000 & 0 & Lynch et al. 2011 \\
\hline NGC 6426 & 20.6 & 25 & 14.7 & -2.15 & 117,000 & 0 & Hessels et al. 2007 \\
\hline Ter 5 & 6.9 & 11.6 & 50.5 & -0.23 & 374,000 & 0 & Ransom et al. 2005 \\
\hline NGC 6440 & 8.5 & 9.8 & 85.2 & -0.36 & 811,000 & 1 & Freire et al. 2008 \\
\hline NGC 6441 & 11.6 & 12.4 & 102 & -0.46 & $1,570,000$ & 0 & Freire et al. 2008 \\
\hline Ter 6 & 6.8 & 7.3 & 38.3 & -0.56 & 300,000 & 0 & Lynch et al. 2011 \\
\hline NGC 6453 & 11.6 & 80.4 & 22.4 & -1.50 & 169,000 & 0 & Possenti unpublished 2010 \\
\hline UKS 1 & 7.8 & 54.8 & 25.4 & -0.64 & 145,000 & 0 & Possenti unpublished 2010 \\
\hline NGC 6496 & 11.3 & 54.8 & 19.7 & -0.46 & 200,000 & 0 & Possenti unpublished 2010 \\
\hline Ter 9 & 7.1 & 17.0 & 9.8 & -1.05 & 9,570 & 0 & Lynch \& Ransom 2011 \\
\hline NGC 6517 & 10.6 & 3.0 & 82.9 & -1.23 & 526,000 & 0 & Lynch et al. 2011 \\
\hline NGC 6522 & 7.7 & 54.8 & 42.3 & -1.34 & 300,000 & 0 & Possenti unpublished 2010 \\
\hline NGC 6528 & 7.9 & 54.8 & 26.4 & -0.11 & 152,000 & 0 & Possenti unpublished 2010 \\
\hline NGC 6535 & 6.8 & 41 & 10.0 & -1.79 & 20,000 & 0 & Hessels et al. 2007 \\
\hline NGC 6539 & 7.8 & 47.0 & 35.8 & -0.63 & 536,000 & 0 & D'Amico et al. 1993 \\
\hline
\end{tabular}


Table 4.3: Continued

\begin{tabular}{|c|c|c|c|c|c|c|c|}
\hline $\begin{array}{l}\text { Globular } \\
\text { Cluster }\end{array}$ & $\begin{array}{l}\text { Dist. } \\
\text { (kpc) }\end{array}$ & $\begin{array}{c}S_{\min }(1400) \\
(\mu \mathrm{Jy})\end{array}$ & $\begin{array}{c}V_{\mathrm{esc}} \\
\left(\mathrm{km} \mathrm{s}^{-1}\right)\end{array}$ & $\begin{array}{l}\text { Metallicity } \\
\log [\mathrm{Fe} / \mathrm{H}]\end{array}$ & $\begin{array}{c}\text { Mass } \\
M_{\odot}\end{array}$ & $\begin{array}{l}\text { Number of } \\
\text { Young Pulsars }\end{array}$ & $\begin{array}{c}\text { Search } \\
\text { Reference }\end{array}$ \\
\hline NGC 6540 & 5.3 & 77.5 & 27.6 & -1.35 & 36,400 & 0 & Possenti unpublished 2010 \\
\hline NGC 6544 & 3.0 & 38.8 & 93.5 & -1.40 & 108,000 & 0 & Possenti unpublished 2010 \\
\hline NGC 6541 & 7.5 & 9.6 & 42.2 & -1.81 & 572,000 & 0 & Lynch unpublished 2010 \\
\hline NGC 6558 & 7.4 & 63.3 & 32.1 & -1.32 & 98,400 & 0 & Possenti unpublished 2010 \\
\hline NGC 6584 & 13.5 & 54.8 & 24.3 & -1.50 & 303,000 & 0 & Possenti unpublished 2010 \\
\hline NGC 6624 & 7.9 & 16.0 & 35.3 & -0.44 & 257,000 & 2 & Lynch et al. 2011 \\
\hline M 28 & 5.5 & 4.1 & 63.8 & -1.32 & 551,000 & 0 & Lynch unpublished 2010 \\
\hline NGC 6642 & 8.1 & 54.8 & 30.7 & -1.26 & 109,000 & 0 & Possenti unpublished 2010 \\
\hline NGC 6652 & 10.0 & 77.5 & 37.5 & -0.81 & 109,000 & 0 & Possenti unpublished 2010 \\
\hline M 22 & 3.2 & 5.7 & 44.7 & -1.70 & 644,000 & 0 & Lynch et al. 2011 \\
\hline NGC 6681 & 9.0 & 77.5 & 39.3 & -1.62 & 179,000 & 0 & Possenti unpublished 2010 \\
\hline NGC 6712 & 6.9 & 6.7 & 27.7 & -1.02 & 257,000 & 0 & Lynch et al. 2011 \\
\hline NGC 6717 & 7.1 & 54.8 & 21.6 & -1.26 & 47,500 & 0 & Possenti unpublished 2010 \\
\hline NGC 6723 & 8.7 & 54.8 & 27.3 & -1.10 & 357,000 & 0 & Possenti unpublished 2010 \\
\hline NGC 6749 & 7.9 & 32 & 20.4 & -1.60 & 123,000 & 0 & Hessels et al. 2007 \\
\hline NGC 6752 & 4.0 & 31.5 & 32.9 & -1.54 & 317,000 & 0 & Possenti unpublished 2010 \\
\hline NGC 6760 & 7.4 & 37 & 40.1 & -0.40 & 357,000 & 0 & Hessels et al. 2007 \\
\hline NGC 6779 & 9.4 & 25 & 28.7 & -1.98 & 230,000 & 0 & Hessels et al. 2007 \\
\hline Pal 10 & 5.9 & 23 & 17.2 & -0.10 & 53,100 & 0 & Hessels et al. 2007 \\
\hline NGC 6809 & 5.4 & 77.5 & 19.7 & -1.94 & 269,000 & 0 & Possenti unpublished 2010 \\
\hline NGC 6838 & 4.0 & 19 & 16.7 & -0.78 & 43,000 & 0 & Hessels et al. 2007 \\
\hline NGC 6934 & 15.6 & 28 & 28.1 & -1.47 & 295,000 & 0 & Hessels et al. 2007 \\
\hline NGC 6981 & 17.0 & 6.0 & 16.6 & -1.42 & 168,000 & 0 & Lynch \& Ransom 2011 \\
\hline NGC 7006 & 41.2 & 19 & 19.8 & -1.52 & 303,000 & 0 & Hessels et al. 2007 \\
\hline NGC 7078 & 10.4 & 21 & 62.1 & -2.37 & $1,190,000$ & 0 & Hessels et al. 2007 \\
\hline NGC 7089 & 11.5 & 6.0 & 48.1 & -1.65 & 104,000 & 0 & Lynch \& Ransom 2011 \\
\hline NGC 7099 & 8.1 & 10.8 & 34.1 & -2.27 & 241,000 & 0 & Ransom et al. 2004 \\
\hline Pal 12 & 19.0 & 6.2 & 5.5 & -0.85 & 15,900 & 0 & Lynch \& Ransom 2011 \\
\hline Pal 13 & 26.0 & 21 & 3.5 & -1.88 & 6,500 & 0 & Hessels et al. 2007 \\
\hline
\end{tabular}


Table 4.4: Binomial analysis of young pulsars in globular clusters.

\begin{tabular}{|c|c|c|c|c|c|c|c|c|}
\hline $\begin{array}{l}\text { Globular } \\
\text { Cluster }\end{array}$ & Median & $\begin{array}{c}2.5^{\mathrm{th}} \\
\text { percentile }\end{array}$ & $\begin{array}{c}97.5^{\text {th }} \\
\text { percentile }\end{array}$ & $\begin{array}{c}\log \left(f_{R 1}\right) \\
50 \mathrm{~km} \mathrm{~s}^{-1}\end{array}$ & $\begin{array}{c}\log \left(f_{R 3}\right) \\
10 \mathrm{~km} \mathrm{~s}^{-1}\end{array}$ & $\begin{array}{c}\log (\mathcal{R}) f_{R 1} \\
\left(\operatorname{psrs~cen~}^{-1}\right)\end{array}$ & $\begin{array}{c}\log (\mathcal{R}) f_{R 3} \\
\left(\text { psrs cen }^{-1}\right)\end{array}$ & $\begin{array}{l}\mathrm{N}_{\text {predicted }} \\
\text { mass model }\end{array}$ \\
\hline 47 Tuc & 16 & 0 & 88 & -0.39 & 0.00 & -3.04 & -3.43 & 1 \\
\hline NGC 288 & 3 & 0 & 17 & -2.30 & -0.42 & -1.85 & -3.74 & 0 \\
\hline NGC 1261 & 53 & 1 & 297 & -4.08 & -1.99 & 1.15 & -0.92 & 0 \\
\hline Pal 2 & 167 & 6 & 968 & -1.37 & -0.02 & -1.03 & -2.40 & 0 \\
\hline NGC 1851 & 21 & 0 & 115 & -0.66 & 0.00 & -2.65 & -3.32 & 0 \\
\hline NGC 2298 & 3 & 0 & 16 & -1.89 & -0.17 & -2.26 & -3.99 & 3 \\
\hline NGC 2808 & 24 & 0 & 133 & -0.34 & 0.00 & -2.92 & -3.26 & 0 \\
\hline E3 & 17 & 0 & 93 & -4.24 & -2.15 & 0.82 & -1.25 & 0 \\
\hline NGC 3201 & 6 & 0 & 36 & -1.66 & -0.08 & -2.19 & -3.77 & 0 \\
\hline NGC 4147 & 34 & 1 & 192 & -1.90 & -0.17 & -1.20 & -2.93 & 0 \\
\hline NGC 4372 & 9 & 0 & 49 & -1.70 & -0.09 & -1.99 & -3.59 & 0 \\
\hline NGC 4590 & 28 & 1 & 155 & -1.90 & -0.18 & -1.28 & -3.01 & 0 \\
\hline NGC 4833 & 16 & 0 & 87 & -1.21 & 0.00 & -2.22 & -3.43 & 0 \\
\hline NGC 5024 & 29 & 1 & 163 & -1.15 & 0.00 & -2.02 & -3.17 & 0 \\
\hline NGC 5053 & 29 & 1 & 162 & -2.83 & -0.82 & -0.35 & -2.35 & 0 \\
\hline NGC 5139 & 6 & 0 & 36 & -0.50 & 0.00 & -3.35 & -3.86 & 8 \\
\hline NGC 5272 & 10 & 0 & 57 & -1.03 & 0.00 & -2.61 & -3.64 & 1 \\
\hline NGC 5286 & 37 & 1 & 205 & -0.64 & 0.00 & -2.42 & -3.07 & 0 \\
\hline NGC 5466 & 27 & 0 & 149 & -2.74 & -0.75 & -0.46 & -2.45 & 0 \\
\hline Pal 5 & 101 & 3 & 575 & -4.16 & -2.07 & 1.51 & -0.56 & 0 \\
\hline NGC 5897 & 4 & 0 & 25 & -2.30 & -0.41 & -1.73 & -3.62 & 0 \\
\hline NGC 5904 & 7 & 0 & 39 & -0.75 & 0.00 & -3.04 & -3.80 & 2 \\
\hline NGC 5927 & 15 & 0 & 84 & -1.13 & 0.00 & -2.32 & -3.46 & 0 \\
\hline NGC 5946 & 29 & 1 & 164 & -1.49 & -0.03 & -1.68 & -3.13 & 0 \\
\hline NGC 5986 & 2 & 0 & 14 & -1.03 & 0.00 & -3.30 & -4.34 & 3 \\
\hline M 80 & 3 & 0 & 19 & -0.73 & 0.00 & -3.43 & -4.16 & 6 \\
\hline NGC 6121 & 3 & 0 & 20 & -1.13 & 0.00 & -3.03 & -4.16 & 0 \\
\hline ESO452 & 17 & 0 & 98 & -3.36 & -1.30 & -0.04 & -2.10 & 0 \\
\hline NGC 6144 & 20 & 0 & 113 & -2.23 & -0.36 & -1.10 & -2.97 & 0 \\
\hline NGC 6139 & 39 & 1 & 218 & -0.53 & 0.00 & -2.51 & -3.05 & 0 \\
\hline NGC 6171 & 10 & 0 & 59 & -1.50 & -0.04 & -2.13 & -3.60 & 0 \\
\hline NGC 6205 & 7 & 0 & 37 & -0.97 & 0.00 & -2.82 & -3.80 & 1 \\
\hline NGC 6218 & 6 & 0 & 35 & -1.34 & -0.01 & -2.51 & -3.84 & 0 \\
\hline NGC 6235 & 35 & 1 & 197 & -2.01 & -0.23 & -1.08 & -2.86 & 0 \\
\hline NGC 6254 & 6 & 0 & 36 & -1.30 & -0.01 & -2.55 & -3.85 & 0 \\
\hline Pal 15 & 878 & 30 & 5409 & -3.77 & -1.70 & 2.07 & 0.00 & 0 \\
\hline NGC 6266 & 5 & 0 & 29 & -0.13 & 0.00 & -3.80 & -3.94 & 3 \\
\hline NGC 6273 & 20 & 0 & 110 & -0.54 & 0.00 & -2.80 & -3.34 & 0 \\
\hline NGC 6284 & 69 & 2 & 391 & -1.34 & -0.01 & -1.46 & -2.78 & 0 \\
\hline NGC 6287 & 33 & 1 & 185 & -1.26 & 0.00 & -1.85 & -3.11 & 0 \\
\hline NGC 6293 & 23 & 0 & 130 & -0.90 & 0.00 & -2.38 & -3.28 & 0 \\
\hline NGC 6304 & 9 & 0 & 51 & -0.99 & 0.00 & -2.69 & -3.69 & 0 \\
\hline M 92 & 2 & 0 & 13 & -0.76 & 0.00 & -3.57 & -4.34 & 2 \\
\hline NGC 6325 & 15 & 0 & 86 & -0.87 & 0.00 & -2.58 & -3.46 & 0 \\
\hline NGC 6333 & 16 & 0 & 88 & -1.01 & 0.00 & -2.42 & -3.43 & 0 \\
\hline NGC 6342 & 288 & 40 & 1047 & -1.61 & -0.06 & -0.56 & -2.11 & 0 \\
\hline NGC 6355 & 31 & 1 & 176 & -0.93 & 0.00 & -2.21 & -3.15 & 0 \\
\hline Liller 1 & 3 & 0 & 21 & -0.91 & 0.00 & -3.25 & -4.16 & 0 \\
\hline Ter 1 & 16 & 0 & 90 & -3.06 & -1.03 & -0.36 & -2.40 & 0 \\
\hline NGC 6388 & 25 & 0 & 142 & -0.04 & 0.00 & -3.20 & -3.24 & 1 \\
\hline NGC 6402 & 22 & 0 & 124 & -0.97 & 0.00 & -2.32 & -3.30 & 0 \\
\hline NGC 6401 & 43 & 1 & 244 & -0.99 & 0.00 & -2.01 & -3.00 & 0 \\
\hline NGC 6397 & 1 & 0 & 8 & -0.73 & 0.00 & -3.90 & -4.64 & 1 \\
\hline Pal 6 & 1 & 0 & 10 & -1.36 & -0.02 & -3.27 & -4.62 & 2 \\
\hline NGC 6426 & 55 & 1 & 308 & -2.18 & -0.33 & -0.72 & -2.56 & 0 \\
\hline Ter 5 & 3 & 0 & 18 & -0.69 & 0.00 & -3.47 & -4.16 & 2 \\
\hline NGC 6440 & 10 & 2 & 34 & -0.22 & 0.00 & -3.41 & -3.64 & 3 \\
\hline NGC 6441 & 8 & 0 & 45 & -0.12 & 0.00 & -3.62 & -3.74 & 3 \\
\hline Ter 6 & 2 & 0 & 13 & -0.99 & 0.00 & -3.34 & -4.34 & 1 \\
\hline NGC 6453 & 56 & 2 & 316 & -1.64 & -0.07 & -1.24 & -2.81 & 0 \\
\hline UKS 1 & 15 & 0 & 86 & -1.49 & -0.03 & -1.97 & -3.42 & 0 \\
\hline NGC 6496 & 34 & 1 & 189 & -1.80 & -0.13 & -1.30 & -2.97 & 0 \\
\hline Ter 9 & 4 & 0 & 25 & -2.70 & -0.72 & -1.33 & -3.32 & 0 \\
\hline NGC 6517 & 2 & 0 & 13 & -0.24 & 0.00 & -4.10 & -4.34 & 3 \\
\hline NGC 6522 & 15 & 0 & 84 & -0.88 & 0.00 & -2.58 & -3.46 & 0 \\
\hline NGC 6528 & 16 & 0 & 88 & -1.44 & -0.03 & -1.99 & -3.40 & 0 \\
\hline NGC 6535 & 9 & 0 & 50 & -2.67 & -0.70 & -1.01 & -2.98 & 0 \\
\hline NGC 6539 & 13 & 0 & 74 & -1.07 & 0.00 & -2.45 & -3.53 & 0 \\
\hline NGC 6540 & 10 & 0 & 57 & -1.38 & -0.02 & -2.25 & -3.62 & 0 \\
\hline NGC 6544 & 2 & 0 & 13 & -0.16 & 0.00 & -4.17 & -4.34 & 0 \\
\hline NGC 6541 & 3 & 0 & 18 & -0.88 & 0.00 & -3.28 & -4.16 & 3 \\
\hline NGC 6558 & 16 & 0 & 90 & -1.20 & 0.00 & -2.23 & -3.43 & 0 \\
\hline NGC 6584 & 51 & 1 & 287 & -1.54 & -0.05 & -1.39 & -2.88 & 0 \\
\hline NGC 6624 & 21 & 5 & 58 & -1.09 & 0.00 & -2.23 & -3.32 & 0 \\
\hline M28 & 1 & 0 & 7 & -0.45 & 0.00 & -4.18 & -4.64 & 10 \\
\hline NGC 6642 & 17 & 0 & 93 & -1.25 & 0.00 & -2.15 & -3.40 & 0 \\
\hline NGC 6652 & 38 & 1 & 213 & -1.02 & 0.00 & -2.04 & -3.06 & 0 \\
\hline M 22 & 0 & 0 & 4 & -0.82 & 0.00 & $-1.74^{a}$ & $-4.64^{a}$ & 0 \\
\hline NGC 6681 & 30 & 1 & 168 & -0.96 & 0.00 & $\begin{array}{l}1.14 \\
-2.20\end{array}$ & $\begin{array}{l}-3.04 \\
-3.16\end{array}$ & 0 \\
\hline NGC 6712 & 2 & 0 & 12 & -1.38 & -0.02 & -2.96 & -4.32 & 1 \\
\hline NGC 6717 & 13 & 0 & 72 & -1.69 & -0.09 & -1.83 & -3.43 & 0 \\
\hline NGC 6723 & 19 & 0 & 108 & -1.40 & -0.02 & -1.96 & -3.33 & 0 \\
\hline NGC 6749 & 9 & 0 & 53 & -1.76 & -0.12 & -1.92 & -3.56 & 0 \\
\hline NGC 6752 & 3 & 0 & 17 & -1.17 & 0.00 & -2.99 & -4.16 & 1 \\
\hline NGC 6760 & 9 & 0 & 53 & -0.94 & 0.00 & -2.74 & -3.69 & 0 \\
\hline NGC 6779 & 10 & 0 & 58 & -1.33 & -0.01 & -2.30 & -3.62 & 0 \\
\hline Pal 10 & 4 & 0 & 24 & -1.98 & -0.21 & -2.06 & -3.82 & 0 \\
\hline
\end{tabular}


Table 4.4: Continued

\begin{tabular}{|c|c|c|c|c|c|c|c|c|}
\hline $\begin{array}{l}\text { Globular } \\
\text { Cluster }\end{array}$ & Median & $\begin{array}{c}2.5^{\text {th }} \\
\text { percentile }\end{array}$ & $\begin{array}{c}97.5^{\text {th }} \\
\text { percentile }\end{array}$ & $\begin{array}{c}\log \left(f_{R 1}\right) \\
50 \mathrm{~km} \mathrm{~s}^{-1}\end{array}$ & $\begin{array}{c}\log \left(f_{R 3}\right) \\
10 \mathrm{~km} \mathrm{~s}^{-1}\end{array}$ & $\begin{array}{c}\log (\mathcal{R}) f_{R 1} \\
\left(\operatorname{psrs~cen~}^{-1}\right)\end{array}$ & $\begin{array}{c}\log (\mathcal{R}) f_{R 3} \\
\left(\text { psrs cen }^{-1}\right)\end{array}$ & $\begin{array}{l}\mathrm{N}_{\text {predicted }} \\
\text { mass model }\end{array}$ \\
\hline NGC 6809 & 10 & 0 & 59 & -1.80 & -0.13 & -1.83 & -3.50 & 0 \\
\hline NGC 6838 & 2 & 0 & 12 & -2.01 & -0.23 & -2.32 & -4.10 & 0 \\
\hline NGC 6934 & 33 & 1 & 184 & -1.36 & -0.01 & -1.75 & -3.10 & 0 \\
\hline NGC 6981 & 8 & 0 & 46 & -2.02 & -0.24 & -1.71 & -3.49 & 0 \\
\hline NGC 7006 & 237 & 8 & 1384 & -1.80 & -0.13 & -0.46 & -2.13 & 0 \\
\hline NGC 7078 & 11 & 0 & 60 & -0.48 & 0.00 & -3.11 & $\begin{array}{l}-2.10 \\
-3.60\end{array}$ & 2 \\
\hline NGC 7089 & 4 & 0 & 24 & -0.74 & 0.00 & -3.30 & -4.04 & 3 \\
\hline NGC 7099 & 4 & 0 & 22 & -1.13 & 0.00 & -2.90 & -4.03 & 1 \\
\hline Pal 12 & 10 & 0 & 59 & -3.45 & -1.39 & -0.18 & -2.25 & 0 \\
\hline Pal 13 & 79 & 2 & 446 & -4.04 & -1.96 & 1.29 & -0.78 & 0 \\
\hline
\end{tabular}

${ }^{a}$ Values are upper limits assuming M22 contains one pulsar. $f_{R}$ is the retention fraction for a given velocity dispersion.

$\mathcal{R}$ is the birthrate in pulsars per century for the median value. Birthrates are upper limits for a given globular cluster except for those with detected young pulsars. 


\section{Chapter 5}

\section{Conclusions and Future Work}

\subsection{Green Bank 350-MHz surveys}

In Chapter 2 we explored the pulsar population at a radio frequency of 350MHz. So far this survey has resulted in the discovery of twenty-seven new pulsars and many redetected pulsars up to a DM of $307 \mathrm{pc} \mathrm{cm}^{-3}$. Among these 27, there are six binary systems, six millisecond pulsars, two partially recycled pulsars, and one rotating radio transient. The periods of these new sources range from 1.7 milliseconds to 5.0 seconds covering a wide range of pulsar periods, but due to interstellar medium effects, the DM range only covers 3.27 to $66.98 \mathrm{pc} \mathrm{cm}^{-3}$. The discoveries from this survey have led to a wealth of multi-wavelength observations

In the high energy regime, two of these sources, PSR J1023+0038 and PSR J2256-1022 were detected with the Fermi Gamma-ray satellite and both show pulsations. At least three sources, PSR J1023+0038, PSR J2256-1022, and PSR J2222-0137, have X-ray detected counterparts and PSR J1023+0038 shows pulsations in the X-ray. Optical companions have been detected for PSR J1023+0038 and PSR J0348+0432 but none has been found for PSR J2222-0137, though it is the closest pulsar found in this survey. VLBA could provide distances to many of these sources, but only sources of interest have existing VLBA observation conducted on them. This includes PSR J1023+0038 with a distance of $1.6 \mathrm{kpc}$ and PSR 
J2222-0137 with a distance of 250 pc. Masses of two pulsars, PSR J1023+0038 with a mass of $1.67 M_{\odot}$ and PSR J2222-0137 with a mass of $1.49 M_{\odot}$, have been measured, using different techniques.

The optical limit of 24.5 mags and the current VLBA parallax measurement suggests that PSR J2222-0137 and its companion are a double neutron star system. If confirmed, this would make it the closest, least eccentric double neutron system known, with an eccentricity two orders of magnitude lower than any previously measured. Searches across many radio frequencies have been undertaken with no detection of a pulsar as the companion. With a probability of detection of $98 \%$ using the luminosity model from FK06, the companion is most likely beaming away from our line of sight.

The GBT 350-MHz drift-scan pulsar survey was a pilot for the new Green Bank North Celestrial Cap (GBNCC) survey. The GBNCC takes advantage of the newest Green Bank pulsar instrument called the Green Bank Ultimate Pulsar Instrument (GUPPI; Ransom et al 2009). The survey will cover all the sky visible from Green Bank and so far has found more than eleven new pulsars including one, PSR J0645+51, already added to existing pulsar timing arrays.

Such a large survey will allow the luminosity function for pulsars at low frequencies to be explored. Similar modeling has been done with the PMPS and is widely used for examining the pulsar population (Faucher-Giguère \& Kaspi 2006; Ridley \& Lorimer 2010). These works are conducted at $1400 \mathrm{MHz}$ and require scaling to be used for population modeling at other frequencies. Such scaling may provide incorrect numbers of pulsars due to the exclusion of steep spectral index pulsars 
that may be only observable at lower frequencies and pulsars with gigahertz-peaked spectral turnovers (Kijak et al 2011). Although a number of low-frequency pulsar surveys have been carried out over the years, the non-uniform sensitivity makes it difficult to model this effect. The GBNCC will be an excellent survey to explore the frequency evolution of the pulsar luminosity function at lower frequencies.

\subsection{Rotating Radio Transients}

In Chapter 3 we took a look at a small population of the rotating radio transients. Here we obtained five timing solutions and looked at the properties of each rotating radio transient to determine how they fit into the population of neutron stars. Some of these sources are like normal pulsars, just their emissions shows a greater range of single pulse fluxes than what is seen in most pulsars. Others are truly erratic and the pulsar model needs to explain the exotic behavior such as pulsars that spend most of their time not emitting in the radio.

So far only about thirty percent of the known RRATs have timing solutions. Further work needs to be done to obtain more solutions in order to characterize the population. Along with this, full Stokes observations should occur to measure other properties of the RRATs' emission. Lastly, more examples of this sporadically emitting population are needed and will be found by current and ongoing surveys. 


\subsection{Globular Cluster Pulsars}

In Chapter 4 we looked at the unusual population of long period pulsars in Galactic globular clusters. Three globular clusters contain four of these objects. Using Bayesian statistics and luminosity limits, we inferred a possible population of long period pulsars and constructed a possible birthrate to examine sources for this population. The collapse of an $\mathrm{O}-\mathrm{Ne}-\mathrm{Mg}$ white dwarf was determined to be the most likely creation mechanism for these neutron stars. Also noted was that these pulsars were hosted by globular clusters that had high iron metallicity, possibly providing a link between core collapse supernovae and the metallicity of the host globular clusters.

Most globular clusters have been searched to the sensitivity limit available for telescopes that can observe them. A few exceptions exist like NGC 6342 that have very large flux density limits and could easily be improved with the newest generation of instruments. Along with clusters not searched that deeply, there are a few like NGC 6637 that have favorable cluster properties and may contain pulsars both normal and recycled. Further theoretical work also needs to be done to better understand core collapse supernovae and the effects they could have on the stellar environment of globular clusters.

\subsection{Next Generation Radio Telescopes}

All the projects undertaken in this work would benefit from the next generation of telescopes. In the long term the Square Kilometer Array (SKA) will be the 
ultimate instrument for all radio studies in $0.5-10 \mathrm{GHz}$ frequency bands. The SKA is only in the design stage, so the next telescopes will be SKA precursors like MeerKAT or other new interferometers like LOFAR will allow for greater sensitivity than the current generation of telescopes. 


\section{Bibliography}

Anderson, P. W., \& Itoh, N. 1975, Nature, 256, 25

Anderson, 1992 Ph.D. Thesis, Cal Tech

Archibald, A. M., et al. 2009, Science, 324, 1411

Baade, W., \& Zwicky, F. 1934, Proceedings of the National Academy of Science, 20, 254

Backer, D. C., Kulkarni, S. R., Heiles, C., Davis, M. M., \& Goss, W. M. 1982, Nature, 300, 615

Backer, D. C., Foster, R. S., \& Sallmen, S. 1993, Nature, 365, 817

Bailes, M., Zhu, J., \& Richter, S. 2005, Binary Radio Pulsars, 328, 195

Bhat, N. D. R., Cordes, J. M., Camilo, F., Nice, D. J., \& Lorimer, D. R. 2004, ApJ, 605,759

Biggs, J. D., \& Lyne, A. G. 1996, MNRAS, 282, 691

Booth, R. S., de Blok, W. J. G., Jonas, J. L., \& Fanaroff, B. 2009, arXiv:0910.2935

Briley, M. M., Bell, R. A., Hesser, J. E., \& Smith, G. H. 1994, Canadian Journal of Physics, 72, 772

Camilo, F., Lorimer, D. R., Freire, P., Lyne, A. G., \& Manchester, R. N. 2000, ApJ, 535,975

Camilo, F., et al. 2001, ApJ, 548, L187

Camilo, F., \& Rasio, F. A. 2005, Binary Radio Pulsars, 328, 147

Champion, D. J., Ransom, S. M., Lazarus, P., et al. 2008, Science, 320, 1309

Chandler, A. 2003 Ph.D. Thesis, Cal Tech

Cordes, J. M., \& Lazio, T. J. W. 2002, arXiv:astro-ph/0207156

Cordes, J. M., Freire, P. C. C., Lorimer, D. R., et al. 2006, ApJ, 637, 446

Cordes, J. M., \& Shannon, R. M. 2008, ApJ, 682, 1152

D'Amico, N., Bailes, M., Lyne, A. G., Manchester, R. N., Johnston, S., Fruchter, A. S., \& Goss, W. M. 1993, MNRAS, 260, L7

Damashek, M., Taylor, J. H., \& Hulse, R. A. 1978, ApJ, 225, L31

Deller, A. T., Tingay, S. J., Bailes, M., \& Reynolds, J. E. 2009, ApJ, 701, 1243 
Deneva, J. S., Cordes, J. M., \& Lazio, T. J. W. 2009, ApJ, 702, L177

Dessart, L., Burrows, A., Ott, C. D., Livne, E., Yoon, S.-C., \& Langer, N. 2006, ApJ, 644, 1063

Dewey, R. J., Taylor, J. H., Weisberg, J. M., \& Stokes, G. H. 1985, ApJ, 294, L25

Eatough, R. P., Molkenthin, N., Kramer, M., Noutsos, A., Keith, M. J., Stappers, B. W., \& Lyne, A. G. 2010, MNRAS, 407, 2443

Faucher-Giguère, C.-A., \& Kaspi, V. M. 2006, ApJ, 643, 332

Ferraro, F. R., et al. 2009, Nature, 462, 483

Foster, R. S., \& Backer, D. C. 1990, ApJ, 361, 300

Fregeau, J. M., Richer, H. B., Rasio, F. A., \& Hurley, J. R. 2009, ApJ, 695, L20

Freire, P. C., Camilo, F., Lorimer, D. R., Lyne, A. G., Manchester, R. N., \& D'Amico, N. 2001, MNRAS, 326, 901

Freire, P. C., Gupta, Y., Ransom, S. M., \& Ishwara-Chandra, C. H. 2004, ApJ, 606, L53

Freire, P. C. C. 2005, Binary Radio Pulsars, 328, 405

Freire, P. C. C., Ransom, S. M., Bégin, S., Stairs, I. H., Hessels, J. W. T., Frey, L. H., \& Camilo, F. 2008, ApJ, 675, 670

Freire, P. C. C., Bassa, C. G., Wex, N., et al. 2011, MNRAS, 412, 2763

Gnedin, O. Y., Zhao, H., Pringle, J. E., Fall, S. M., Livio, M., \& Meylan, G. 2002, ApJ, 568, L23

Gold, T. 1969, Nature, 221, 25

Hankins, T. H., Kern, J. S., Weatherall, J. C., \& Eilek, J. A. 2003, Nature, 422, 141

Harris, W. E. 1996, AJ, 112, 1487

Hessels, J. W. T., Ransom, S. M., Stairs, I. H., et al. 2006, Science, 311, 1901

Hessels, J. W. T., Ransom, S. M., Stairs, I. H., Kaspi, V. M., \& Freire, P. C. C. 2007, ApJ, 670, 363

Hessels, J. W. T., Ransom, S. M., Kaspi, V. M., Roberts, M. S. E., Champion, D. J., \& Stappers, B. W. 2008, 40 Years of Pulsars: Millisecond Pulsars, Magnetars and More, 983, 613

Hewish, A., Bell, S. J., Pilkington, J. D. H., Scott, P. F., \& Collins, R. A. 1968, Nature, 217, 709 
Hobbs, G., Lorimer, D. R., Lyne, A. G., \& Kramer, M. 2005, MNRAS, 360, 974

Hobbs, G., et al. 2010, Classical and Quantum Gravity, 27, 084013

Hotan, A. W., van Straten, W., \& Manchester, R. N. 2004, PASA, 21, 302

Hui, C. Y., Cheng, K. S., \& Taam, R. E. 2010, ApJ, 714, 1149

Jacoby, B. A., Cameron, P. B., Jenet, F. A., Anderson, S. B., Murty, R. N., \& Kulkarni, S. R. 2006, ApJ, 644, L113

Johnston, S., Kramer, M., Lorimer, D. R., Lyne, A. G., McLaughlin, M., Klein, B., \& Manchester, R. N. 2006, MNRAS, 373, L6

Kaplan, D. L., et al. 2005, PASP, 117, 643

Keane, E. F., Kramer, M., Lyne, A. G., Stappers, B. W., \& McLaughlin, M. A. 2011, MNRAS, 415, 3065

Kijak, J., Lewandowski, W., Maron, O., Gupta, Y., \& Jessner, A. 2011, Astron. Astrophys., 531, A16

Kitaura, F. S., Janka, H.-T., \& Hillebrandt, W. 2006, Astron. Astrophys., 450, 345

Klein, B., Kramer, M., Müller, P., \& Wielebinski, R. 2004, Young Neutron Stars and Their Environments, 218, 133

Kramer, M., Klein, B., Lorimer, D., Müller, P., Jessner, A., \& Wielebinski, R. 2000, IAU Colloq. 177: Pulsar Astronomy - 2000 and Beyond, 202, 37

Kramer, M., Lyne, A. G., Hobbs, G., et al. 2003, ApJ, 593, L31

Kramer, M., Stairs, I. H., Manchester, R. N., et al. 2006, Science, 314, 97

Krauss, L. M., \& Chaboyer, B. 2001, arXiv:astro-ph/0111597

Kroupa, P. 2002, Science, 295, 82

Kurkela, A., Romatschke, P., \& Vuorinen, A. 2010, Physics Review D, 81, 105021

Lattimer, J. M., \& Prakash, M. 2001, ApJ, 550, 426

Leonard, P. J. T. 1989, AJ, 98, 217

Li, X.-D. 2006, ApJ, 646, L139

Liebert, J., Bergeron, P., \& Holberg, J. B. 2003, AJ, 125, 348

Lorimer, D. R., Yates, J. A., Lyne, A. G., \& Gould, D. M. 1995, MNRAS, 273, 411

Lorimer, D. R. \& Kramer, M. 2005 Handbook of Pulsar Astronomy 
Luo, Q., \& Melrose, D. 2007, MNRAS, 378, 1481

Lynch, R. S., \& Ransom, S. M. 2011, arXiv:1101.0763

Lynch, R. S., Ransom, S. M., Freire, P. C. C., \& Stairs, I. H. 2011, ApJ, 734, 89

Lynch, R. S., Ransom, S. M., Freire, P. 2011, in preparation

Lynch, R. S. 2011 in preparation

Lyne, A. G., Brinklow, A., Middleditch, J., Kulkarni, S. R., \& Backer, D. C. 1987, Nature, 328, 399

Lyne, A. G., Biggs, J. D., Brinklow, A., McKenna, J., \& Ashworth, M. 1988, Nature, 332,45

Lyne, A. G., Biggs, J. D., Harrison, P. A., \& Bailes, M. 1993, Nature, 361, 47

Lyne, A. G., Manchester, R. N., \& D’Amico, N. 1996, ApJ, 460, L41

Lyne, A. G., et al. 2004, Science, 303, 1153

Manchester, R. N., Lyne, A. G., D’Amico, N., et al. 1996, MNRAS, 279, 1235

Manchester, R. N., et al. 2001, MNRAS, 328, 17

Manchester, R. N., Hobbs, G. B., Teoh, A., \& Hobbs, M. 2005, VizieR Online Data Catalog, 7245, 0

McCrea, W. H. 1964, MNRAS, 128, 147

McLaughlin, M. A., et al. 2006, Nature, 439, 817

McLaughlin, M. A., Lyne, A. G., Keane, E. F., et al. 2009, MNRAS, 400, 1431

Mieske, S., \& Baumgardt, H. 2007, Astron. Astrophys., 475, 851

Morris, D. J., et al. 2002, MNRAS, 335, 275

Paust, N. E. Q., et al. 2010, AJ, 139, 476

Phinney, E. S. 1992, Royal Society of London Philosophical Transactions Series A, 341,39

Phinney, E. S. 1993, Pulsars as Physics Laboratories, 39

Possenti, A., et al. 2011, unpublished

Radhakrishnan, V., \& Cooke, D. J. 1969, ApJ letters, 3, 225

Ransom, S. M. 2001, Ph.D.thesis, Harvard University

Ransom, S. M., Eikenberry, S. S., \& Middleditch, J. 2002, AJ, 124, 1788 
Ransom, S. M., Stairs, I. H., Backer, D. C., Greenhill, L. J., Bassa, C. G., Hessels, J. W. T., \& Kaspi, V. M. 2004, ApJ, 604, 328

Ransom, S. M., Hessels, J. W. T., Stairs, I. H., Freire, P. C. C., Camilo, F., Kaspi, V. M., \& Kaplan, D. L. 2005, Science, 307, 892

Ransom, S. M., Demorest, P., Ford, J., McCullough, R., Ray, J., DuPlain, R., \& Brandt, P. 2009, American Astronomical Society Meeting Abstracts, 214, \#605.08

Ridley, J. P., \& Lorimer, D. R. 2010, MNRAS, 404, 1081

Rosen, R., Heatherly, S., McLaughlin, M. A., et al. 2010, Astronomy Education Review, 9, 010106

Salpeter, E. E. 1955, ApJ, 121, 161

Sayer, R. W., Nice, D. J., \& Taylor, J. H. 1997, ApJ, 474, 426

Scalo, J. M. 1986, Fund. Cosmic Phys., 11, 1

Shapiro, I. I. 1964, Physical Review Letters, 13, 789

Shklovskii, I. S. 1970, Sov. Astron., 13, 562

Sieber, W. 1973, Astron. Astrophys., 28, 237

Smits, R., Kramer, M., Stappers, B., Lorimer, D. R., Cordes, J., \& Faulkner, A. 2009, Astron. Astrophys., 493, 1161

Srinivasan, G. 1989, A\&A Rev., 1, 209

Stairs, I. H., Manchester, R. N., Lyne, A. G., et al. 2001, MNRAS, 325, 979

Stokes, G. H., Taylor, J. H., Welsberg, J. M., \& Dewey, R. J. 1985, Nature, 317, 787

Tauris, T. M., \& Manchester, R. N. 1998, MNRAS, 298, 625

Taylor, J. H., \& Huguenin, G. R. 1969, Nature, 221, 816

Taylor, J. H., Wolszczan, A., Damour, T., \& Weisberg, J. M. 1992, Nature, 355, 132

Taylor, J. H. 1992, Philosophical Transactions of the Royal Society of London, 341, 117-134 (1992), 341, 117

Taylor, J. H., \& Cordes, J. M. 1993, ApJ, 411, 674

Webbink, R. F. 1985, Dynamics of Star Clusters, 113, 541

Weltevrede, P., Stappers, B. W., Rankin, J. M., \& Wright, G. A. E. 2006, ApJ, 645, L149 
Wolszczan, A., Kulkarni, S. R., Middleditch, J., Backer, D. C., Fruchter, A. S., \& Dewey, R. J. 1989, Nature, 337, 531

Wolszczan, A., \& Frail, D. A. 1992, Nature, 355, 145

Yan, W. M., et al. 2011, MNRAS, 414, 2087 